\title{
WestVirginiaUniversity
}

THE RESEARCH REPOSITORY @ WVU

Graduate Theses, Dissertations, and Problem Reports

2003

\section{Spline based controller for nonlinear systems}

Ali Karimi

West Virginia University

Follow this and additional works at: https://researchrepository.wvu.edu/etd

\section{Recommended Citation}

Karimi, Ali, "Spline based controller for nonlinear systems" (2003). Graduate Theses, Dissertations, and Problem Reports. 1414.

https://researchrepository.wvu.edu/etd/1414

This Thesis is protected by copyright and/or related rights. It has been brought to you by the The Research Repository @ WVU with permission from the rights-holder(s). You are free to use this Thesis in any way that is permitted by the copyright and related rights legislation that applies to your use. For other uses you must obtain permission from the rights-holder(s) directly, unless additional rights are indicated by a Creative Commons license in the record and/ or on the work itself. This Thesis has been accepted for inclusion in WVU Graduate Theses, Dissertations, and Problem Reports collection by an authorized administrator of The Research Repository @ WVU. For more information, please contact researchrepository@mail.wvu.edu. 


\title{
Spline Based Controller For Nonlinear Systems
}

\author{
by \\ Ali Karimi \\ Thesis submitted to the \\ College of Engineering and Mineral Resources \\ at West Virginia University \\ in partial fulfillment of the requirements \\ for the degree of \\ Master of Science \\ in \\ Electrical Engineering \\ Professor Asadollah Davari, Ph.D. \\ Professor Powsiri Klinkhachorn, Ph.D. \\ Professor Sherman D. Riemenschneider, Ph.D. \\ Professor Ali Feliachi, Ph.D., Chair
}

Lane Department of Computer Science and Electrical Engineering

Morgantown, West Virginia

2003

Keywords: spline, transient stability, nonlinear control, internal variable identifier

Copyright 2003 Ali Karimi 


\author{
Abstract \\ Spline Based Controller For Nonlinear Systems \\ by \\ Ali Karimi \\ Master of Science in Electrical Engineering \\ West Virginia University \\ Professor Ali Feliachi, Ph.D., Chair
}

The objective of this thesis is to apply spline theory to implement controllers for nonlinear systems. Two different systems, forced duffing oscillators and power systems, are investigated. The spline method is used to mimic the controller which drives a state of the Duffing system toward a desired path. The spline-based nonlinear controller has piecewise polynomial segments with different order of polynomials on each segment. Controller efforts for different order of polynomial interpolants and power spectral densities of the controller signals are compared with the exact feedback linearizaton method.

The first objective for power systems is to design nonlinear excitation controllers for a multimachine power system using Direct Feedback Linearization. The designed controllers, whose parameters are obtained, require the internal variables of the machines. These variables are verified by using a proposed internal variable identifying algorithm. The objective is to design nonlinear excitation controllers for power system stability enhancement. Spline techniques are used to approximate the nonlinear controllers obtained through feedback linearization by piecewise polynomials while enhancing the stability of the system. 


\section{Acknowledgments}

I would like to take this opportunity to express my sincere and deep appreciation to Ali Feliachi, Ph.D., my advisor and mentor. His wisdom and expertise have been invaluable to me since I began my studies in the engineering program at WVU, and I would not have been able to complete this project without his guidance. Special thanks also go to Sherman Riemenschneider, Ph.D., Assadollah Davari, Ph.D., Powsiri Klinkhachorn, Ph.D. and Karl E. Schoder, Ph.D. for their expert assistance and support throughout this project.

It is also important to note that I could not have succeeded without the support of my parents, Kamran and Afarin Karimi, and my brother, Malek Khosrow Karimi. Even though they live thousands of miles away, I have felt their sustaining love and encouragement every day over the past two years. Special thanks go to my aunt, Noushin Nemazee, her husband, Reza, and their sons, Shahin and Ali. They have given me a home away from home, as well as strong support and love. Next, I would like to express my appreciation to my cousin, Aidin Vaziri, and his wife Carol for being there for me. 


\section{Contents}

Acknowledgments iii

List of Figures vi vi vis

List of Tables $\quad$ viii

$\begin{array}{lll}1 & \text { Introduction } & 1\end{array}$

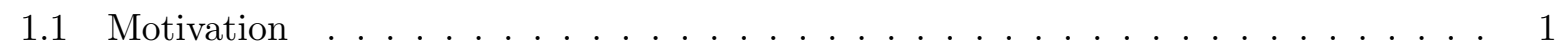

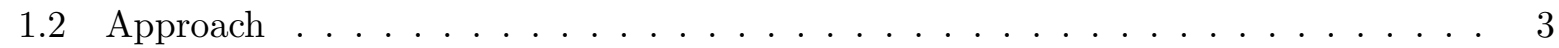

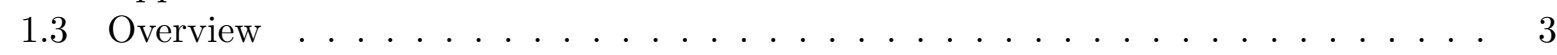

\begin{tabular}{|ll|l}
2 & Literature Survey & 4
\end{tabular}

2.1 Nonlinear Oscillators . . . . . . . . . . . . . . . . . . . . . . . . . . . 4

2.1 .1 Definitions . . . . . . . . . . . . . . . . . . . 4

2.1 .2 Mathematical Model . . . . . . . . . . . . . . . . . . . . . 7

$2.1 .3 \quad$ Problem Description . . . . . . . . . . . . . . . . . . . . . . . . . . 9

2.1 .4 Controller Design . . . . . . . . . . . . . . . . . . . . . . . . . . . 10

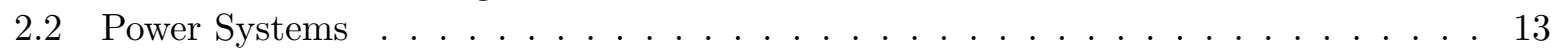

2.2 .1 Power System Dynamics . . . . . . . . . . . . . . . . . . . . . . . . . . . . . . . . . . . . . . .

2.2 .2 Stability Enhancement . . . . . . . . . . . . . . . . . . . . 14

2.2 .3 Problem Description $\ldots \ldots \ldots \ldots$. . . . . . . . . . . . . . . . . 15

$\begin{array}{llr}3 & \text { Spline Theory } & 18\end{array}$

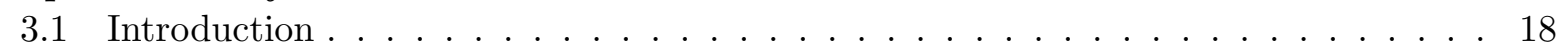

3.2 Global and Piecewise Interpolation . . . . . . . . . . . . . . . . . . . . . 19

3.3 Knot Sequences Method . . . . . . . . . . . . . . . . . . . . . . . 22

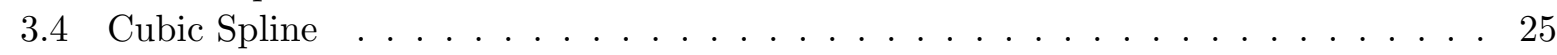

$\begin{array}{lll}4 & \text { Applications } & 28\end{array}$

4.1 Tracking Controller Design for Duffing Oscillator . . . . . . . . . . . . . . . . . . . 28

4.2 Excitation control with Direct Feedback Linearization Method . . . . . . . . . . . 38

4.2 .1 Control Design . . . . . . . . . . . . . . . . . . . . . . . 40

4.2 .2 Nonlinear Controller . . . . . . . . . . . . . . . . . . . . . . . . . . . . 41

4.3 Three Machine Nine Bus Test System . . . . . . . . . . . . . . . . . . . . . . 42

4.3 .1 Case 1 - Loss of Line . . . . . . . . . . . . . . . . . . . . 45 
4.3 .2 Case 2 - Loss of Load . . . . . . . . . . . . . . . . . . . 45

4.3 .3 Case 3 - Line to Ground Fault . . . . . . . . . . . . . . . . . . . 48

4.4 Spline Controller . . . . . . . . . . . . . . . . . . . . . . . . . . . . . . . . . 51

4.4 .1 Case 4 - Three Phase Fault . . . . . . . . . . . . . . . . . . . . . 53

4.5 Internal Variable Identifier . . . . . . . . . . . . . . . . . . . . . . . . 54

4.6 Controller Tuning - Genetic Algorithm . . . . . . . . . . . . . . . . . . . . . 58

4.6 .1 Case Study . . . . . . . . . . . . . . . . . . . . . . 59

4.7 Comparison between DFL Controller for One-Axis and Two-Axis Model . . . . . . 64

4.7 .1 Case 1 - Line to Ground Fault . . . . . . . . . . . . . . . . 65

4.7 .2 Case 2 - Three Phase Fault . . . . . . . . . . . . . . . . . . 65

4.7 .3 Comments . . . . . . . . . . . . . . . . . . . 66

$\begin{array}{lll}5 & \text { Summary and Conclusion } & \mathbf{7 0}\end{array}$

5.1 Conclusion and future work $\ldots \ldots \ldots \ldots \ldots \ldots$. . . . . . . . . . . . . . . . .

\begin{tabular}{ll}
\hline APPENDIX & 71
\end{tabular}

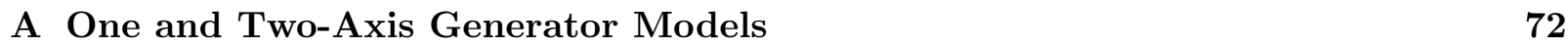

\begin{tabular}{ll}
\hline References & 75
\end{tabular} 


\section{List of Figures}

2.1 Periodic response of the Duffing Oscillation $\ldots \ldots \ldots \ldots \ldots$

2.2 Chaotic response of the Duffing Oscillation $\ldots \ldots \ldots \ldots \ldots$

2.3 Sensitivity to initial condition $\ldots \ldots \ldots \ldots \ldots \ldots \ldots$

$2.4 \quad$ Three Machine Nine Bus Test System with Spline Controller . . . . . . . . . . . . 17

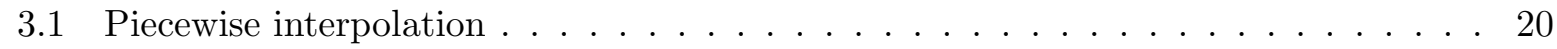

3.2 Global and piecewise interpolation . . . . . . . . . . . . . . . . . . 22

3.3 Global and piecewise interpolation $\ldots \ldots \ldots \ldots \ldots \ldots$

4.1 Uncontrolled response $\ldots \ldots \ldots \ldots$

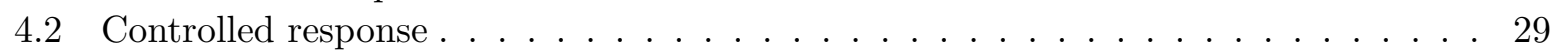

4.3 Controller signal $\ldots \ldots \ldots \ldots \ldots \ldots$

4.4 (a)-EFL controller (b)-spline controller $\ldots \ldots \ldots \ldots \ldots \ldots \ldots$

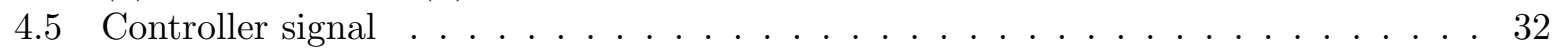

4.6 State trajectories for different polynomial degree $\ldots \ldots \ldots \ldots$. . . . . . . . . 33

4.7 Periodogram between DFL and cubic spline . . . . . . . . . . . . . . . . . . . . 37

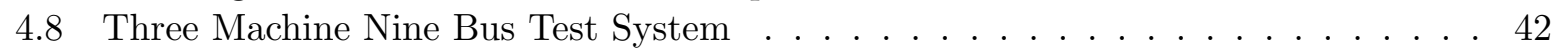

4.9 Schematic block diagram for spline-based controller. . . . . . . . . . . . . . . . . 44

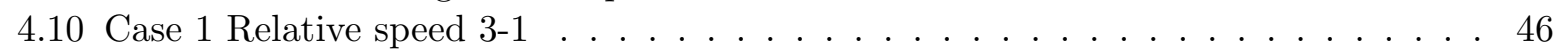

4.11 Case 1 Relative angle $3-1 \ldots \ldots \ldots$. . . . . . . . . . . . . . . . . 46

4.12 Case 2 Relative speed $2-1 \ldots \ldots \ldots$. . . . . . . . . . . . . . . . 47

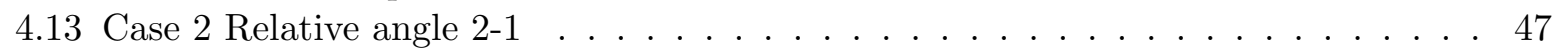

4.14 Case 3 Relative angle $2-1 \ldots \ldots \ldots$. . . . . . . . . . . . . . . . . 48

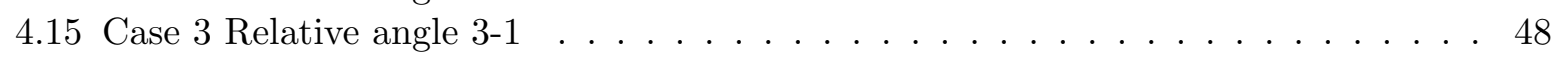

4.16 Case 3 Controller signals for generator $1 \ldots \ldots \ldots$. . . . . . . . . . . 49

4.17 Case 3 Controller signals for generator 1 (detail) $\ldots \ldots \ldots$. . . . . . . . . . 49

4.18 Case 3 Controller signals for generator 1 (detail) $\ldots \ldots \ldots$. . . . . . . . 50

4.19 Case 3 Controller signals for generator $2 \ldots \ldots \ldots \ldots$. . . . . . . . . 50

4.20 Case 3 Controller signals for generator $3 \ldots \ldots \ldots \ldots$. . . . . . . . . 51

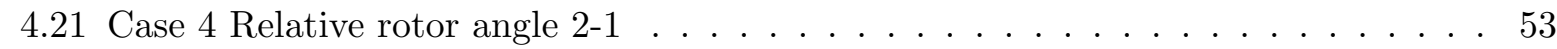

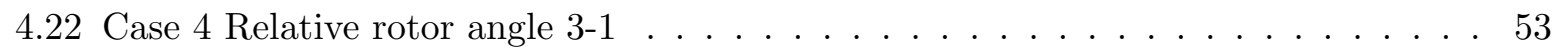

4.23 Internal variable identifier block diagram . . . . . . . . . . . . . . . . . . . 54

4.24 Direct and quadrature current for machine $1 \ldots \ldots$. . . . . . . . . . . 56 
4.25 Direct and quadrature current for machine $3 \ldots \ldots \ldots \ldots$

4.26 Speed of the machines $\ldots \ldots \ldots \ldots \ldots \ldots \ldots$

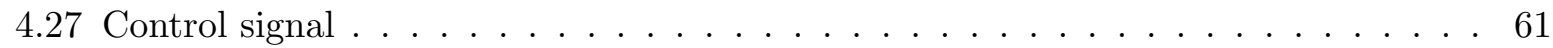

4.28 Control signal . . . . . . . . . . . . . . . . . . . . . . . . 61

4.29 Control signal . . . . . . . . . . . . . . . . . . . . . . . . . . 62

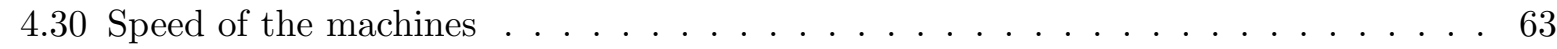

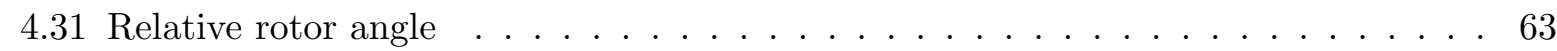

4.32 Comparison between relative rotor angle $\ldots \ldots \ldots \ldots$. . . . . . . . . 66

4.33 Comparison between control signal . . . . . . . . . . . . . . . . . . 66

4.34 Comparison between relative rotor angle $\ldots \ldots \ldots \ldots$. . . . . . . . . . 67

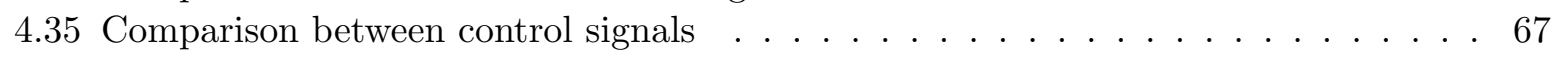

4.36 Effect of saturation block on first controller signal $\ldots \ldots \ldots$. . . . . . . . . 68

4.37 Effect of saturation block on second controller signal $\ldots \ldots \ldots$. . . . . . . . . . 69

4.38 Effect of saturation block on third controller signal $\ldots \ldots \ldots \ldots$ 


\section{List of Tables}

4.1 Controller effort with spline-based controller . . . . . . . . . . . . . . . . . . . . 35

4.2 Power spectral density with spline-based controller . . . . . . . . . . . . . . . . 36

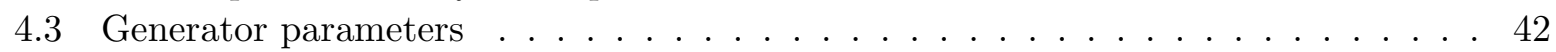

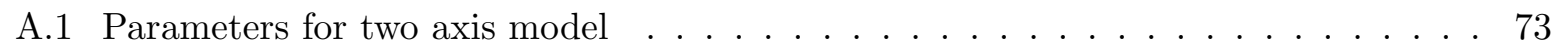

A.2 Parameters for one axis model $\ldots \ldots \ldots \ldots \ldots$. . . . . . . . . . . . 74 


\section{Chapter 1}

\section{Introduction}

\subsection{Motivation}

Significant progress has been made during the past few years on modelling and control design for systems. Generally, systems can be classified in several ways such as linear-nonlinear, time variant-time invariant, single input-single output, multiple input-multiple output, deterministicprobabilistic. It is usually tedious to design a controller for a nonlinear system. One way is to obtain the linearized model and based on the linearized model, the controller is designed. Linearization can be done around one operating point or several operating points.

A new nonlinear controller design is presented based on approximation techniques with splines. The controller is tested for different nonlinear systems. Here, the main objective is to see the effectiveness of the controller and compare it to conventional design methods.

In this thesis, the term Nonlinear Systems refers to either the Duffing Oscillator or power systems. The nonlinear oscillator itself is simple but it has all the characteristics of a complex nonlinear system such as chaotic oscillation. Nonlinear mathematical model for this system is called Duffing Oscillator.

Based on the model, nonlinear controllers are obtained and implemented. The objective of controller design is to drive the states of the system into a specific trajectory path. The problem is defined as tracking problem. Basic objective is to study the effectiveness of controller in comparison with conventional methods. 
The second system investigated is a power system. In the last decades power systems have grown largely in size and complexity. The complexity is not only in the system itself, but also in the amount of information that is needed to be monitored and processed in order to have the desired performance. Thousands of generators are connected together through the grid which is mainly high voltage AC transmission lines. These generators are nonlinear electromechanical devices that need to run synchronously and remain stable.

Transients in the power system are analyzed using many levels of modelling details. The model needs to be accurate enough to grasp the characteristics of transients. Mathematical formulation is governed by differential-algebraic equations that describe the dynamics of the generators in connection with the grid. The dynamic performance is influenced by machines and the controllers. Following large system disturbances, some synchronous generators may swing enough to lose synchronism with the system. Controllers are preferably installed locally at the generators and directly affect the stability of the overall grid.

From this point of view, control is vital to maintain the stability of the interconnected power system. Because of this need, control structures are becoming more pervasive and numerous, guaranteeing the stability of system over a wide range of operating points. Information about the system is also distributed over a wide geographic area, and hence the only feasible control structure is a decentralized one. Moreover the controllers need to be nonlinear in order to act properly for larger operating regions. Spline approximation techniques are applied in controller design for generator side. Controller performance is compared with conventional control method by Direct Feedback Linearization (DFL) method.

DFL has been applied to power systems previously but only for simplified generator models such as the "one-axis" model. Here the "two-axis" generator model is used. The designed DFL is a decentralized nonlinear controller with internal state identifier. The identifier is used to construct the internal variables of the machine which cannot be measured directly. 


\subsection{Approach}

In this thesis, MATrix LABoratory (MATLAB) is used for simulation, symbolic computation and coding. In brief, MATLAB is a programming environment that is supported by a variety of toolboxes comprising source code. Sophisticated algorithms in high level languages are provided by these toolboxes. Several toolboxes such as control toolbox, signal processing toolbox, genetic algorithm toolbox, spline toolbox, and power analysis toolbox are used. These toolboxes are a collection of algorithms for control implementation, signal and system processing, data fitting, interpolation, spline functions, and power system analysis.

\subsection{Overview}

The organization of this thesis is as follows. Literature survey is given in Chapter 2. Two independent classes of nonlinear systems are covered separately, namely oscillator and power systems. Controller design is considered for both cases. In Chapter 3 basic definitions for approximation technique with spline method, mainly cubic splines, are given. Chapter 4 discusses the application of spline methods in controller design. First, duffing oscillator is covered. Tracking controller design is tested with spline-based controller and compared to conventional control method. Second the electric power system is analyzed. Application of the spline approximation is tested in the excitation control loop of the synchronous generator. Classical three-machine nine-bus test system with two-axis generator model is considered.

Internal Variable Identifier is also presented in Chapter 4. The identifier is used to obtain the internal variables of the machine which cannot be measured directly. At the end comparison between excitation controller with DFL controller for two-axis and one-axis model is covered. Chapter 5 includes a summary of studies done and discussion of possible future work. 


\section{Chapter 2}

\section{Literature Survey}

\subsection{Nonlinear Oscillators}

\subsubsection{Definitions}

Linear systems are ideal models and mainly have simple mathematical equations with explicit solutions. Some characteristics of these systems are invariance under scaling, additivity, and frequency fidelity which can be defined as follows [15].

Invariance under scaling - If the input $u$ gives the output $y$, then $\alpha u$ gives $\alpha y$.

Additivity - If the input $u_{1}$ and $u_{2}$ give outputs $y_{1}$ and $y_{2}$ respectively, then $u_{1}+u_{2}$ gives the output $y_{1}+y_{2}$.

Frequency Fidelity - A component in a reference or disturbance signal with frequency $f$ affects the control error only at the frequency $f$.

In physical systems these characteristics are not valid due to nonlinearity which bring a significant difference between linear and nonlinear systems. Practical engineering systems are inherently nonlinear. From this point of view, accurate modelling of system has essential role for controller design techniques. Theoretical study of this field is very subtle and often need to be supplemented by numerical techniques. 
The torsion pendulum is a physical example of nonlinear oscillators and shows all the basic features of a dynamical system. The behavior of nonlinear pendulum has been explored by different researchers. Though it is an example of a simple system it can demonstrate different behaviors. The mathematical model of the nonlinear oscillator is known as Duffing Oscillator. Chaotic oscillations are of interest and it has been used as a basis for mathematical models for many systems. But this dynamics, even for simple systems, are not always predictable. This motion is called chaotic and has been investigated [32, 6]. Note that chaotic behavior of the system is not due to random external influence. Examples of such systems can be found in some chemical reactions, mechanical and electrical oscillator. One characteristic of a chaotic system is that although the motion is deterministic (given an exact initial condition, its future motion can be predicted mathematically) it is non-periodic, which means that the motion of pendulum is not repeated in time. The other characteristic is the extreme sensitivity to initial states which leads into unpredictable response of the system. It is essential to know when the system shifts toward a chaotic mode and how to prevent that with help of controllers. Therefore, the main objective is to train the controller to drive a chaotic system to follow a specific trajectory and maintain the system at that specific target. Before getting into more details in system description and control methods, some classical definitions for different types of oscillations are explained in the following 6 .

Periodic one motion - If the motion of pendulum (output response of the system) repeats after one driving cycle the motion is called period one motion. This will happen at relatively high frequency.

Periodic two motion - If the motion of pendulum (output response of the system) repeats after two driving cycles the motion is called period two motion.

Chaotic motion - If the motion of pendulum does not repeat its path the response is called chaotic. Note that chaos and random behavior are different. For random processes some statistical measures of the parameters are known but for chaotic behavior there are no random or unpredictable inputs. So all the parameters and initial conditions are considered to be known for chaotic behavior of the system [6]. 
Here, the main objective is to design a controller which can bring a chaotic oscillation into a periodic orbit and capable to order the chaos. The controller design objective can be expressed as a tracking problem. The design of nonlinear and linear controllers for driving chaotic trajectories of chaotic motion to its periodic orbits are covered. Both linear and nonlinear control methods have been used in this research area. A literature survey mainly related to the Duffing oscillator is presented next

Lefeber et al. [23] designed a state feedback controllers for a chaotic dynamical system and presented the bounded feedback controller for trajectory tracking in forced Duffing equation. The controller was defined globally with help of Lyapunov function. The trajectory tracking was established for any initial state condition and any desired trajectory. It is clear that selecting different Lyapunov functions may lead into different controllers with different convergence rate. So it is up to designer to select an appropriate Lyapunov function.

Conventional nonlinear feedback controller by applying Lyapunov direct method has also been applied by Dong et al. 9] for continuous chaotic system where the target position is an equilibrium point of chaotic system. They also showed that conventional canonical feedback control technique can control the chaotic trajectory of a continuous time nonlinear system, specifically Duffing oscillator, to converge into its equilibrium and also multi-periodic orbits.

From a continuation of Dong et al. [10] work the adaptive feedback controller was developed based on Lyapunov stability theory for an uncertain chaotic Duffing oscillator.

Linear feedback controllers have also been applied to forced Duffing oscillators. Linear feedback control laws are simpler to implement in practice than nonlinear controllers. Referring to Jiang et al. [18, linear controllers are often less sensitive to sensor errors and inherently robust against measurement errors. State feedback and nonlinear observer based output feedback control laws were also obtained and showed that tracking error converges to zero.

Chen et al. [7] investigated this problem by establishing a linear feedback control law for nonlinear oscillation. They presented a methodology based on input-output linearization method. The controller were modified by locally linearizing the nonlinear control law in small neighbor- 
hood of the control goal. They also showed through simulation that the controller law is robust to model errors.

In this thesis, EFL controller is obtained for forced Duffing oscillator based on Exact Feedback Linearization method. Then spline technique is used to approximate the nonlinear controller by piecewise polynomials. Order of polynomials can be changed easily. It will show that the spline based controller can be trained to drive a chaotic system to follow a certain trajectory [19].

\subsubsection{Mathematical Model}

Forced Duffing oscillator is a well-known model for a mass-spring mechanical system which contains hardening spring, viscous friction, and a periodic external force [21]. It was first introduced by Duffing in 1918 and formulated as a second-order non-autonomous ordinary differential equation with nonlinear term in form of cubic stiffness.

$$
\ddot{x}+p_{1} \dot{x}+p_{2} x+p x^{3}=q \cos (\omega t)
$$

Parameters are defined as follow

$$
\begin{array}{rll}
p_{1} & \ldots & \text { Damping coefficient } \\
p & \ldots & \text { Constant coefficient for nonlinear part } \\
p_{2} & \ldots & \text { Constant coefficient for linear part } \\
t & \ldots & \text { Time } \\
q & \ldots & \text { Amplitude of external driving force } \\
\omega & \ldots & \text { Frequency of external driving force }
\end{array}
$$

In physical system the parameters $p_{2}, p$ are determined by pendulum dimensions. Changes in damping coefficient, forcing amplitude and forcing frequency yield different responses of the system in terms of period 1, period 2, and chaotic behavior. Output responses of the system show different dynamic behaviors of the system. The equation can be written in form of two first-order ordinary differential equations or in affine form

$$
\begin{aligned}
& \dot{x_{1}}=x_{2} \\
& \dot{x_{2}}=-p_{1} x_{2}-p_{2} x_{1}-p x_{1}^{3}+q \cos (\omega t)
\end{aligned}
$$


where

$$
\begin{aligned}
& x_{1}=x \\
& x_{2}=\dot{x}
\end{aligned}
$$

and the output is taken as $x_{1}$. The main interest is to train the controller in a way that removes the chaotic system behavior.

For dynamic behavior of the Duffing oscillation, consider a numerical example as follows. Introducing $y=\dot{x}$, Duffing equation is given as two first order differential equations in affine form.

$$
\left\{\begin{array}{c}
\dot{x}=y \\
\dot{y}=-p_{1} x-x^{3}-p y+q \cos (\omega t)
\end{array}\right.
$$

Periodic and chaotic behavior can be obtained by choosing parameters of Duffing equation as $p=0.4, p_{1}=-1.1, \omega=1.8$. Changing external driving force or $q$ can yield into periodic and chaotic system behavior. Figure 2.1 shows period- 1 motion after 25 seconds with $q=0.620$, and Figure 2.2 shows chaotic motion with $q=1.8$. As it can be seen from Figure 2.1, the response

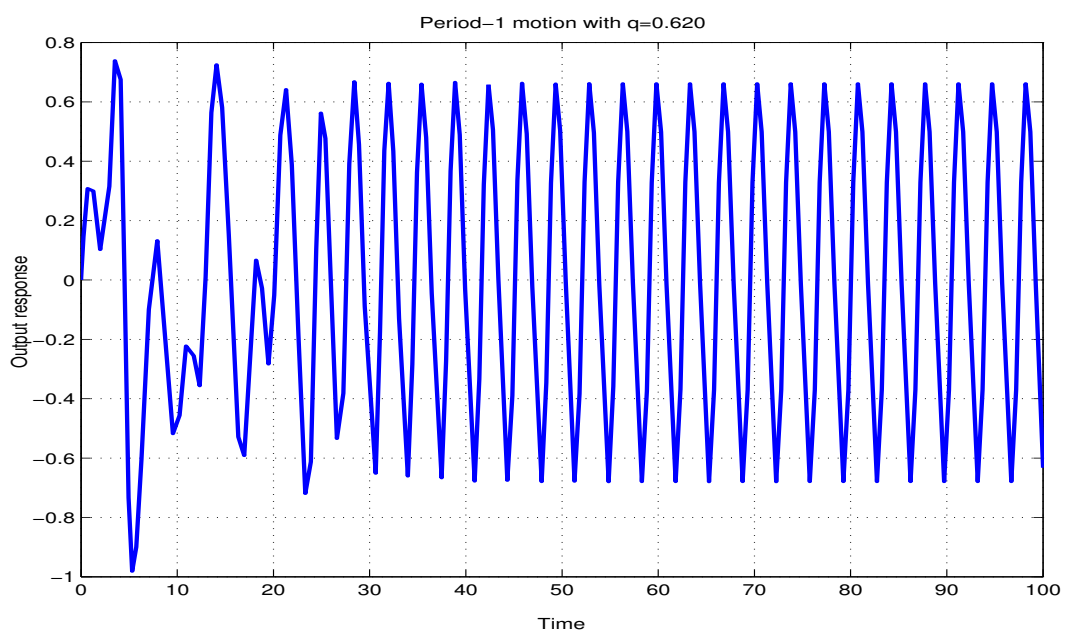

Figure 2.1: Periodic response of the Duffing Oscillation

repeats after one driving cycle that express period-1 cycle.

Another important characteristic of Duffing oscillator is high sensitivity of output response to its initial conditions. A Duffing oscillation formulated by equations 2.2 and 2.3 where $x_{1}=x$ and $x_{2}=\dot{x}$. The system's output is taken as $x_{1}$. Consider the Duffing equation with specific values $p=0.4, p_{1}=-1.1, \omega=1.8, q=2.1$, and the initial conditions $x_{10}=x_{20}=1$. The state response 


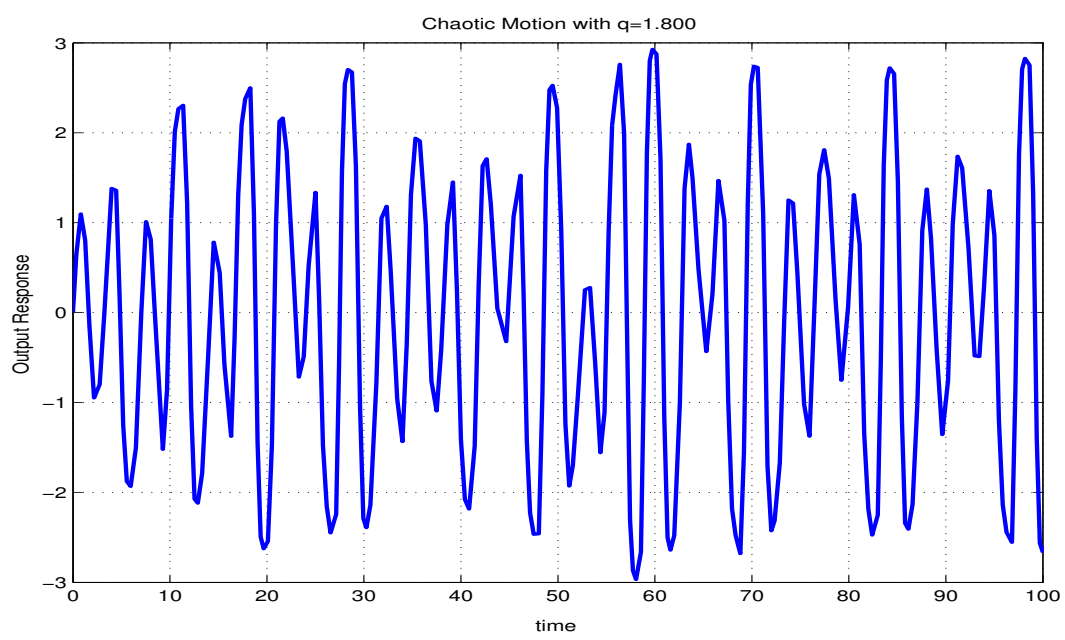

Figure 2.2: Chaotic response of the Duffing Oscillation

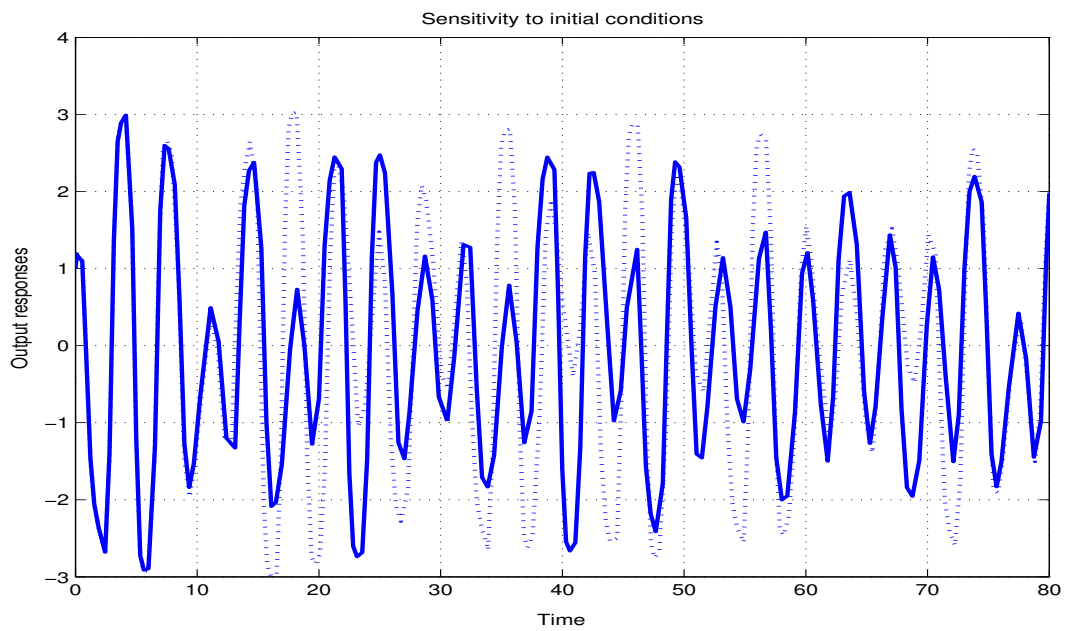

Figure 2.3: Sensitivity to initial condition

with these conditions is shown in Figure 2.3 (solid line). Additionally, the state response for the initial conditions of $x_{10}=1$ and $x_{20}=1.01$ is given (dotted line). The high sensitivity for very small variation in initial conditions can be seen clearly from the output response of the system.

\subsubsection{Problem Description}

From control point of view, Duffing oscillator as explained in 2.1) is considered as uncontrolled oscillator. Depending on parameters, the solution is a periodic or chaotic behavior. Controller signal can be understood as a force (torque) applied to the uncontrolled Duffing equation. 
So, the formulation can be modified as

$$
\ddot{x}+p_{1} \dot{x}+p_{2} x+p x^{3}=u+q \cos (\omega t)
$$

The proposed controller needs to satisfy the global tracking problem. This tracking trajectory is independent of starting point of original system and also chaotic behavior of the system. Mainly, tracking problem is achieved by means of feedback controllers that automatically act as error correction mechanisms. This feedback controller can be obtained by nonlinear control methods such as Lyapunov direct method. Another method is based on exact linearization. The method itself has great attraction in nonlinear control [17]. The main idea of this method is to construct a coordinate transformation and a feedback control law such that input-output or the input-state relationship of the close loop system, in the new coordinates, is linear. This technique has been applied to chaotic systems such as Duffing and Lorenze systems.

The approach considered here is different in the point that spline theory is applied to approximate the original nonlinear controller. Nonlinear controller is approximated by piecewise polynomial with specific order of polynomial. There are several parameters involved in this approximation methods such as the number of sampled data, knots position, different types of spline and the order of polynomial for interpolation method. This study is mainly focused on numerical experiments based on nonlinear Duffing oscillator. For the proposed controller, it is important to see whether the controller can bring the state of the system to a desired path and how much effort and energy it takes to fulfill this task.

\subsubsection{Controller Design}

The following gives a brief numerical example related to EFL method. Consider a nonautonomous system with a control law $u$, given by

$$
\dot{x}=f(x, t)+u
$$

Without control law $u$, the system has chaotic behavior for a given set of system parameters. The objective is to design a control law $u$ to order the chaotic behavior. Assume that $x_{d}(t)$ is a desirable path that can be a fixed point or periodic orbit. Defining the error signal as $e(t)=x(t)-x_{d}(t)$, the control design should result in a control law $u$ such that $e \rightarrow 0$ as $t \rightarrow \infty$. 
For a single input single output system given as

$$
\begin{aligned}
\dot{x} & =f(x)+g(x) u \\
y & =h(x)
\end{aligned}
$$

where

$$
\begin{gathered}
f(x)=\left(\begin{array}{c}
x_{2} \\
-p_{1} x_{2}-p_{2} x_{1}-p x_{1}^{3}+q \cos (\omega t)
\end{array}\right) \\
g(x)=\left(\begin{array}{l}
0 \\
1
\end{array}\right) \\
h(x)=x_{1}
\end{gathered}
$$

The vectors $x, u$ and $y$ are the state, input and output vector, respectively. The vectors $f(x)$ and $g(x)$ are smooth and real functions. The system is defined to have relative degree $n$ at point $x$ if it satisfies the following conditions

$$
\begin{aligned}
L_{g} L_{f}^{k} h(x) & =0 \quad \forall \quad k<n-1 \\
L_{g} L_{f}^{n-1} h(x) & \neq 0
\end{aligned}
$$

The notation $L_{f} \lambda(x)$ is called Lie derivative or derivative of $\lambda$ along the state trajectory and it is defined as $\frac{\partial \lambda}{\partial x} f(x)$ [17. Repeated Lie derivatives are defined by 2.15, 2.16, and 2.17). Also if $g$ is another vector field then (2.18). There are some basic notation related to Lie derivative which are given as follow

$$
\begin{array}{r}
L_{f}^{0} h(x)=h(x) \\
L_{f}^{2} h(x)=L_{f} L_{f} h(x)=\frac{\partial L_{f} h}{\partial x} f(x) \\
L_{f}^{k} h(x)=L_{f} L_{f}^{k-1} h(x)=\frac{\partial\left(L_{f}^{k-1} h\right)}{\partial x} f(x) \\
L_{g} L_{f} h(x)=\frac{\partial L_{f} h}{\partial x} g(x)
\end{array}
$$


The set of equations for single input and single output system is input-output linearizable if it has finite relative degree $n$ with respect to the output. The relative degree is the number of times the output function needs to be differentiated before the input explicitly appeares. The control law in general form is given by [17, 21]

$$
u=\frac{-L_{f}^{n} h(x)+x_{d}^{(n)}}{L_{g} L_{f}^{n-1} h(x)}-\frac{\sum_{i=1}^{n} C_{i-1}\left(L_{f}^{i-1} h(x)-x_{d}^{i-1}\right)}{L_{g} L_{f}^{n-1} h(x)}
$$

The pole placement method or linear quadratic regulator (LQR) can be used in order to specify the coefficients $C_{i-1}$ for $i=1 . . n$. Relative degree for the Duffing oscillator system is obtained as

$$
L_{g} L_{f} h(x)=\frac{\partial L_{f} h(x)}{\partial x} g(x)
$$

Where

$$
L_{f} h(x)=\frac{\partial h(x)}{\partial x} f(x)=x_{2}
$$

The Lie derivative will become $L_{g} L_{f} h(x)=1$ and shows that system has a relative degree of second. The control law is given by

$$
\begin{gathered}
u=\frac{-L_{f}^{2} h(x)+x_{d}^{(2)}-\sum_{i=1}^{2} C_{i-1}\left(L_{f}^{i-1} h(x)-x_{d}^{(i-1)}\right)}{L_{g} L_{f}^{2-1} h(x)} \\
u=\frac{-L_{f}^{2} h(x)+x_{d}^{2}-C_{0}\left(L_{f}^{0} h(x)-x_{d}^{0}\right)-C_{1}\left(L_{f}^{1} h(x)-x_{d}^{1}\right)}{L_{g} L_{f} h(x)}
\end{gathered}
$$

Use following Lie derivatives in 2.23

$$
\begin{aligned}
L_{f}^{2} h(x) & =-p_{1} x_{2}-p_{2} x_{1}-p x_{1}^{3}+q \cos (\omega t) \\
L_{f} h(x) & =x_{2} \\
L_{f}^{0} h(x) & =x_{1} \\
L_{g} L_{f} h(x) & =1
\end{aligned}
$$

Finally, the controller law is obtained as

$$
u=p_{1} x_{2}+p_{2} x_{1}+p x_{1}^{3}-q \cos (\omega t)+x_{d}^{(2)}-C_{0}\left(x_{1}-x_{d}^{(0)}\right)-C_{1}\left(x_{2}-x_{d}^{(1)}\right)
$$

In Chapter 4 tracking problem for Duffing oscillator will be covered by using spline techniques. 


\subsection{Power Systems}

\subsubsection{Power System Dynamics}

The electric power system is a large interconnected system whose structure changes constantly due to changes in loading conditions, planned or unplanned line-outages, disturbances, and installation of new devices and apparatus to maintain a minimum level of reliability. Information about the system is distributed over a wide geographic area, and hence the only feasible control structure is a decentralized one. Electric machines are part of this nonlinear system and therefore a challenging area for implementation of nonlinear control techniques to enhance the stability of the system.

Dynamics of electric machines exhibit significant non-linearities. Besides, it is not always the case that access to all state variables of system is given. The parameters of the system may also vary significantly from their nominal values. So it is necessary for nonlinear controllers to include an algorithm to provide state estimation and parameter identification in order to overcome this problem [22]. Recently, several methods have been used for enhancing power system stability. Literature survey based on power system stability enhancement is given in the following.

An overview related to nonlinear control design for generators is covered by Taylor [28]. The most common control designs for electric machines, for application requiring high dynamic performance, are based on forms of exact linearization. As mentioned by Taylor "Not all nonlinear systems can be controlled in this fashion; the applicability of exact linearization is determined by the type and location of the model nonlinearities."

Among nonlinear controller methods direct feedback linearization is an attractive methodology that can be applied to the excitation system of synchronous generators. Excitation control systems have been improved since the 60's [28]. The control is based on mathematical modelling of the synchronous machine. So, the modelling for electric machines needs to be accurate enough to grasp the essential characteristics relevant to control design, especially with nonlinear methods as direct feedback linearization method.

Direct feedback linearization has been investigated by Wang et al. [30, 31]. They applied 
DFL controller method to one-axis model generator. The main concern is to prevent an electric power system losing synchronism after a large sudden fault on the system. Also, achieving the good post fault regulation of generator terminal voltage is their concern.

Application of nonlinear decentralized control to large scale power systems has been presented by Hill et al. [16]. They proposed nonlinear bounds of generator interconnections in order to achieve less-conservative control gains while maintaining the stability of the system.

Loss of synchronism can be detected by distance or gap between rotor angle oscillation with respect to time. If the rotor angle is increasing in comparison to the rest of the machines, the system is considered unstable. For stability problem, usually it is more convenient to work with relative rotor angle rather than absolute angle. More description related to synchronism and first swing problem can be found in reference [24].

In this thesis, first DFL controller is obtained for two-axis generator model. Based on nonlinear controller, spline techniques are used to approximate the controller by polynomials of different degrees. It will show that the spline-base controller can enhance the stability of the system [20].

Generator models can be classified as classical, transient and subtransient. For transient classification there are two models with one-axis or two-axis. A complete description for machine modelling is given by Sauer et al. [26]. Mathematical modelling for one-axis and two-axis generators are covered in the Appendix.

\subsubsection{Stability Enhancement}

Power systems are large-scale nonlinear systems prone to faults and disturbances. The major concern for the system during fault periods is preserving the synchronism of the generators and stability of the system. One controller device, known as Power System Stabilizer (PSS), is designed mainly by using linear control theory. The plant is linearized of a few operating points and the controller is designed based on the linearized models. The drawback of this design is that by changing the operating point of the system, the controller may not work effectively. 
The excitation control plays an important role in preserving the stability of the system. Here, nonlinear controllers with Direct Feedback Linearization is applied. Linear control methods have also been applied previously. The major problem with linear controller is the limitation of operating region. Due to a large disturbance in the system, the operating condition may change and controllers may not act properly with new operating conditions.

From two-axis model dynamics it is obvious that the synchronous generator is nonlinear through the excitation loop. Some of the control design requirements are high dynamic performance.

It is assumed that the controller is designed as a part of generator excitation system design. Because of the physical limitations in the system, the output of controller is limited to a certain level and this fact has influence on the overall system performance.

Similar methodology based on feedback lineaization has been applied to single machine infinite bus with one-axis generator [30, 31]. Simulation results show that controller is capable of stabilizing the system even when subjected to large disturbances.

\subsubsection{Problem Description}

As mentioned before, transient stability is the major concern in operating large-scale power systems. Stability enhancement is always sought since the power system is constantly undergoing structural and loading condition changes.

One way of enhancing stability is through the DFL excitation system. The spline techniques are used to approximate the excitation controller. The fundamental idea behind approximation theory is to resolve a complex nonlinear function, which is called target function, with a simpler function called approximants in the form of piecewise polynomials. The number of parameters that need to be specified for this polynomial are determined by the complexity of the approximation process. However, it does not necessarily agree with computational complexity. The objective is to study the effect of control by spline approximation and to investigate the behavior of the approximated controller while the system is subjected to disturbances. 
The spline approximations are in different forms and bases. Two commonly used spline functions are cubic spline and B-spline. Both types of spline approximation are used in excitation controllers. By applying faults, the performance of two approximant controllers are compared. The comparison is done in order to test how well the controllers are able to damp generator rotor oscillations and how fast stability of the system for post fault condition can be restored.

In constructing the approximants, the other issue is breaking points or free knots which need to be designed. There are several methods mentioned by de Boor that can lead into break points from free sequences of knots [3]. But it is not always easy to find partitions that give a good approximation from free knots. Once new break points are computed, approximated functions are constructed with spline functions based on these breaking points. As mentioned in [3] "when interpolating with spline of order $K$, one good method is to knot averages from the augmented knot sequence."

Generally, controller design for the excitation loop with DFL method concept has two stages. In the first part, nonlinear compensation is derived which explicitly cancels the non-linearities present in synchronous generator without any specific control objective. In the second part, linear compensation is derived on the basis of the resulting linear dynamics of the pre-compensated machine to achieve some particular control objective.

Nonlinear approximation theory is applied in DFL Controller with spline techniques. The controllers are approximated in different forms as cubic spline and B-spline. The approximated controller is tested for the three machine nine bus system [2]. Schematic view of the system is shown in Figure 2.4. Spline controllers are specified for each area by a controller block locally at the generator's side. Each area is specified by a circle which encloses generator and controller for excitation, measurable outputs are given as inputs to the controller and control signal is fed back to the excitation system of the generator. 


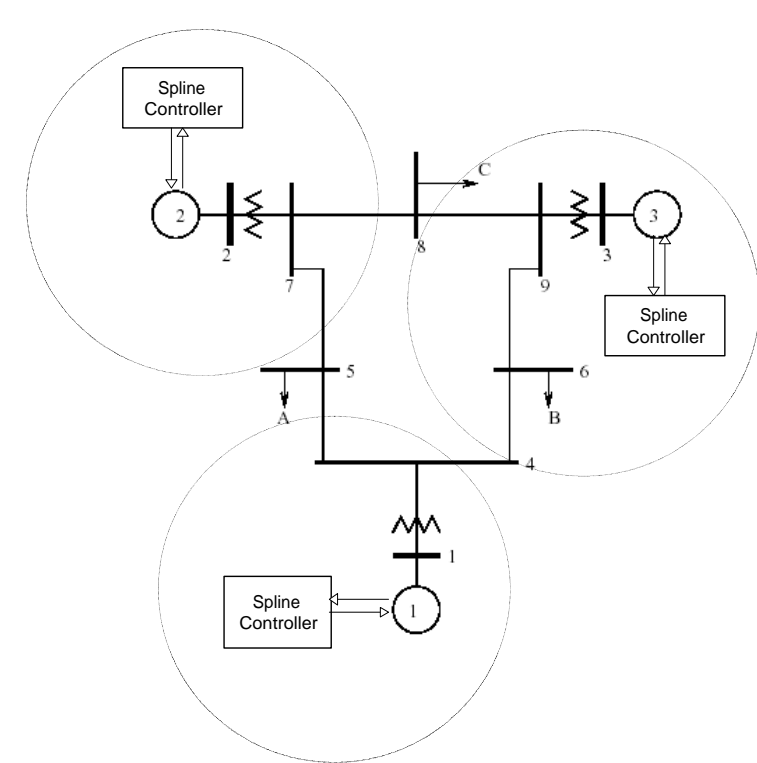

Figure 2.4: Three Machine Nine Bus Test System with Spline Controller 


\section{Chapter 3}

\section{Spline Theory}

\subsection{Introduction}

Interpolation and the cubic spline technique are covered in this section. These methods have numerous applications in engineering problems, especially in analysis of sampled data points. Generally, interpolation is used to estimate the value of a function between known data points. The main objective is to fit a curve or set of curves to sampled data. Interpolation can be divided into two classes global and piecewise interpolation. The task consists of finding an approximate function instead of the original complicated function. A typical curve fit or global interpolation involves forming one polynomial through all set of data points. By using only one polynomial for all set of points the order of the polynomial will become too high. Although the result is a smooth curve, it is not well suited for engineering applications because it is prone to high oscillation and overshoot at adjoining points. The order of polynomial is defined as the number of points minus one. Increasing the order does not necessarily increase the accuracy of interpolation, especially in polynomial interpolation.

The other method is piecewise interpolation. The method is to match the data points using a lower order polynomial between each pair of adjacent data points. If a first-degree polynomial is used, it is called linear interpolation. For second and third order polynomials it is called quadratic and cubic polynomial, respectively. Piecewise interpolation allows each segment to have a unique polynomial while guaranteeing global smoothness in the interpolated function up to a certain order of its derivative. As an example cubic spline interpolation has a polynomial of third degree for each segment and is continues up to the second order derivative. Mathematical formulation 
for cubic spline and the procedure to derive the polynomial coefficients are given in detail.

\subsection{Global and Piecewise Interpolation}

Consider a smooth nonlinear function $f(x)$ where $x$ takes values in the interval $[a, b]$. Let $x_{0}$ be a point in this interval. Local approximation of smooth function is usually done with polynomials. One way to approximate the function $f\left(x_{0}\right)$ is to use a truncated Taylor series expansion $\sum_{j=0}^{n} \frac{(x-a)^{j}}{k !} D^{j} f(a)$ where $D^{j} f(a)=\left.\frac{\partial^{j} f(x)}{\partial x^{j}}\right|_{x=a}$. For larger number of points, passing a single polynomial through all of them, yields a high order interpolant which is expensive to determine and evaluate. Note that in this case, polynomial goes through the data points but it may oscillate between data points. Better method is to subdivide the whole range $[a \ldots b]$ of approximation into small subintervals as $a=\tau_{1}<\tau_{2}<\ldots \tau_{n-1}<\tau_{n}=b$.

The objective is to fit a polynomial to each subinterval. Splines are a special set of piecewise polynomials and it provides a technique for obtaining a smoother interpolation formula [12, 13. A commonly used spline technique is the cubic spline. Cubic spline provides an interpolant, that is twice continuously differentiable. In addition spline method can be extended to two or three dimensions by sequencing one-dimensional interpolation. It also allows curvature between points and has continuous first and second derivatives on the interval $[a, b]$. The main advantage of piecewise polynomial interpolation, in comparisom to global interpolation, is that a large number of data points can be fit with low order polynomials which are aligned between each pair of knots controlling points. Cubic spline can be interpreted as the result of bending a flexible rod between a series of control points or knots. Figure. 3.1 shows a flexible beam using cubic spline curves which passes through a limited number of control points. Consider the cubic polynomial for one segment as

$$
z=a_{i}\left(\tau-\tau_{i}\right)^{3}+b_{i}\left(\tau-\tau_{i}\right)^{2}+c_{i}\left(\tau-\tau_{i}\right)+d_{i}
$$

The parameters are 


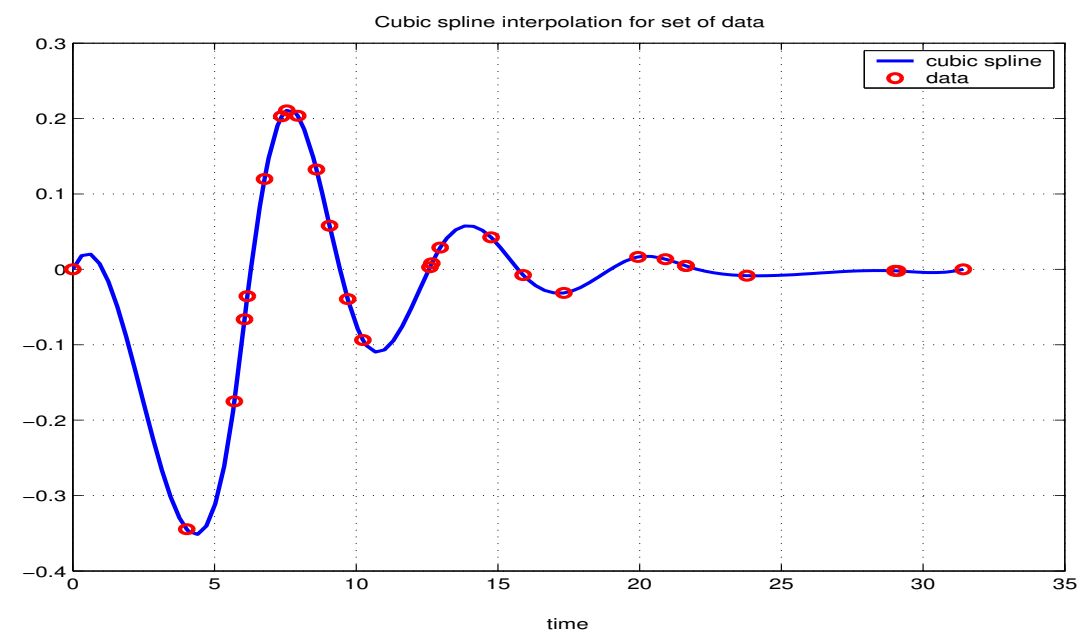

Figure 3.1: Piecewise interpolation

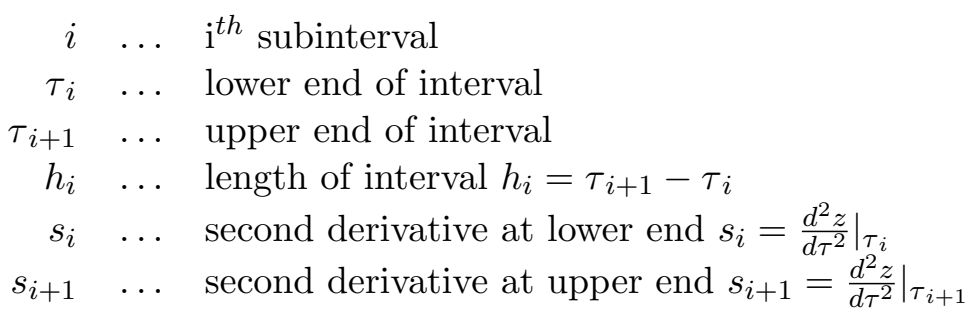

Abscissas $\tau_{i}$ are called knots or breakpoints at which interpolants change from one cubic polynomial to another. Polynomial coefficients $a_{i}, b_{i}, c_{i}$, and $d_{i}$ are specified with respect to $s_{i}$ and $s_{i+1}$ for each segment [14].

$$
\begin{aligned}
a_{i} & =\frac{s_{i+1}-s_{i}}{6 h_{i}} \\
b_{i} & =\frac{s_{i}}{2} \\
c_{i} & =\frac{z_{i+1}-z_{i}}{h_{i}}-\frac{2 h_{i} s_{i}+h_{i} s_{i+1}}{6} \\
d_{i} & =z_{i}
\end{aligned}
$$

The $s$ terms which contain $s_{1}, s_{2}, \ldots, s_{n-1}, s_{n}$ are obtained from solving the following set of equations. These recursive equations specify the slope of adjacent polynomials at each segment.

$$
h_{i-1} s_{i-1}+\left(2 h_{i-1}+2 h_{i}\right) s_{i}+h_{i} s_{i+1}=6\left(\frac{z_{i+1}-z_{i}}{h_{i}}-\frac{z_{i}-z_{i-1}}{h_{i-1}}\right)
$$

The right side of the above equation contains known values, which includes $z$ and $h$. From the left side, $h$ is known, so the equation can be solved for $s$ on each segment and coefficients for 
piecewise cubic polynomials can be computed for $i=2,3, \ldots, n-1$. To have a unique solution, number of equations must equal number of parameters to be determined. Note that (3.6) has $n-2$ equations and $n$ unknowns. Thus it needs to eliminate two unknowns $\left(s_{1}, s_{n}\right)$ which define end conditions on the shape of the spline. In brief, there are different types of cubic splines with different end conditions such as complete cubic spline, natural cubic spline [3, 4].

Polynomial coefficients as given in $(3.2)-3.5)$ are derived as follows. Matrix formulation is given by Wheatly and Gerald [14].

$H=\left(\begin{array}{cccccccccc}h_{1} & 2\left(h_{1}+h_{2}\right) & h_{2} & 0 & 0 & 0 & \ldots & 0 & 0 & 0 \\ 0 & h_{2} & 2\left(h_{2}+h_{3}\right) & h_{3} & 0 & 0 & \ldots & 0 & 0 & 0 \\ 0 & 0 & h_{3} & 2\left(h_{3}+h_{4}\right) & h_{4} & 0 & \ldots & 0 & 0 & 0 \\ \vdots & \vdots & \vdots & \vdots & \vdots & \vdots & \vdots & \vdots & \vdots & \\ 0 & 0 & 0 & 0 & 0 & 0 & \ldots & h_{n-2} & 2\left(h_{n-2}+h_{n-1}\right) & h_{n-1}\end{array}\right)$

$$
S=\left(\begin{array}{c}
s_{1} \\
s_{2} \\
\vdots \\
s_{n}
\end{array}\right)
$$

$$
Z=\left(\begin{array}{c}
\frac{6 z_{3}-6 z_{2}}{h_{2}}-\frac{6 z_{2}-6 z_{1}}{h_{1}} \\
\frac{6 z_{4}-6 z_{3}}{h_{3}}-\frac{6 z_{3}-6 z_{2}}{h_{2}} \\
\vdots \\
\frac{6 z_{n}-6 z_{n-1}}{h_{n-1}}-\frac{6 z_{n-1}-6 z_{n-2}}{h_{n-2}}
\end{array}\right)
$$

Equation is given as $H S=Z$ and the objective is to find $S$. Thus one needs to eliminate two unknowns $\left(s_{1}, s_{n}\right)$ which define end conditions on the shape of spline. These two end points can specify different types of cubic splines, $S$ is also referred to mass matrix. As an example in natural spline the second order derivative of splines at the end points are zero. Another type is clamped spline with the first order derivatives of the spline at the end points are set to be known values [3, 4]. 


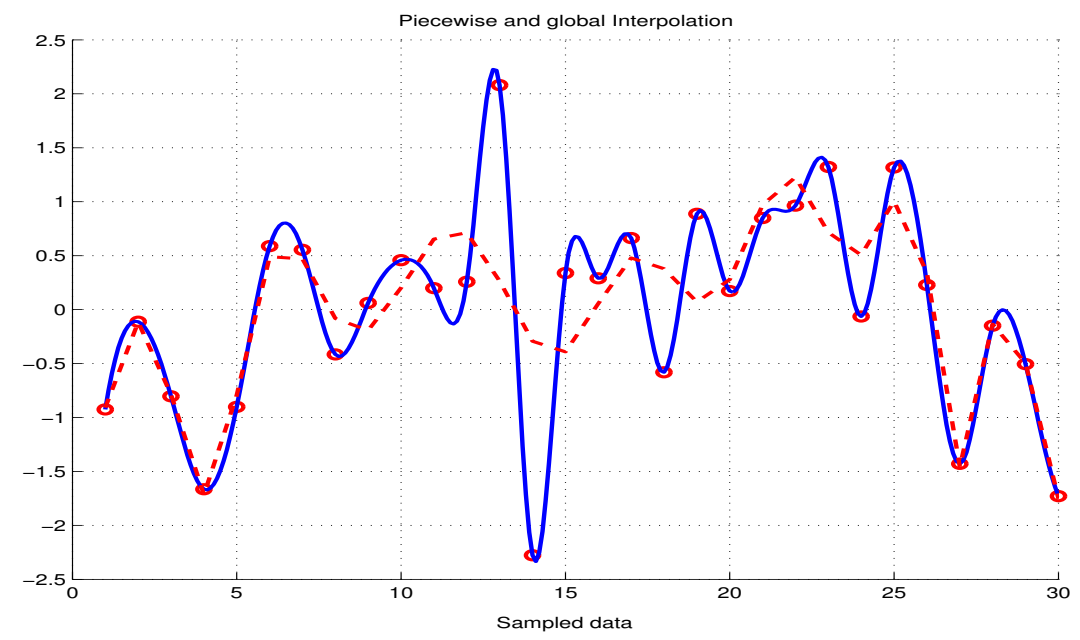

Figure 3.2: Global and piecewise interpolation

As a numerical example consider a random set of data, global and piecewise interpolation are applied to this random set. Figure 3.2 shows the comparison between global and piecewise interpolation. Global polynomial has relative high degree polynomial interpolant. Dashed line presents a global interpolation and solid line presents piecewise polynomial interpolation.

For low degree polynomial, global interpolation does not give exact match through break points as shown in Figure 3.3 and can not pass through all break points. Through a number of sampled data points which are taken arbitrarly, global polynomial interpolation is shown with dashed line and piecewise polynomial in shown with solid line. The global interpolation clearly shows that the approximant cannot pass through the control points and it gives a very different curve when compared to piecewise polynomial interpolation.

\subsection{Knot Sequences Method}

For cubic splines, the support basis function consists of four piecewise cubic sections. The end-points are aligning with the knots and sampled data are used for starting point for spline interpolation method. It is possible to specify where the spline interpolant should have its new break points and it is recommended as good interpolation method [3]. One good method is knot sequences method. The procedure is explained by numerical example.

Consider a nonlinear function $u=f\left(x_{i}\right), i=1,2, \ldots, n$ with bounded variation for each 


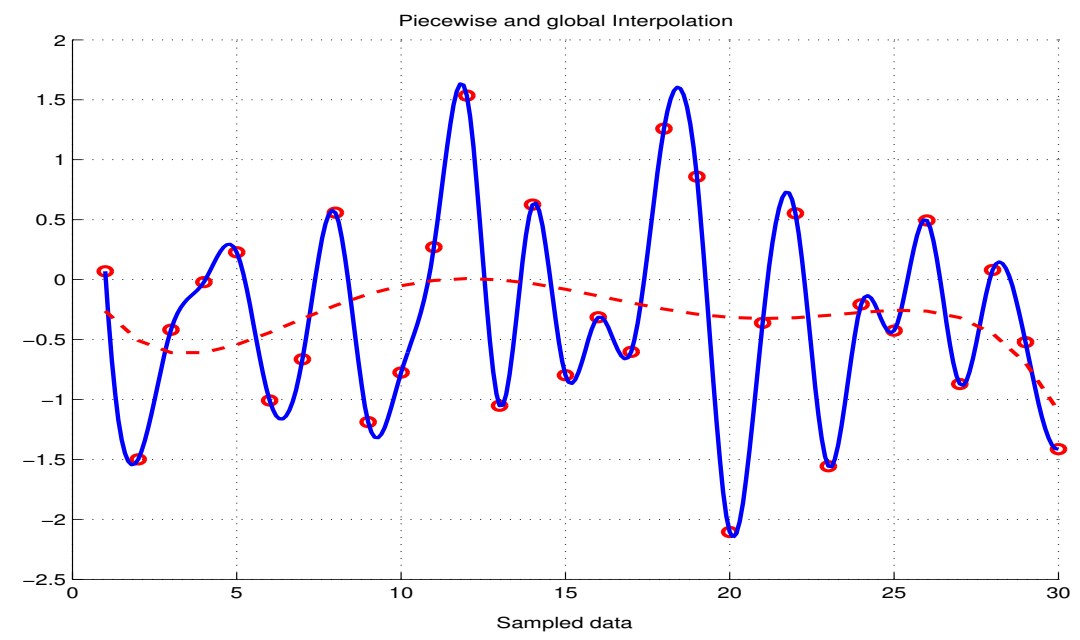

Figure 3.3: Global and piecewise interpolation

independent variable. First step is to construct a finite number of sampled data with uniform length for each independent variable. These sampled data are called $X_{i}$. Knot sequences are given as

$$
\text { Knot sequences }=\{0,0.2,0.4,0.6,0.8,1,1.2,1.4,1.6,1.8,2\}
$$

The next step is to apply augmented knot sequence to these set of data. From definition, augmented knot returns a nondecreasing knot sequence that has multiplicity $k$ for the first and last knot [3]. Knot multiplicity is 3, with multiplicity for first and last breaking points.

$$
\text { Augmented knot sequence }=\{0,0,0,0.2,0.4,0.6,0.8,1,1.2,1.4,1.6,1.8,2,2,2\}
$$

Apply moving average method to each set of data. Average of successive $k-1$ knots is defined as

$$
X_{i}^{*}=\frac{X_{i+1}+\ldots+X_{i+k-1}}{k-1}
$$

With knot sequence defined as

$$
X=\left(X_{i}^{*}\right)_{i=1}^{n+k}
$$




$$
\begin{gathered}
X_{1}^{*}=\frac{X_{2}+X_{3}}{2}=0 \\
X_{2}^{*}=\frac{X_{3}+X_{4}}{2}=0.1 \\
X_{3}^{*}=\frac{X_{4}+X_{5}}{2}=0.3 \\
X_{4}^{*}=\frac{X_{5}+X_{6}}{2}=0.5 \\
X_{5}^{*}=\frac{X_{6}+X_{7}}{2}=0.7 \\
X_{6}^{*}=\frac{X_{7}+X_{8}}{2}=0.9 \\
X_{7}^{*}=\frac{X_{8}+X_{9}}{2}=1.1 \\
X_{8}^{*}=\frac{X_{9}+X_{10}}{2}=1.3 \\
X_{9}^{*}=\frac{X_{10}+X_{11}}{2}=1.5 \\
X_{12}^{*}=\frac{X_{13}+X_{14}}{2}=2 \\
X_{10}^{*}=\frac{X_{11}+X_{12}}{2}=1.7 \\
X_{12}+X_{13} \\
2.9
\end{gathered}
$$

Where $n$ is the number of data in this case $n=12$. Knot averages method is recommended for a better spline approximation of order $k$ with knot sequence $X$. Knot averages values for augmented knot sequence with previous set of data are computed. The final result is given as

$$
\text { Knot averages }=\{0,0.1,0.3,0.5,0.7,0.9,1.1,1.3,1.5,1.7,1.9,2\}
$$

So the cubic spline interpolation is applied to new set of data which is given by the knot average method. Spline approach is especially attractive in the characterization and calculation of 
optimal solution. As a result, it gives proper evaluation of mathematical function with low order polynomial which can replace the original function. The basic idea of approximation theory is to resolve the complicated function, called target function, into simpler functions or approximants. So in approximation theory one assumes that sampled values from original function are available. The information is then used to construct the approximants with spline interpolation method which is in form of piecewise polynomial with low order.

\subsection{Cubic Spline}

In this part, mathematical proof for cubic spline is given in detail [14]. Define following equalities

$$
\begin{aligned}
h_{i} & =\tau_{i+1}-\tau_{i} \\
d_{i} & =\frac{z_{i+1}-z_{i}}{\tau_{i+1}-\tau_{i}} \\
s_{i} & =z^{\prime \prime}\left(\tau_{i}\right)
\end{aligned}
$$

This notation implies that $z(\tau)$ is piecewise cubic. Also $z^{\prime}(\tau)$ is piecewise quadratic and $z^{\prime \prime}(\tau)$ is piecewise linear. Performing Lagrange interpolation or piecewise linear interpolation for the set of data yields

$$
z_{i}^{\prime \prime}(\tau)=z^{\prime \prime}\left(\tau_{i}\right) \frac{\tau-\tau_{i+1}}{\tau_{i}-\tau_{i+1}}+z^{\prime \prime}\left(\tau_{i+1}\right) \frac{\tau-\tau_{i}}{\tau_{i+1}-\tau i}
$$

Substituting equations (3.27) and (3.29) in (3.30).

$$
z_{i}^{\prime \prime}(\tau)=\frac{s_{i}\left(\tau_{i+1}-\tau\right)}{h_{i}}+\frac{s_{i+1}\left(\tau-\tau_{i}\right)}{h_{i}}
$$

Where $i=0,1,2, \ldots, n-1$ and $\tau \in\left[\tau_{i}, \tau_{i+1}\right]$. Obtaining the first derivative and the interpolant by integrating (3.31). Note that $p$ and $q$ are considered as constant values.

$$
\begin{array}{r}
z_{i}^{\prime}(\tau)=-\frac{s_{i}\left(\tau_{i+1}-\tau\right)^{2}}{2 h_{i}}+\frac{s_{i+1}\left(\tau-\tau_{i}\right)^{2}}{2 h_{i}}-p_{i}+q_{i} \\
z_{i}(\tau)=\frac{s_{i}\left(\tau_{i+1}-\tau\right)^{3}}{6 h_{i}}+\frac{s_{i+1}\left(\tau-\tau_{i}\right)^{3}}{6 h_{i}}+p_{i}\left(\tau_{i+1}-\tau\right)+q_{i}\left(\tau-\tau_{i}\right)
\end{array}
$$


In order to obtain $p$ and $q$, the interpolant needs to be evaluated at $\tau=\tau_{i}, \tau=\tau_{i+1}$. It requires also that $z_{i}\left(\tau_{i}\right)=z_{i}$ and $z_{i+1}\left(\tau_{i+1}\right)=z_{i+1}$ which are the values of polynomials at specific points.

$$
\begin{array}{r}
z_{i}\left(\tau_{i}\right)=\frac{s_{i}\left(\tau_{i+1}-\tau_{i}\right)^{3}}{6 h_{i}}+p_{i}\left(\tau_{i+1}-\tau_{i}\right) \Longrightarrow p_{i}=\frac{z_{i}}{h_{i}}-\frac{s_{i} h_{i}}{6} \\
z_{i}\left(\tau_{i+1}\right)=\frac{s_{i+1}\left(\tau_{i+1}-\tau_{i}\right)^{3}}{6 h_{i}}+q_{i}\left(\tau_{i+1}-\tau_{i}\right) \Longrightarrow q_{i}=\frac{z_{i+1}}{h_{i}}-\frac{s_{i+1} h_{i}}{6}
\end{array}
$$

Substituting $p, q$ into 3.33

$$
z_{i}(\tau)=\frac{s_{i}\left(\tau_{i+1}-\tau\right)^{3}}{6 h_{i}}+\frac{s_{i+1}\left(\tau-\tau_{i}\right)^{3}}{6 h_{i}}+\left(\frac{z_{i}}{h_{i}}-\frac{s_{i} h_{i}}{6}\right)\left(\tau_{i+1}-\tau\right)+\left(\frac{z_{i+1}}{h_{i}}-\frac{s_{i+1} h_{i}}{6}\right)\left(\tau-\tau_{i}\right)
$$

Continuity of first derivative shows that

$$
z_{i-1}^{\prime}\left(\tau_{i}\right)=z_{i}^{\prime}\left(\tau_{i}\right)
$$

Using 3.37 in 3.32 yields into

$$
\begin{array}{r}
z_{i}^{\prime}(\tau)=-\frac{s_{i}\left(\tau_{i+1}-\tau\right)^{2}}{2 h_{i}}+\frac{s_{i+1}\left(\tau-\tau_{i}\right)^{2}}{2 h_{i}}-\left(\frac{z_{i}}{h_{i}}-\frac{s_{i} h_{i}}{6}\right)+\left(\frac{z_{i+1}}{h_{i}}-\frac{s_{i+1} h_{i}}{6}\right) \\
z_{i-1}^{\prime}(\tau)=-\frac{s_{i-1}\left(\tau_{i}-\tau\right)^{2}}{2 h_{i-1}}+\frac{s_{i}\left(\tau-\tau_{i-1}\right)^{2}}{2 h_{i-1}}-\left(\frac{z_{i-1}}{h_{i-1}}-\frac{s_{i-1} h_{i-1}}{6}\right)+\left(\frac{z_{i}}{h_{i-1}}-\frac{s_{i} h_{i-1}}{6}\right)
\end{array}
$$

Evaluating (3.38) and (3.39) for $\tau=\tau_{i}$ and $\tau=\tau_{i-1}$ gives

$$
\begin{array}{r}
z_{i}^{\prime}\left(\tau_{i}\right)=-\frac{s_{i}\left(\tau_{i+1}-\tau_{i}\right)^{2}}{2 h_{i}}-\left(\frac{z_{i}}{h_{i}}-\frac{s_{i} h_{i}}{6}\right)+\left(\frac{z_{i+1}}{h_{i}}-\frac{s_{i+1} h_{i}}{6}\right) \\
z_{i-1}^{\prime}\left(\tau_{i}\right)=\frac{s_{i}\left(\tau_{i}-\tau_{i-1}\right)^{2}}{2 h_{i-1}}-\left(\frac{z_{i-1}}{h_{i-1}}-\frac{s_{i-1} h_{i-1}}{6}\right)+\left(\frac{z_{i}}{h_{i-1}}-\frac{s_{i} h_{i-1}}{6}\right)
\end{array}
$$

Applying the continuity of first derivative conditions results in

$$
-\frac{s_{i} h_{i}}{2}-\frac{z_{i}}{h_{i}}+\frac{s_{i} h_{i}}{6}+\frac{z_{i+1}}{h_{i}}-\frac{s_{i+1} h_{i}}{6}=\frac{s_{i} h_{i-1}}{2}-\frac{z_{i-1}}{h_{i-1}}+\frac{s_{i-1} h_{i-1}}{6}+\frac{z_{i}}{h_{i-1}}-\frac{s_{i} h_{i-1}}{6}
$$

By simplifying both sides of the equation, the difference equation for the cubic spline is obtained

$$
h_{i-1} s_{i-1}+\left(2 h_{i-1}+2 h_{i}\right) s_{i}+h_{i} s_{i+1}=6\left(\frac{z_{i+1}-z_{i}}{h_{i}}-\frac{z_{i}-z_{i-1}}{h_{i-1}}\right)
$$


Substitute the equalities given by (3.28)

$$
\begin{aligned}
d_{i} & =\frac{z_{i+1}-z_{i}}{\tau_{i+1}-\tau_{i}} \\
d_{i-1} & =\frac{z_{i}-z_{i-1}}{\tau_{i}-\tau_{i-1}}
\end{aligned}
$$

Allows 3.42 to become

$$
h_{i-1} s_{i-1}+\left(2 h_{i-1}+2 h_{i}\right) s_{i}+h_{i} s_{i+1}=6\left(d_{i}-d_{i-1}\right)
$$

Once the end conditions are specified, the spline coefficients can be determined uniquely by fixed solving the set of linear equations for $s$ and then solving for the coefficients of polynomial as given by (3.2) - 3.5). As a result spline function can be constructed by using polynomial coefficients for each segment. 


\section{Chapter 4}

\section{Applications}

\subsection{Tracking Controller Design for Duffing Oscillator}

In this case study, the objective is to drive the state of the system into the desired path. This task is fulfilled with help of exact feedback linearization method which is applied to forced Duffing oscillator. Consider the forced Duffing oscillator as formulated in (2.6) with the following parameters $q=0.31, p=0.2, p_{1}=-1, p_{2}=0.05, p_{3}=1$, and $\omega=1$. The resulting differential equation contains quadratic and cubic nonlinearities.

$$
\ddot{x}+0.2 \dot{x}-x+0.05 x^{2}+x^{3}=0.31 \cos (t)+u
$$

Following the control law as mentioned in (2.28), $u$ is given by

$$
u=0.2 x_{2}-x_{1}+0.05 x_{1}^{2}+x_{1}^{3}-0.31 \cos (t)-\sin (t)-C_{0}\left(x_{1}-\sin (t)\right)-C_{1}\left(x_{2}-\cos (t)\right)
$$

Pole placement method is used for tuning the coefficients $C_{0}, C_{1}$. The numerical values are obtained as $C_{0}=3$ and $C_{1}=1.5$. State trajectory $x_{1}$ for the system of Duffing oscillator is shown without any controller input in Figure 4.1. The controller needs to act in a way that brings the state trajectory to the desired path. Desired path is considered as periodic sine wave. The controller signal is applied at $t=38$ seconds. Figure 4.2 and Figure 4.3 show the state trajectory and controller signal, respectively.

The spline function as mentioned in $(4.2)$ is used to approximate the nonlinear controller. 


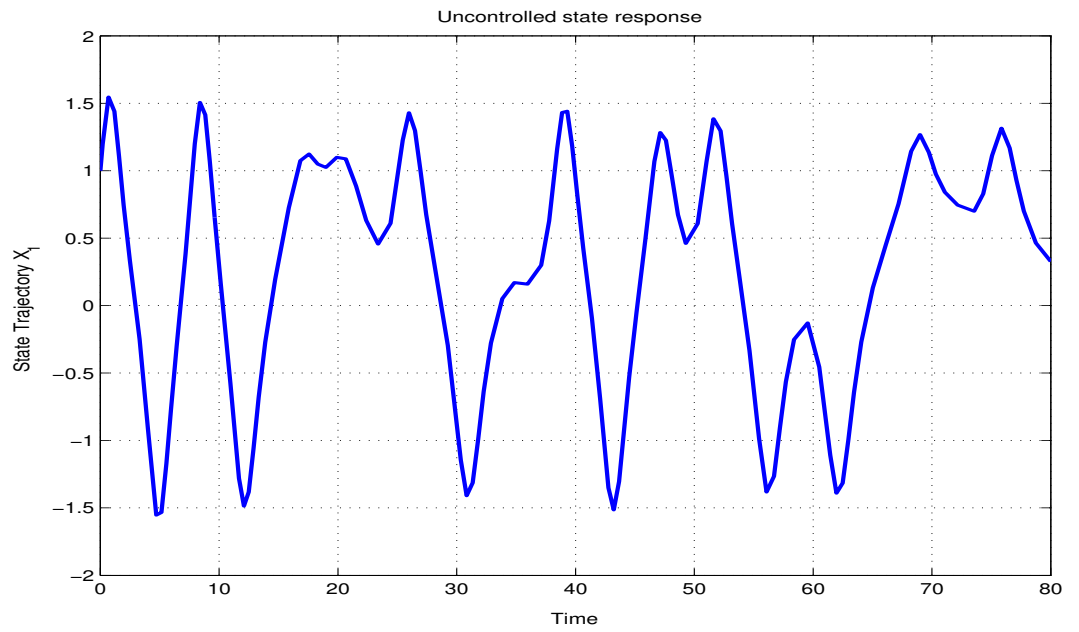

Figure 4.1: Uncontrolled response

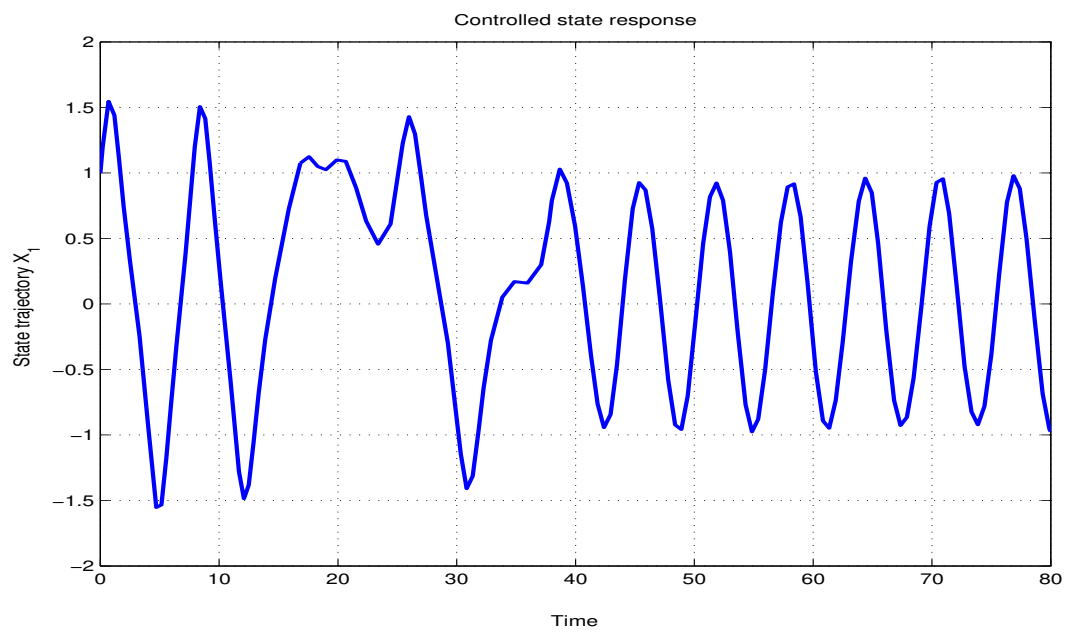

Figure 4.2: Controlled response

Generally, the equation can be written in form of

$$
u=\text { Nonlinear Function }\left(x_{1}, x_{2}, x_{3}\right)=F\left(x_{1}, x_{2}, x_{3}\right)
$$

The procedure for constructing the controller based in splines is explained in the following

Consider a limited number of sampled data points for $x_{1}, x_{2}$, and $x_{3}$ which are equally spaced and in an increasing sequence. Applying augmented knot sequence to these set of data. As mentioned in section 3.3 , augmented knot returns a nondecreasing knot sequence that has multiplicity of $k=3$ for the first and last knot. Output sampled data from controller is obtained with new 


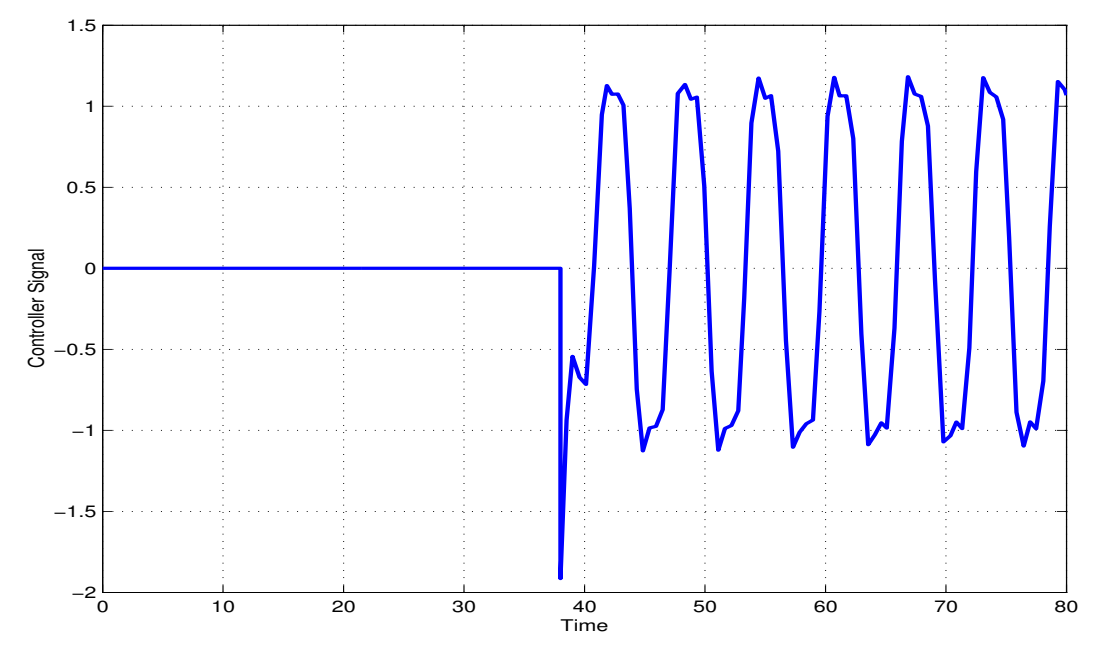

Figure 4.3: Controller signal

set of knots. Spline function is constructed for these set of input-output data in form of piecewise polynomials.

A piecewise polynomial is a function defined on an interval $[a, b]$ that is divided into subintervals $\left[x_{i}, x_{i+1}\right]$, where $i=0,1,2, \ldots, n-1$ with end point conditions as $x_{0}=a$ and $x_{n}=b$. On each subinterval, $\left[x_{i}, x_{i+1}\right]$, the function is equal to a polynomial which is called $P_{i}(x)$. Piecewise polynomial spline of order $k$ provides a definition in terms of its breaks $x_{1}, x_{2}, x_{3}, \ldots, x_{l+1}$ and general local polynomial coefficients $\xi_{i j}$ of its breaking pieces. General formulation for these set of polynomials is

$$
P_{i}(x)=\sum_{j=1}^{k}\left(x-x_{i}\right)^{k-j} \xi_{i j}
$$

A cubic spline polynomial can be written as follow.

$$
P_{i}(x)=\xi_{i 1}\left(x-x_{1}\right)^{3}+\xi_{i 2}\left(x-x_{2}\right)^{2}+\xi_{i 3}\left(x-x_{3}\right)+\xi_{i 4}
$$

Different polynomial order can be used for approximation process. Schematic view of conventional EFL controller and spline approximation based are shown in Figure 4.4. Depending on degree of polynomial, different controller path is approximated with spline function. The complexity of the controller is increased by increasing the polynomial coefficients between each segment of 


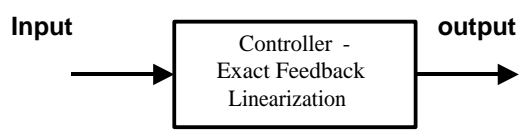

a)

b)

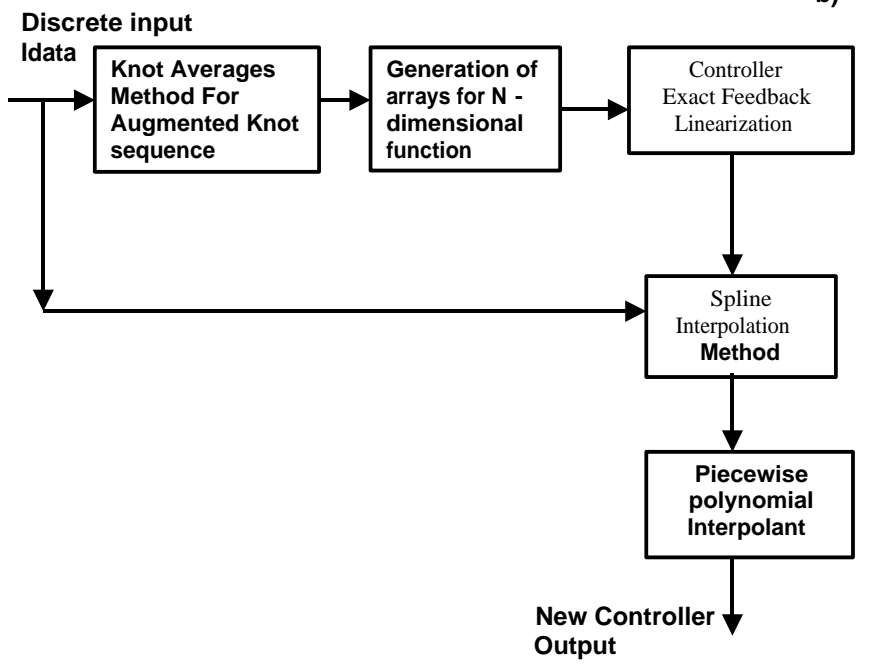

Figure 4.4: (a)-EFL controller (b)-spline controller

points. Figure 4.5 shows controller signals with different polynomial degree. State trajectories for different order of polynomials are shown in Figure 4.6. Desired path is taken as periodic sine function.

It is possible to estimate the difference between the given function $f(x)$ and its complete cubic spline interpolant, with bounds involving the maximum absolute values of the higher order derivatives of $f(x)$. Basic definitions for cubic spline end conditions interpolation is given as follow

Clamped Spline $-S^{\prime}(a)=f^{\prime}(a)$ and $S^{\prime}(b)=f^{\prime}(b)$

Natural Spline $-S^{\prime \prime}(a)=S^{\prime \prime}(b)=0$

Not a Knot condition $-S^{\prime \prime \prime}$ is continuous at $x_{1}$ and $x_{n-1}$.

Suppose that $f$ and its first four derivatives are continuous on interval $[a, b]$. Consider the standard notation

$$
M_{k}=\max \left|f^{(k)}(x)\right|: x_{0} \leq x \leq x_{n}
$$

Defining the approximated spline function $S(x)$ which construct the new approximants on 

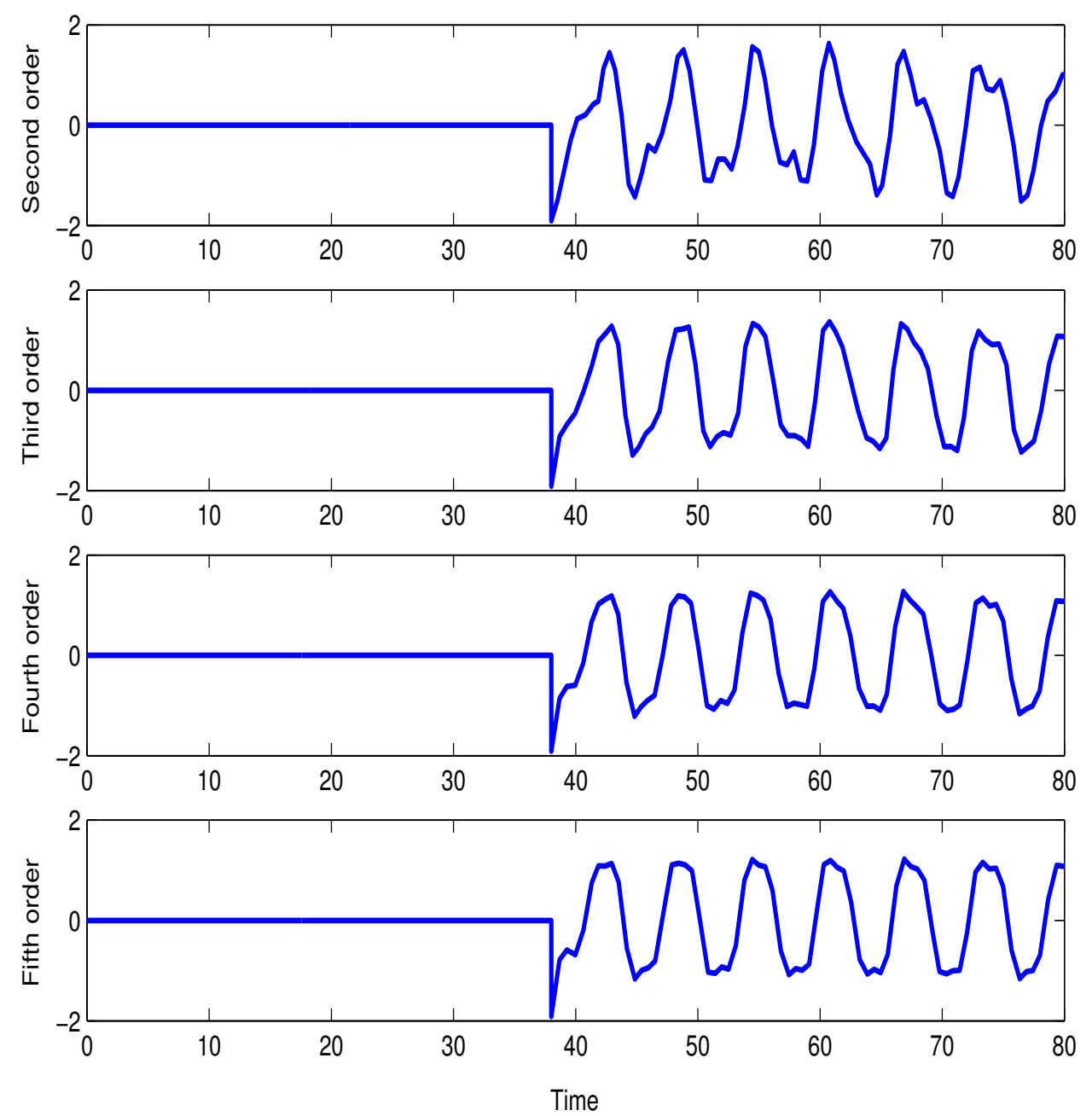

Figure 4.5: Controller signal

points $x_{k}$ for $k=0,1,2, \ldots, n$ with $a=x_{0}<x_{1}<\ldots<x_{n-1}<x_{n}=b$. Burden et al. [5] presented the error bound for spline interpolation and original function

$$
\max _{a \leq x \leq b}|f(x)-S(x)| \leq \frac{5 M_{4}}{384} \max _{0 \leq j \leq n-1}\left(x_{j+1}-x_{j}\right)^{4}
$$

The error bound is valid for various end conditions of cubic spline such as clamped spline, natural spline, and Not a knot condition. Spline approach is attractive in characterization and calculation of the optimal solution and has been used previously, for instance in Netravali et al. [25]. Their work is based on the relationship between a class of minimum energy control problems and spline interpolation technique. The system that has been investigated includes dynamical interconnected systems and it has number of single-input single-output finite dimensional linear 

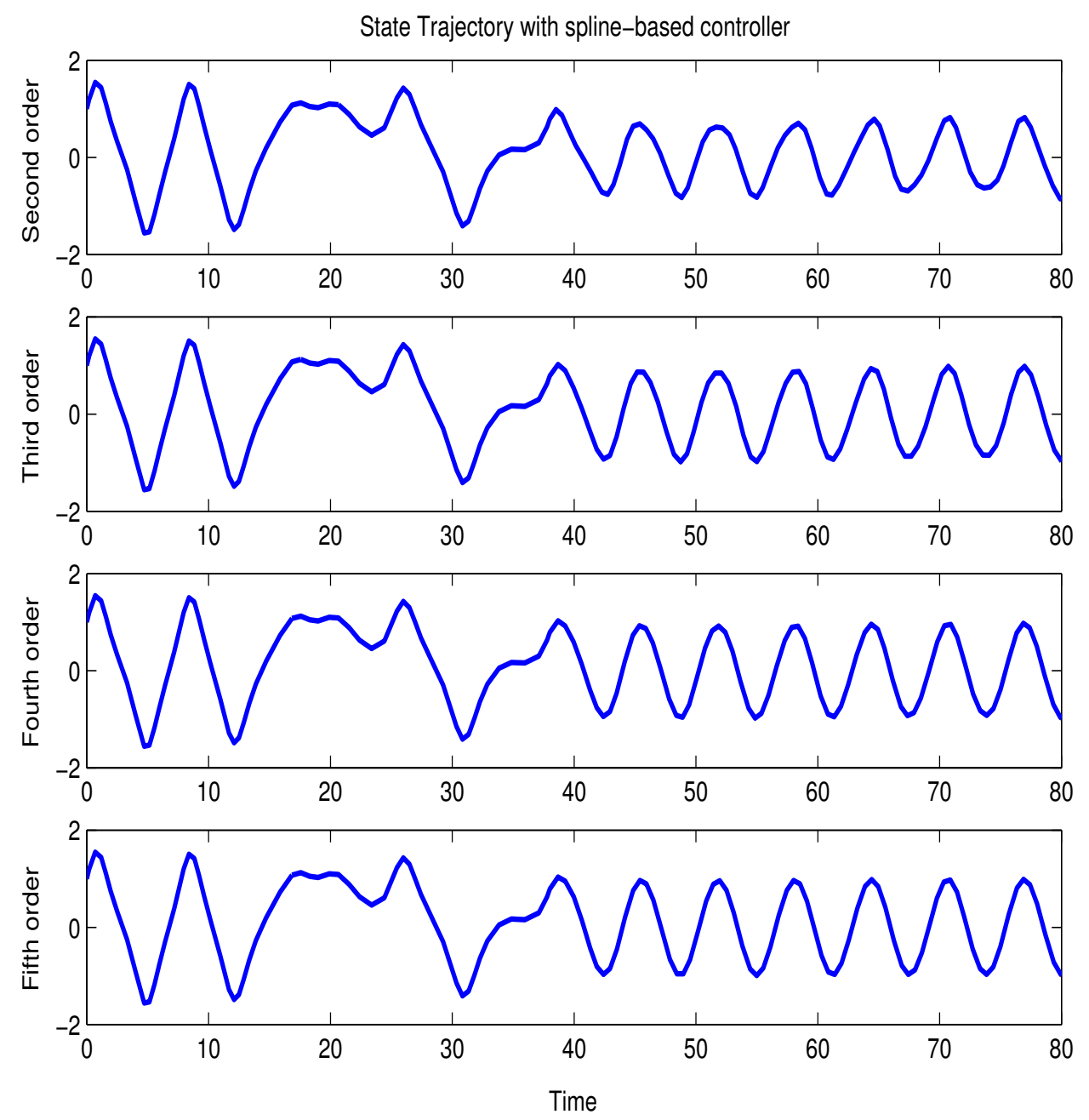

Figure 4.6: State trajectories for different polynomial degree

time varying dynamical subsystems.

Spline method is used to approximate nonlinear controller based on EFL controller for tracking problem in a way that controller effort is reduced. Controller effort is defined as

$$
\begin{aligned}
& E_{1}=\int_{0}^{t} u(t)^{2} d t \\
& E_{2}=\int_{0}^{t} t u(t)^{2} d t
\end{aligned}
$$

On each input-output set of vector, polynomial is interpolated with different order. Table 4.1 
shows that for different combination of piecewise polynomial, the controller effort varies.

In comparison with spline based controller effort, EFL controller needs larger amount of energy for driving the state of the system into desired path. For instance, controller effort with EFL method is $E_{1}=0.411, E_{2}=10.505$ whereas the cubic spline based has $E_{1 s}=0.405$, $E_{2 s}=10.417$. This study can also be done in frequency domain by computing power spectral density (PSD). In the frequency domain, PSD shows distribution of power per unit frequency $(\mathrm{Hz})$. For two controller signals, EFL and spline-based, the average power of spectral density are compared with each other. As expected from previous analysis, results show that with splinebased controller distribution of power per unit frequency is much less than the original EFL controller. Note that real value of sampled data produce a one-sided power spectral density. This method is called periodogram. Mathematical formula is given by

$$
P_{x x}(f)=\frac{\left|U_{L}(f)\right|^{2}}{f_{s} L}
$$

where

$$
U_{L}(f)=\sum_{n=0}^{L} U_{L}[n] e^{\frac{-2 f_{n} \pi j}{f_{s}}}
$$

The parameters are

$$
\begin{array}{lll}
L & \ldots & \text { length of the sampled data } \\
U_{L}[n] & \ldots & \text { sampled data } \\
f_{s} & \ldots & \text { sampling frequency } \\
P_{x x}(f) & \ldots & \text { power spectral density }
\end{array}
$$

Periodogram is a method in which the estimate of the PSD is made from the signal directly. The spectral density is computed in units of power per hertz. Polynomials with different orders for controller are constructed. It shows power contained in the sampled data from the splinebased controller signals has less density of power through the frequency range while satisfying the trajectory of the desired path. For example the PSD for controller signal with exact feedback linearization method is 3.1729 , and with cubic spline is 3.1002 . Table 4.2 shows PSD for spline based controller with different order of polynomial between each set of input-output data. Periodogram between DFL controller and cubic spline controller is shown in Figure 4.7.

Spline method was used to approximate the nonlinear EFL controller. Controller effort in spline-based with different order, showed better performance in time domain when compared 


\begin{tabular}{|c|c|c|c|c|}
\hline $\begin{array}{l}\text { Piecewise } \\
\text { polynomial } \\
\text { order } \\
\text { (First input) } \\
\mathrm{X}_{1}\end{array}$ & \begin{tabular}{|l} 
Piecewise \\
polynomial \\
order \\
(Second \\
input) \\
$\mathrm{X}_{2}$ \\
\end{tabular} & \begin{tabular}{|l} 
Piecewise \\
polynomial \\
order \\
(Third input) \\
$\mathrm{X}_{3}$
\end{tabular} & $\begin{array}{l}\text { Controller } \\
\text { effort } \\
\mathrm{E}_{1 \mathrm{~S}} \quad 10^{3}\end{array}$ & $\begin{array}{l}\text { Controller } \\
\text { effort } \\
\mathrm{E}_{2 \mathrm{~S}} \quad 10^{3}\end{array}$ \\
\hline 1 & 1 & 1 & 0.868 & 38.267 \\
\hline 1 & 1 & 3 & 0.550 & 19.097 \\
\hline 1 & 1 & 5 & 0.544 & 18.538 \\
\hline 1 & 3 & 1 & 0.768 & 31.637 \\
\hline 1 & 3 & 3 & 0.472 & 14.793 \\
\hline 1 & 3 & 5 & 0.484 & 15.345 \\
\hline 1 & 5 & 1 & 0.768 & 31.637 \\
\hline 1 & 5 & 3 & 0.472 & 14.793 \\
\hline 1 & 5 & 5 & 0.484 & 15.345 \\
\hline 3 & 1 & 1 & 0.759 & 30.334 \\
\hline 3 & 1 & 3 & 0.485 & 15.023 \\
\hline 3 & 1 & 5 & 0.490 & 15.042 \\
\hline 3 & 3 & 1 & 0.637 & 23.262 \\
\hline 3 & 3 & 3 & 0.403 & 10.412 \\
\hline 3 & 3 & 5 & 0.408 & 10.408 \\
\hline 3 & 5 & 1 & 0.637 & 23.262 \\
\hline 3 & 5 & 3 & 0.403 & 10.412 \\
\hline 3 & 5 & 5 & 0.408 & 10.408 \\
\hline 5 & 1 & 1 & 0.754 & 30.050 \\
\hline 5 & 1 & 3 & 0.492 & 15.431 \\
\hline 5 & 1 & 5 & 0.493 & 15.241 \\
\hline 5 & 3 & 1 & 0.665 & 25.019 \\
\hline 5 & 3 & 3 & 0.403 & 10.413 \\
\hline 5 & 3 & 5 & 0.409 & 10.499 \\
\hline 5 & 5 & 1 & 0.665 & 25.019 \\
\hline 5 & 5 & 3 & 0.403 & 10.413 \\
\hline 5 & 5 & 5 & 0.409 & 10.499 \\
\hline
\end{tabular}

Table 4.1: Controller effort with spline-based controller 


\begin{tabular}{|c|c|c|c|}
\hline $\begin{array}{l}\text { Piecewise } \\
\text { polynomial } \\
\text { order } \\
\text { (First input) } \\
X_{1}\end{array}$ & \begin{tabular}{|l} 
Piecewise \\
polynomial \\
order \\
(Second input) \\
$\mathrm{X}_{2}$
\end{tabular} & \begin{tabular}{|l} 
Piecewise \\
polynomial \\
order \\
(Third input) \\
$\mathrm{X}_{3}$
\end{tabular} & $\begin{array}{l}\text { Average of } \\
\text { power spectral } \\
\text { density for } \\
\text { Spline } \\
\text { controller }\end{array}$ \\
\hline 1 & 1 & 1 & 2.7858 \\
\hline 1 & 1 & 3 & 1.7391 \\
\hline 1 & 1 & 5 & 1.7897 \\
\hline 1 & 3 & 1 & 2.9561 \\
\hline 1 & 3 & 3 & 1.9685 \\
\hline 1 & 3 & 5 & 1.9069 \\
\hline 1 & 5 & 1 & 2.9561 \\
\hline 1 & 5 & 3 & 1.9685 \\
\hline 1 & 5 & 5 & 1.9069 \\
\hline 3 & 1 & 1 & 3.0886 \\
\hline 3 & 1 & 3 & 2.2780 \\
\hline 3 & 1 & 5 & 2.3088 \\
\hline 3 & 3 & 1 & 3.5626 \\
\hline 3 & 3 & 3 & 3.0625 \\
\hline 3 & 3 & 5 & 3.1452 \\
\hline 3 & 5 & 1 & 3.5626 \\
\hline 3 & 5 & 3 & 3.0625 \\
\hline 3 & 5 & 5 & 3.1452 \\
\hline 5 & 1 & 1 & 2.9270 \\
\hline 5 & 1 & 3 & 2.1597 \\
\hline 5 & 1 & 5 & 2.2794 \\
\hline 5 & 3 & 1 & 3.6365 \\
\hline 5 & 3 & 3 & 3.0628 \\
\hline 5 & 3 & 5 & 3.1302 \\
\hline 5 & 5 & 1 & 3.6365 \\
\hline 5 & 5 & 3 & 3.0628 \\
\hline 5 & 5 & 5 & 3.1302 \\
\hline
\end{tabular}

Table 4.2: Power spectral density with spline-based controller 


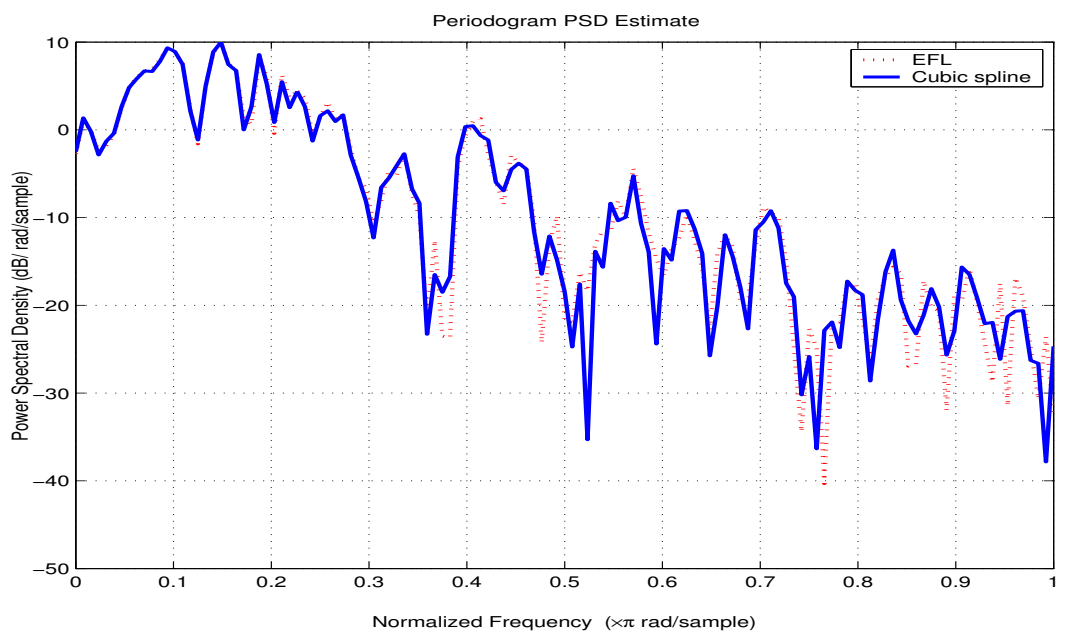

Figure 4.7: Periodogram between DFL and cubic spline

to the EFL. In frequency domain, average of power spectral density of the controller signal is compared with exact feedback linearizaton. Spline-based controller has an advantage of selecting the polynomial order within specific range while decreasing the controller effort. The order of piecewise interpolation can be chosen as linear, quadratic, cubic and also higher between a set of input-output data. 


\subsection{Excitation control with Direct Feedback Linearization Method}

Direct Feedback Linearization is a nonlinear control design tool which is implemented in two steps [30]. The first step is to obtain a linear system using feedback to compensate for the nonlinearities. The compensator can explicitly cancel the nonlinearities present in the machine. The second step is to design a controller using the feedback linearized system. The advantage of linear closed loop dynamics is that the selection of controller gains are simplified and classical controller designed can be used. By using DFL compensating law the multi-machine power system can be linearized and decoupled as state space equations.

One-axis model generator has been used previously for implementing DFL technique. The applicability of the method is based on type of nonlinearities. DFL controller will be used for two axis model generator. Controller design for excitation loop with DFL method concept is explained in two stages. First a brief description is given of the two-axis model generator. Consider the $i^{\text {th }}$ generator in multi machine power system. Two-axis model contains the set of differential and algebraic equations as follows

a) Differential equations

$$
\begin{aligned}
\dot{\delta}_{i}(t) & =\omega_{i}(t)-\omega_{0} \\
\dot{\omega}_{i}(t) & =\frac{-D_{i}}{2 H_{i}}\left(\omega_{i}(t)-\omega_{0}\right)+\frac{\omega_{0}}{2 H_{i}}\left(P_{m i}-P_{e i}\right) \\
\dot{E}_{d i}^{\prime} & =\frac{1}{T_{q o i}^{\prime}}\left[I_{q i}\left(X_{q i}-X_{q i}^{\prime}\right)-E_{d i}^{\prime}\right] \\
\dot{E}_{q i}^{\prime} & =\frac{1}{T_{d o i}^{\prime}}\left[E_{f l d i}-I_{d i}\left(X_{d i}-X_{d i}^{\prime}\right)\right]
\end{aligned}
$$

b) Algebraic equations

$$
\begin{aligned}
P_{e i} & =E_{d i}^{\prime} I_{d i}+E_{q i}^{\prime} I_{q i} \\
E_{d i} & =E_{d i}^{\prime}-I_{d i} r_{a i}+I_{q i} X_{d i}^{\prime} \\
E_{q i} & =E_{q i}^{\prime}-I_{q i} r_{a i}+I_{d i} X_{d i}^{\prime} \\
I_{q i}(t) & =\sum_{j=1}^{n} E_{q j}^{\prime}\left(B_{i j} \sin \delta_{i j}(t)+G_{i j} \cos \delta_{i j}(t)\right) \\
I_{d i}(t) & =\sum_{j=1}^{n} E_{q j}^{\prime}\left(G_{i j} \sin \delta_{i j}(t)-B_{i j} \cos \delta_{i j}(t)\right)
\end{aligned}
$$


The variables $I_{d}$ and $I_{q}$ are internal variables. When these variables are used in 4.15), it will yield the generator's electric power output. Hence, an alternative model will be to use this active power as a state variable instead of the internal voltages $E_{d}^{\prime}$ and $E_{q}^{\prime}$. The state equation of the active power is obtained by taking the derivative of 4.15

$$
\dot{P}_{e i}=\dot{E}_{d i}^{\prime} I_{d i}+E_{d i}^{\prime} \dot{I}_{d i}+\dot{E}_{q i}^{\prime} I_{q i}+E_{q i}^{\prime} \dot{I}_{q i}
$$

By substituting the dynamic state equations $4.13,4.14$ of $E_{d}^{\prime}$ and $E_{q}^{\prime}$ into 4.20 one obtains

$$
\begin{aligned}
\dot{P_{e i}}= & \frac{1}{T_{q o i}^{\prime}}\left[I_{q i}\left(X_{q i}-X_{q i}^{\prime}\right)-E_{d i}^{\prime}\right] I_{d i}+E_{d i}^{\prime} \dot{I}_{d i}+ \\
& +\frac{1}{T_{d o i}^{\prime}}\left[E_{f l d i}-I_{d i}\left(X_{d i}-X_{d i}^{\prime}\right)\right] I_{q i}+E_{q i}^{\prime} \dot{I}_{q i}
\end{aligned}
$$

Equations (4.11) and (4.12) remain the same. To obtain the feedback linearized system, assume that the mechanical power $P_{m i}$ is kept constant equal to $P_{m i}^{0}$. The accelerating power is then given by

$$
\Delta P_{e i}=P_{e i}-P_{m i}^{0}
$$

and its dynamics are governed by the following state equation, obtained from (4.21)

$$
\begin{aligned}
\Delta \dot{P}_{e i}=\dot{P}_{e i}= & \frac{I_{q i} I_{d i}\left(X_{q i}-X_{q i}^{\prime}\right)}{T_{q o i}^{\prime}}-\frac{E_{d i}^{\prime} I_{d i}}{T_{q o i}^{\prime}}+E_{d i}^{\prime} \dot{I}_{d i}+ \\
& +\frac{E_{f l d i} I_{q i}}{T_{d o i}^{\prime}}-\frac{I_{q i} I_{d i}\left(X_{d i}-X_{d i}^{\prime}\right)}{T_{d o i}^{\prime}}+E_{q i}^{\prime} \dot{I}_{q i}
\end{aligned}
$$

Using algebraic equation for electric power 4.15

$$
E_{d i}^{\prime} I_{d i}=\Delta P_{e i}-E_{q i}^{\prime} I_{q i}+P_{m i}^{0}
$$

Substitute 4.24 into 4.23

$$
\begin{aligned}
\Delta \dot{P_{e i}}= & -\frac{\Delta P_{e i}}{T_{q o i}^{\prime}}+\frac{I_{q i} I_{d i}\left(X_{q i}-X_{q i}^{\prime}\right)}{T_{q o i}^{\prime}}+\frac{E_{q i}^{\prime} I_{q i}}{T_{q o i}^{\prime}}-\frac{P_{m i}^{0}}{T_{q o i}^{\prime}}+ \\
& +E_{d i}^{\prime} \dot{I_{d i}}+E_{q i}^{\prime} \dot{I_{q i}}+\frac{E_{f l d_{i}} I_{q i}}{T_{d o i}^{\prime}}-\frac{I_{q i} I_{d i}\left(X_{d i}-X_{d i}^{\prime}\right)}{T_{d o i}^{\prime}}
\end{aligned}
$$


This can be written as

$$
\Delta \dot{P}_{e i}=-\frac{\Delta P_{e i}}{T_{q o i}^{\prime}}+\frac{V_{f i}(t)}{T_{q o i}^{\prime}}
$$

where

$$
\begin{aligned}
V_{f i}(t) & =I_{q i} I_{d i}\left(X_{q i}-X_{q i}^{\prime}\right)+E_{q i}^{\prime} I_{q i}-P_{m i}^{0}+E_{d i}^{\prime} \dot{I}_{d i} T_{q o i}^{\prime}+ \\
& +E_{q i}^{\prime} \dot{I}_{q i} T_{q o i}^{\prime}+\frac{T_{q o i}^{\prime}}{T_{d o i}^{\prime}} I_{q} E_{f l d i}-\frac{T_{q o i}^{\prime}}{T_{d o i}^{\prime}} I_{d i} I_{q i}\left(X_{d i}-X_{d i}^{\prime}\right)
\end{aligned}
$$

Let the nominal values be $\delta_{i 0}, \omega_{i 0}$. Then define variations around these nominal values as

$$
\begin{array}{r}
\Delta \delta_{i}(t)=\delta_{i}(t)-\delta_{i 0} \\
\Delta \omega_{i}(t)=\omega_{i}(t)-\omega_{i 0}
\end{array}
$$

Their dynamics are given by

$$
\begin{aligned}
\Delta \dot{\delta}_{i} & =\Delta \omega_{i} \\
\Delta \dot{\omega}_{i} & =-\frac{D_{i}}{2 H_{i}} \Delta \omega_{i}-\frac{\omega_{i o}}{2 H_{i}} \Delta P_{e i}
\end{aligned}
$$

Equations 4.30, 4.31 and 4.26 form the feedback linearized model for the $i^{\text {th }}$ generator. This model is written in matrix form as

$$
\dot{x}_{i}(t)=A_{i} x_{i}+B_{i} V_{f i}(t)
$$

Where the state variables are $x_{i}^{T}=\left[\Delta \delta_{i}(t) \Delta \omega_{i}(t) \Delta P_{e i}(t)\right]$ and

$$
A_{i}=\left(\begin{array}{ccc}
0 & 1 & 0 \\
0 & -\frac{D_{i}}{2 H_{i}} & -\frac{\omega_{i 0}}{2 H_{i}} \\
0 & 0 & -\frac{1}{T_{q o i}^{\prime}}
\end{array}\right) B_{i}=\left(\begin{array}{c}
0 \\
0 \\
\frac{1}{T_{q o i}^{\prime}}
\end{array}\right)
$$

\subsubsection{Control Design}

Using the linearized system given by 4.32 the controller $V_{f i}$ can be designed via different methods such as pole placement. It is a linear state feedback controller of the form

$$
V_{f i}=-K_{i} x_{i}=-K_{\delta_{i}} \Delta \delta_{i}-K_{\omega_{i}} \Delta \omega_{i}-K_{P e i} \Delta P_{e i}
$$

where $K_{\delta i}, K_{\omega i}$, and $K_{P e i}$ are constant gains. 


\subsubsection{Nonlinear Controller}

Finally the nonlinear controller is obtained from (4.27) and 4.34

$$
\begin{array}{r}
E_{f l d_{i}}(t)=\frac{T_{d o i}^{\prime}}{T_{q o i}^{\prime} I_{q i}}\left[-K_{\delta_{i}} \Delta \delta_{i}-K_{\omega_{i}} \Delta \omega_{i}-K_{P e i} \Delta P_{e i}\right]+\left[-\frac{T_{d o i}^{\prime}}{T_{q o i}^{\prime}} I_{d i}\left(X_{q i}-X_{q i}^{\prime}\right)-\right. \\
\left.-\frac{E_{q i}^{\prime} T_{d o i}^{\prime}}{T_{q o i}^{\prime}}+\frac{P_{m i} T_{d o i}^{\prime}}{T_{q o i}^{\prime} I_{q i}}-\frac{E_{d i}^{\prime} \dot{I}_{d i} T_{d o i}^{\prime}}{I_{q i}}-\frac{E_{q i}^{\prime} \dot{I}_{q i} T_{d o i}^{\prime}}{I_{q i}}+I_{d i}\left(X_{d i}-X_{d i}^{\prime}\right)\right]
\end{array}
$$

From analysis above it is clear that when a compensating law is employed, the plant is linearized and the linearization is valid over a wide range of operating points. The controller includes linear state feedback loop plus nonlinear compensator and it is valid for a wide range except $I_{q}(t)=0$. The case where $I_{q}(t)=0$ is a singular point. After linearization, it is easy to implement deterministic method such as LQR [27] or even probabilistic method as genetic algorithm for tuning controller in achieving desired stability and performance properties. Spline technique is used to approximate the controller given in (4.35) with piecewise polynomials. 


\subsection{Three Machine Nine Bus Test System}

A three machine nine bus test system, Figure 4.8, as described by Anderson and Fouad [2] is used to illustrate the application of spline based controller in comparison with DFL controller. The parameters for generators are given in Table I. Note that the rotor angle and speed of generator number 1 is chosen as the reference. Three cases are considered, loss of line $5-7$, loss of load at bus 5 , and a line to ground fault on line $5-7$.

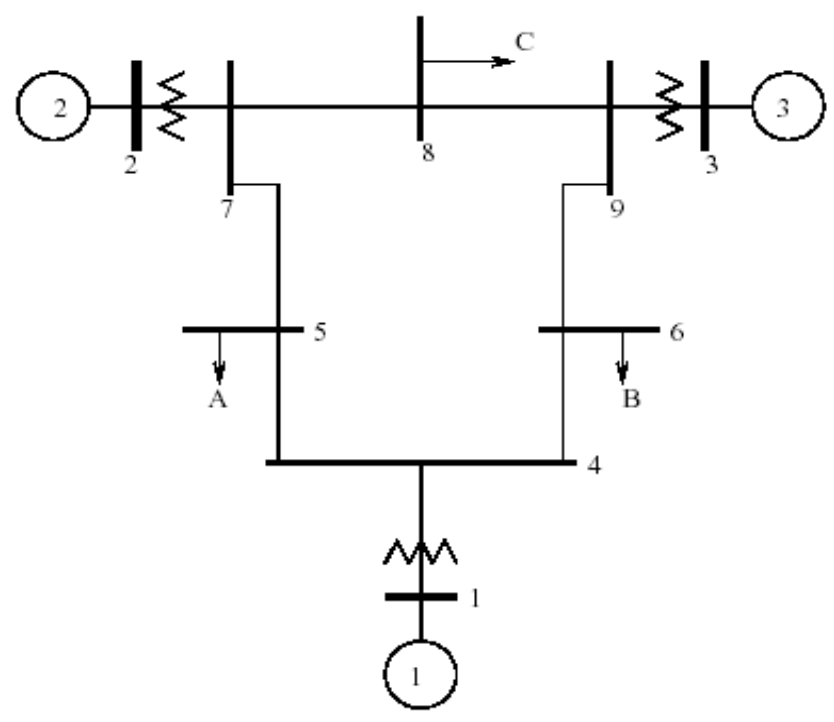

Figure 4.8: Three Machine Nine Bus Test System

Table 4.3: Generator parameters

\begin{tabular}{|c|c|c|c|c|c|c|c|}
\hline Machine & $X_{d i}$ & $X_{d i}^{\prime}$ & $T_{d o i}^{\prime}$ & $X_{q} i$ & $X_{q i}^{\prime}$ & $T_{q o i}^{\prime}$ & $H_{i}$ \\
\hline 1 & 0.146 & 0.0608 & 8.96 & 0.0969 & 0.0969 & 0.31 & 23.64 \\
\hline 2 & 0.8958 & 0.1198 & 6.00 & 0.8645 & 0.1969 & 0.535 & 6.40 \\
\hline 3 & 1.3125 & 0.1813 & 5.89 & 1.2578 & 0.2500 & 0.600 & 3.01 \\
\hline
\end{tabular}

When applying the (DFL), the linearized model is

$$
\dot{x_{i}}=A_{i} x_{i}(t)+B_{i} V_{f i}(t)
$$

For $\{i=1,2,3\} \quad A_{i}$ and $B_{i}$ are obtained 


$$
\begin{aligned}
& A_{1}=\left(\begin{array}{ccc}
0 & 1 & 0 \\
0 & -0.0212 & -0.0212 \\
0 & 0 & -3.2258
\end{array}\right) \quad B_{1}=\left(\begin{array}{c}
0 \\
0 \\
3.2258
\end{array}\right) \\
& A_{2}=\left(\begin{array}{ccc}
0 & 1 & 0 \\
0 & -0.0781 & -0.0781 \\
0 & 0 & -1.8692
\end{array}\right) \quad B_{2}=\left(\begin{array}{c}
0 \\
0 \\
1.8692
\end{array}\right) \\
& A_{3}=\left(\begin{array}{ccc}
0 & 1 & 0 \\
0 & -0.1661 & -0.1661 \\
0 & 0 & -1.6667
\end{array}\right) \quad B_{3}=\left(\begin{array}{c}
0 \\
0 \\
1.6667
\end{array}\right)
\end{aligned}
$$

The controller $V_{f i}(t)$ can be designed by using different linear control design method. Here, LQR method is used. Following gains are obtained

$$
\begin{aligned}
V_{f 1}(t) & =-\Delta \delta_{1}-10.4483 \Delta \omega_{1}+0.4619 \Delta P_{e 1} \\
V_{f 2}(t) & =-\Delta \delta_{2}-5.0708 \Delta \omega_{2}+0.5569 \Delta P_{e 2} \\
V_{f 3}(t) & =-\Delta \delta_{3}-3.2505 \Delta \omega_{3}+0.6272 \Delta P_{e 3}
\end{aligned}
$$

DFL nonlinear controller is obtained by substituting the following linear functions into 4.35).

$$
\begin{aligned}
E_{f l i d_{1}} & =\frac{-28.9 \Delta \delta_{1}-301.9571 \Delta \omega_{1}+13.3475 \Delta P_{e 1}}{I_{q 1}(t)}- \\
& -28.9032 E_{q 1}^{\prime}+\frac{20.7062}{I_{q 1}}-8.96 E_{d 1}^{\prime} \frac{\dot{I_{d 1}}}{I_{q 1}}-8.96 E_{q 1}^{\prime} \frac{\dot{I_{q 1}}}{I_{q 1}}+0.0852 I_{d 1} \\
E_{f l i d_{2}}= & \frac{-11.21 \Delta \delta_{2}-56.8437 \Delta \omega_{2}+6.2427 \Delta P_{e 2}}{I_{q 2}(t)}- \\
& -7.49 I_{d 2}-11.214 E_{q 2}^{\prime}+\frac{18.2803}{I_{q 2}}-6 E_{d 2}^{\prime} \frac{I_{d 2}}{I_{q 2}}-6 E_{q 2}^{\prime} \frac{\dot{I_{q 2}}}{I_{q 2}}+0.6562 I_{d 2} \\
E_{f l i d_{3}}= & \frac{-9.810 \Delta \delta_{3}-31.8870 \Delta \omega_{3}+6.1533 \Delta P_{e 3}}{I_{q 3}(t)}- \\
- & 9.8932 I_{d 3}-9.816 E_{q 3}^{\prime}+\frac{8.3441}{I_{q 3}}-5.89 E_{d 3}^{\prime} \frac{I_{d 3}}{I_{q 3}}-5.89 E_{q 3}^{\prime} \frac{\dot{I_{q 3}}}{I_{q 3}}+1.1312 I_{d 3}
\end{aligned}
$$


From construction of DFL controller, it is clear that each controller has two parts which include linear compensator and nonlinear compensator:

$$
\begin{gathered}
\text { Controller }=\text { Linear Compensator }\left(\Delta \delta_{i}, \Delta \omega_{i}, \Delta P_{e i}\right)+ \\
\text { Nonlinear Compensator }\left(E_{d i}^{\prime}, E_{q i}^{\prime}, I_{d i}, I_{q i}, \dot{I}_{d i}, \dot{I}_{q i}\right)
\end{gathered}
$$

Nonlinear approximation with spline technique is used to approximate both parts of this controller. Figure 4.9 shows schematic procedure for obtaining the spline based controller.

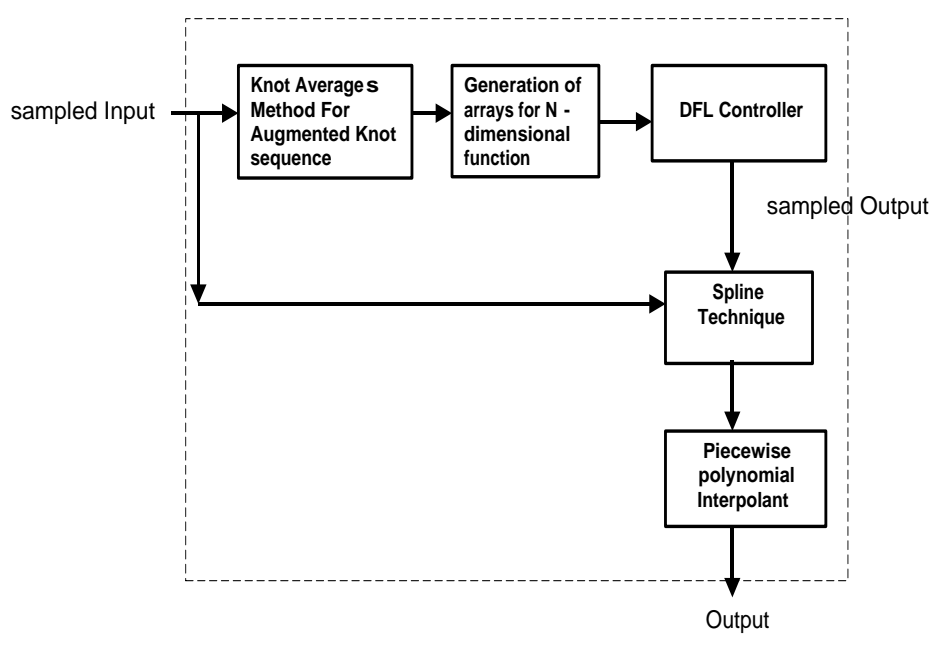

Figure 4.9: Schematic block diagram for spline-based controller

Usually spline is constructed from some information. These information can be function values, derivative values, or approximation solution to some ordinary differential equation. It is also possible to construct the spline function from scratch by providing its knot sequence and break sequence. In this case sampled input data includes equally spaced, increasing sequence with variation of independent variables of controller. These independent variables include $\delta_{i}, \omega_{i}, P_{e i}, E_{d i}^{\prime}, E_{q i}^{\prime}, I_{d i}, I_{q i}, \dot{I}_{d i}, \dot{I}_{q i}$. Knot averages method, as explained in section 3.3 is applied in order to obtain a new set of data. Combination of space for each three vectors is considered in multi-dimensional grid. Basically, this is the construction for the space in order to apply spline technique.

Cubic spline interpolation technique is applied through input-output set of data. The result is in form of piecewise polynomials. Dashed line in Figure 4.9 shows the spline based controller. Piecewise polynomial spline of order $k$ provides a definition in terms of its breaks $x_{1}, x_{2}, x_{3}, \ldots, x_{l+1}$ 
and general local polynomial coefficients $\xi_{i j}$ of its breaking pieces. General formulation for these set of polynomials is given as

$$
y_{i}=\sum_{j=1}^{k}\left(x-x_{i}\right)^{k-j} \xi_{i j}
$$

A cubic spline can be written as

$$
y_{i}=\xi_{i 1}\left(x-x_{1}\right)^{3}+\xi_{i 2}\left(x-x_{2}\right)^{2}+\xi_{i 3}\left(x-x_{3}\right)+\xi_{i 4}
$$

where coefficients $\xi_{i 1}, \xi_{i 2}, \xi_{i 3}$, and $\xi_{i 4}$ are equal to the coefficients 3.2 - 3.5.

\subsubsection{Case 1 - Loss of Line}

First contingency is a loss of line for a short period of time. The system is in steady state. Line $5-7$ is removed at 1 second and it is reconnected after 0.3 seconds. Constant field voltage is applied to each generator. Values for field voltage are computed from general load flow solution and are given by $E_{\text {filed } 1}=1.082, E_{\text {field } 2}=1.789$, and $E_{\text {field } 3}=1.402$. Here, the response of the system for constant type of field voltage is called open loop response. Next the proposed control strategy with spline technique is applied to the controller of each generator. Figure 4.10 , and Figure 4.11 compare relative speed and angle of machine 3 with respect to machine 1 for two cases of open loop response and closed loop response respectively. From the simulation it is clear that the closed loop system, with spline based nonlinear controller, stabilizes the disturbed system much faster than constant field voltages as expected.

\subsubsection{Case 2 - Loss of Load}

The load which is connected to bus 5 is removed for 0.13 seconds. Load $A$ form Figure 4.8 is given as $P_{L}=1.25$ p.u, $Q_{L}=0.5$ p.u. Figure 4.12 and Figure 4.13 compare relative speed and angle of machine 2 with respect to machine 1 for open and closed loop scenarios. 


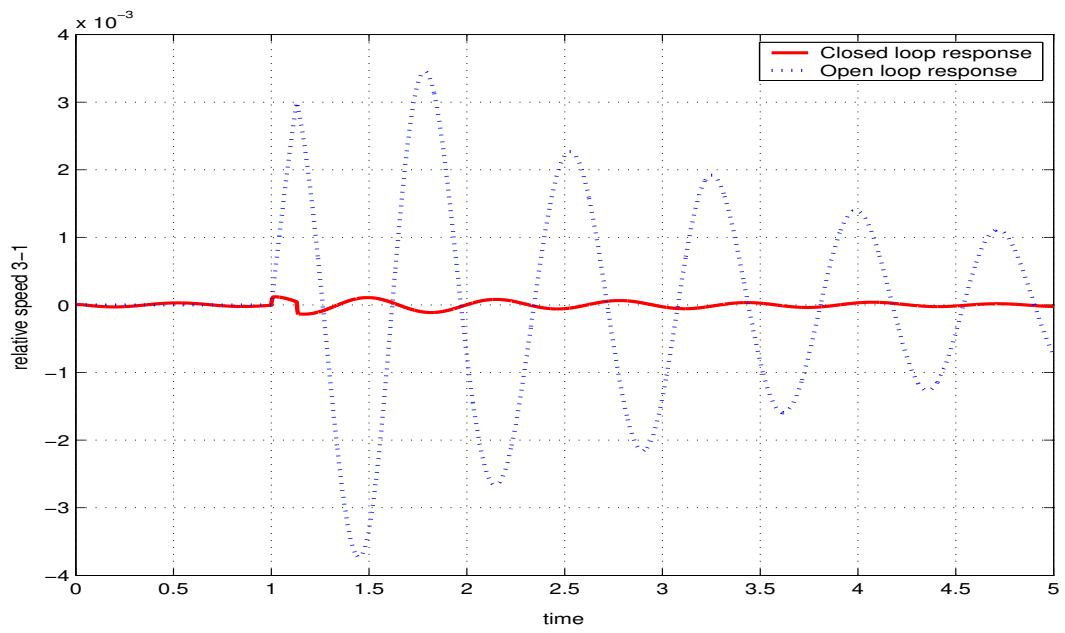

Figure 4.10: Case 1 Relative speed 3-1

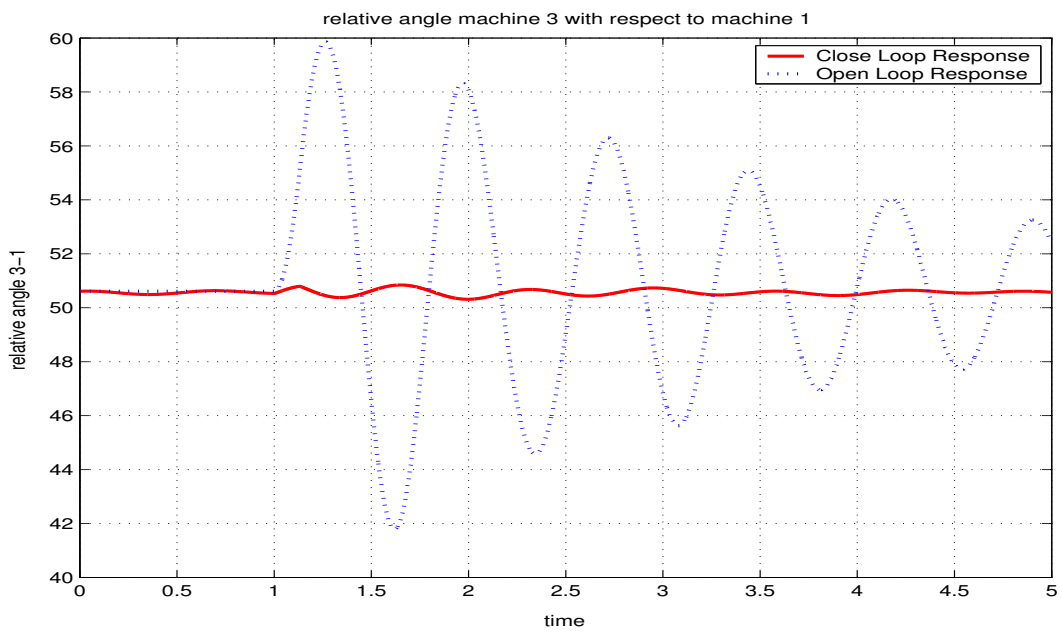

Figure 4.11: Case 1 Relative angle 3-1 


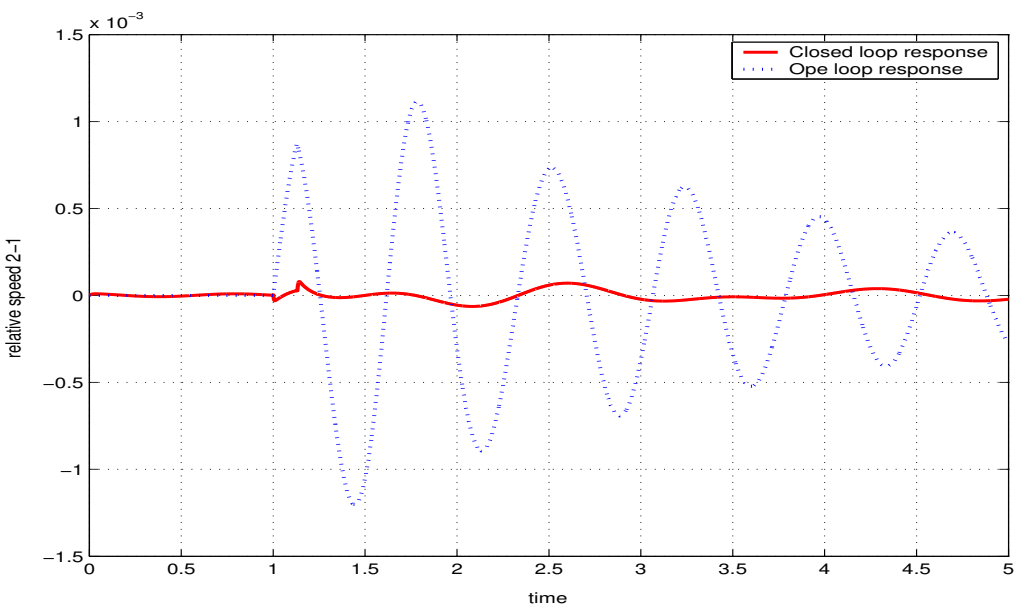

Figure 4.12: Case 2 Relative speed 2-1

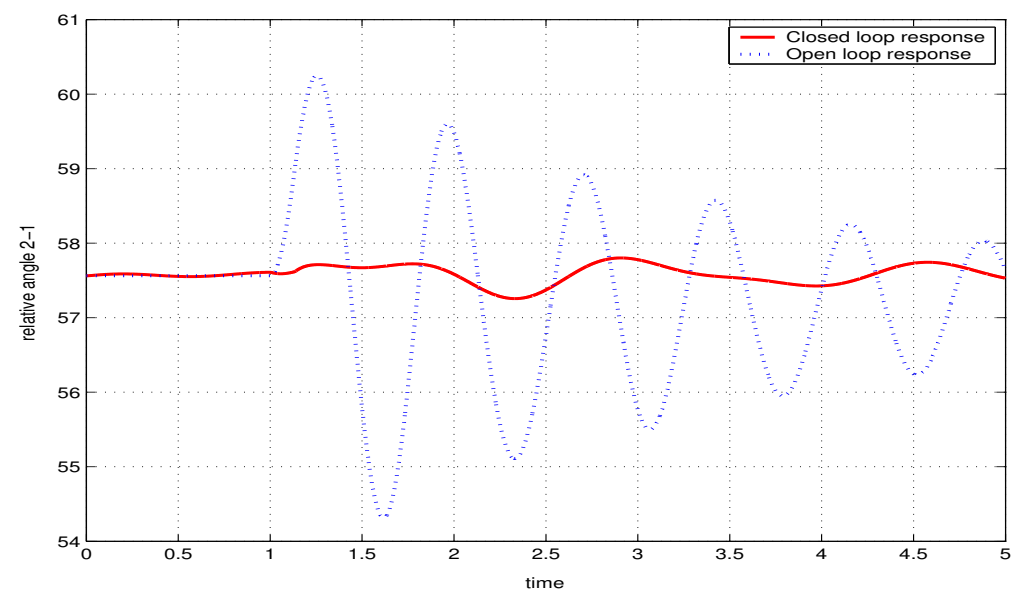

Figure 4.13: Case 2 Relative angle 2-1 


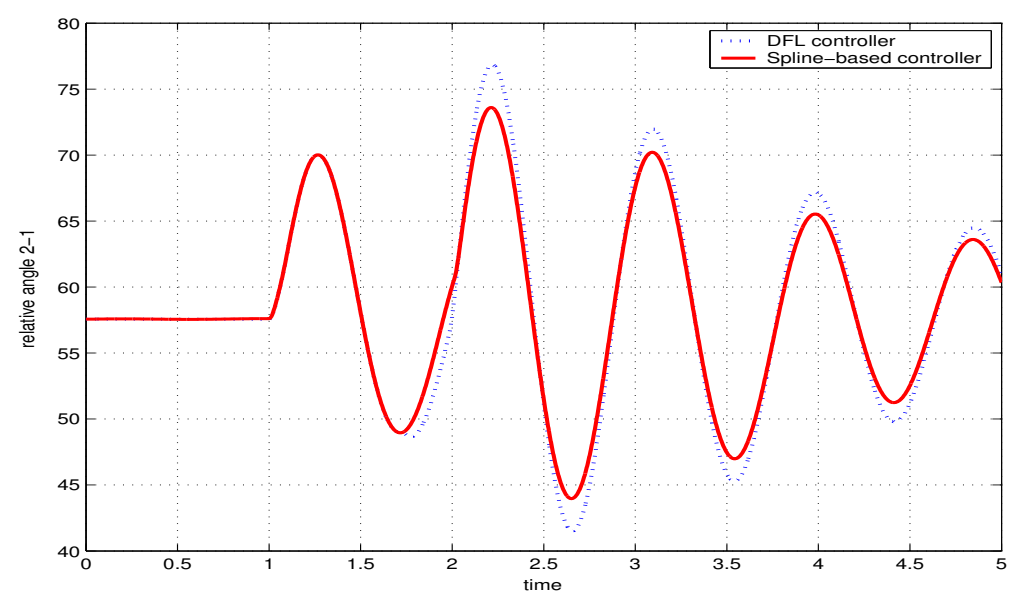

Figure 4.14: Case 3 Relative angle 2-1

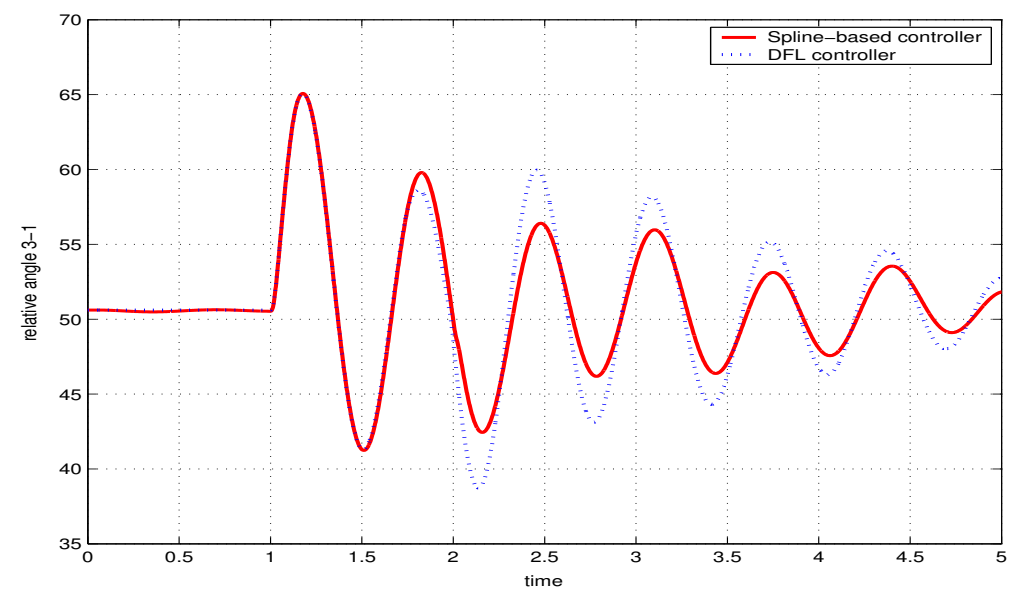

Figure 4.15: Case 3 Relative angle $3-1$

\subsubsection{Case 3 - Line to Ground Fault}

In this case the performance of DFL and spline based controller are compared with each other. Line to ground fault is applied on line $5-7$ for 0.15 seconds. Figure 4.14 and Figure 4.15 compare relative angle for closed loop responses. First, DFL controller applied. Then nonlinear controller for each generator with DFL method is approximated with piecewise polynomial with cubic spline technique. Approximated controller enhances stability of the system. Case 3 shows that spline based controller has better performance in comparisom to DFL method as can be seen by the oscillation of rotor angle.

Figure 4.16 compares spline based controller signal with DFL controller signal which applies 


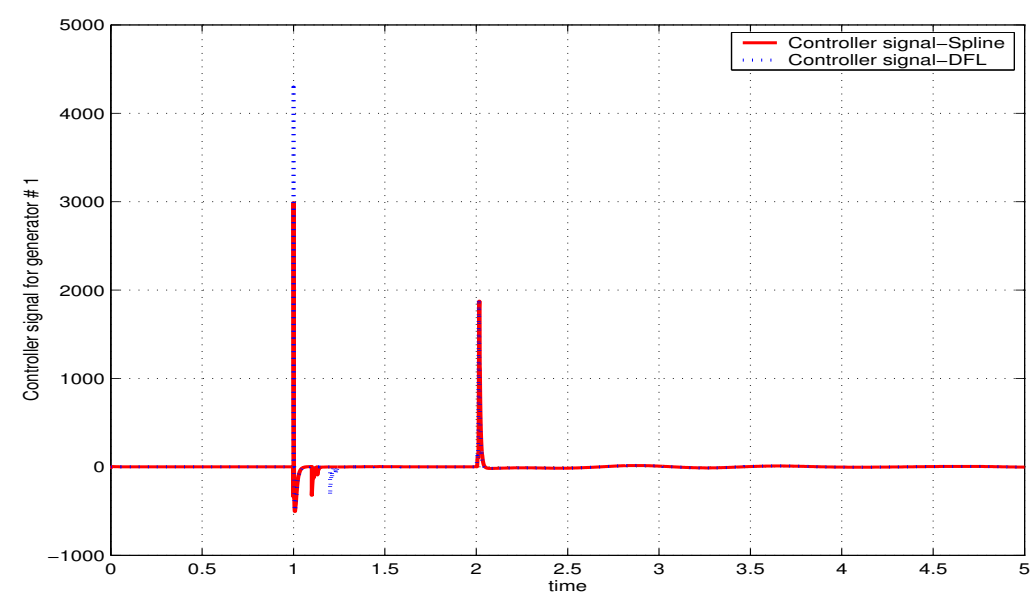

Figure 4.16: Case 3 Controller signals for generator 1

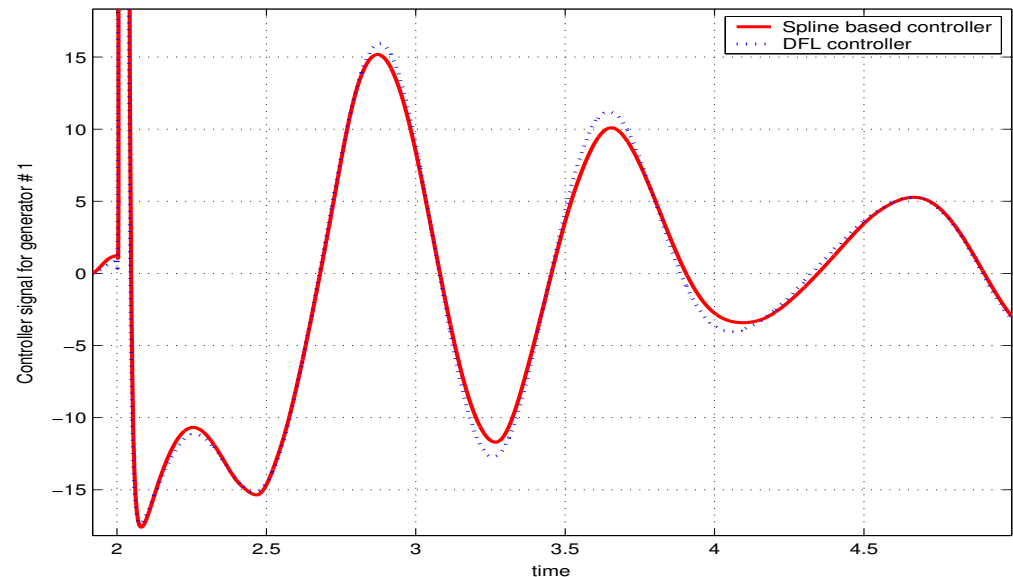

Figure 4.17: Case 3 Controller signals for generator 1 (detail)

to generator number 1. DFL controller signal has larger pick in compare to spline controller. For this case, rate limiter does not include for control signals. By zooming in Figure 4.16, difference between two controller signals can specified. Figure 4.17 and Figure 4.18 are focused for time interval $1.1-2.1$ and $2-5$ second.

Comparison between controllers for generator 2 and 3 is also shown in Figures 4.19 and Figure 4.20. It is assumed that the working region for synchronous generators is $0<\delta<180$ in order to guarantee that $I_{q} \neq 0$ in 4.35 . But this assumption is only valid for the steady state. The problem can be solved by including saturation nonlinearity on the control signal. Saturation block limits the gain $\frac{1}{I_{q}}$ in the controller. 


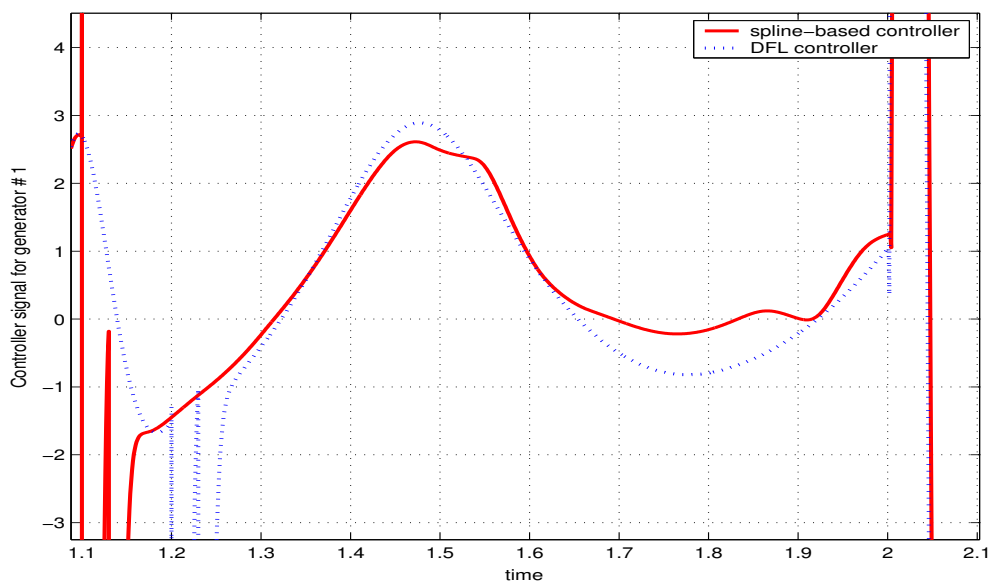

Figure 4.18: Case 3 Controller signals for generator 1 (detail)

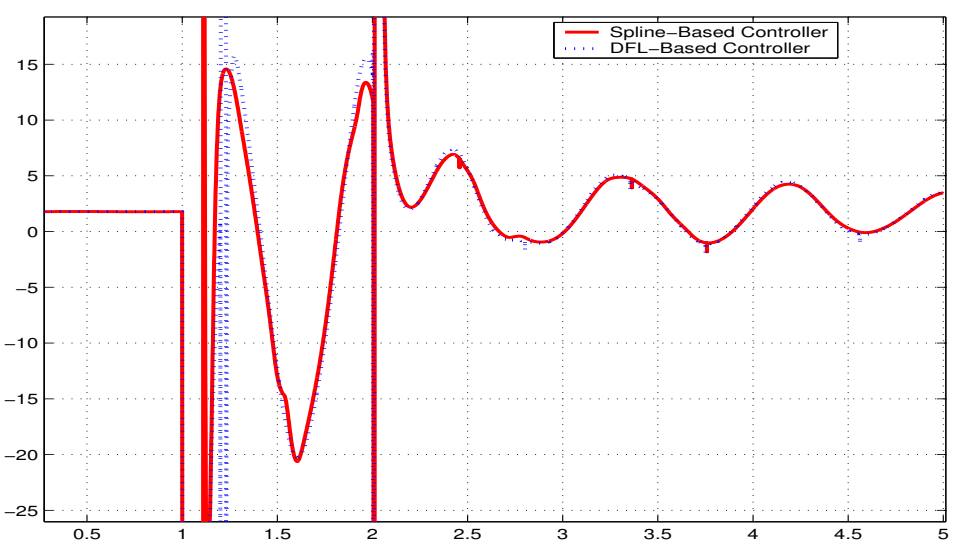

Figure 4.19: Case 3 Controller signals for generator 2 


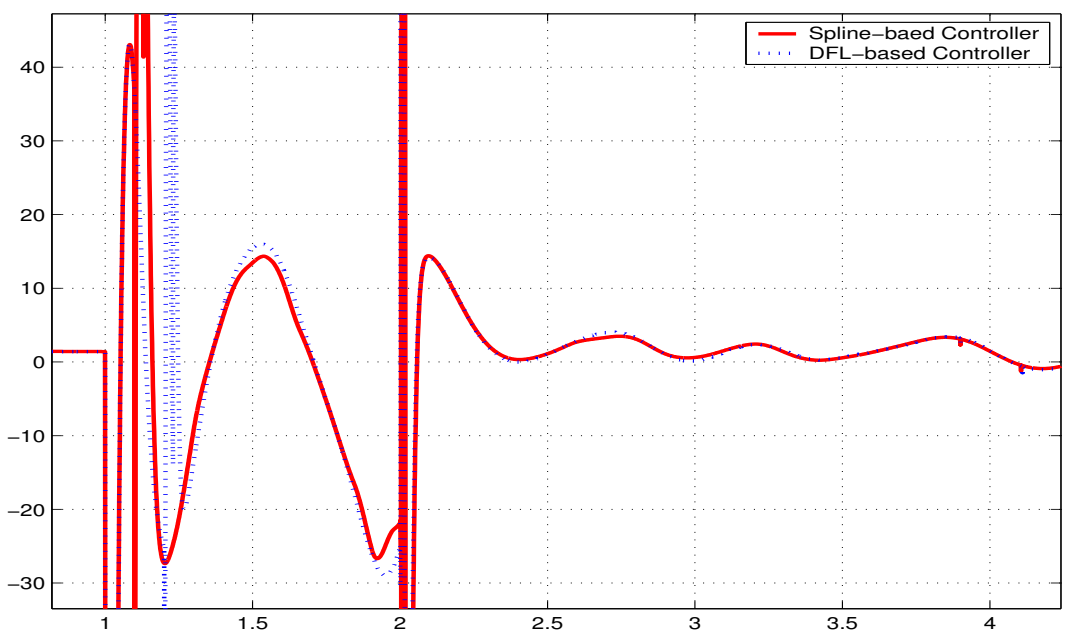

Figure 4.20: Case 3 Controller signals for generator 3

\subsection{Spline Controller}

Controller with DFL method is a nonlinear controller given by

$$
E_{f l d_{i}}=\text { Linear Compensator }+ \text { Nonlinear Compensator }
$$

Two approximation methods are applied to DFL excitation controller. First, the DFL excitation controller is approximated by cubic spline. For each independent variable of controller considered 20 number of sampled data. Assuming that the sampled data can cover the variation for each independent variable. This means that twenty sequences of data within a maximum and minimum variation are limited within a bound. Apply knot averages method to these set of data. Construct all the combination sets of these which construct the spline space. The linear compensator is computed for the discretized three dimension space. The same procedure is also applied to nonlinear compensator. The final objective of this sampling, knot averaging, and finally cubic spline interpolation, is to reconstruct the overall function in form piecewise polynomials which approximate DFL excitation controller.

Another method of computing splines to approximate functions is B-splines. This method is based on constructing the basis for the vector space of splines defined on interval $[a, b]$ and then solving a system of linear equations for the coefficients of the desired approximants in new basis. 
The basis function are known as B-splines. The "B" declares that these splines form a basis and they tend to have bell-shaped graphs. Linear system of equations can be solved very efficiently. B-splines can be defined in various ways, including recursive, convolution, and divided differences. Here, the recursive method is considered. Mathematical notation for an arbitrary function $f$ of order $k$ for knot sequence $t$ written as $f \in S_{k, t}$. This expresses that $f$ is a linear combination of B-splines of order $k$ with knot sequence $t$. In particular, B-form presents the spline as a weighted sum of B-splines with order $k$. It can be written as summation

$$
\sum_{j=1}^{n} B_{j, k} \alpha_{j}
$$

Here $B_{j, k}$ is the $j^{t h}$ B-spline of order $k$ that depends on the knot sequence $t_{1}<t_{2}<\ldots<t_{n+k}$. There are some definitions related to this mathematical formulation which are explained as follow $\alpha_{j}$ are $n$ real numbers which are called control points.

$k$ is the order of the polynomial segment of the B-splines curve.

Order $k$ means that the curve is made up of piecewise polynomial segments of degree $k-1$. $B_{j, k}$ are normalized B-splines functions. These functions are described by the order $k$ and by non decreasing sequence of real numbers which are called knot sequences $t_{i}: i=0 \ldots n+k$.

Basically $B_{j, k}$ is piecewise polynomial of degree $<k$ with breaks $t_{j}, \ldots, t_{j+k}$, is nonnegative and zero outside the interval $\left(t_{j} \ldots t_{j+k}\right)$. The summation is normalized in a way that for interval $\left[t_{k} \ldots t_{n+1}\right]$ the summation of splines will become 1 .

$$
\sum_{j=1}^{n} B_{j, k}=1
$$

Which is one important property of B-splines function. Recursive definition for B-splines is given next

For the case where $k=1$ the $j^{\text {th }} \mathrm{B}$-spline of degree 1 is defined by

$$
B_{j, 1}=\left\{\begin{array}{ccc}
1 & \text { if } & x \in\left[t_{j}, t_{j+1}\right) \\
0 & \text { otherwise } & x \notin\left[t_{j}, t_{j+1}\right)
\end{array}\right.
$$

And for the case where $k>1$ then the $j^{\text {th }}$ B-spline of degree $k$ is defined by

$$
B_{j, k}(x)=\frac{x-t_{j}}{t_{j+k}-t_{j}} B_{j, k-1}(x)+\frac{t_{j+1+k}-x}{t_{j+1+k}-t_{j+1}} B_{j+1, k-1}(x)
$$

Note that the order $k$ is independent of the number of control points. For B-splines there is flexibility of using many control points while restricting the degree of polynomial for each segment. 


\subsubsection{Case 4 - Three Phase Fault}

B-splines approximation is applied to the same system and it will be compared with cubic spline approximation. Three phase fault is applied on line $5-7$. The fault is cleared after 0.15 seconds. Figures 4.21 and 4.22 show the comparison between oscillation of rotor angles of machines 2,3 with respect to machine 1 . The transient response for rotor angle oscillation obtained with B-splines controller is improved compared to cubic spline controller.

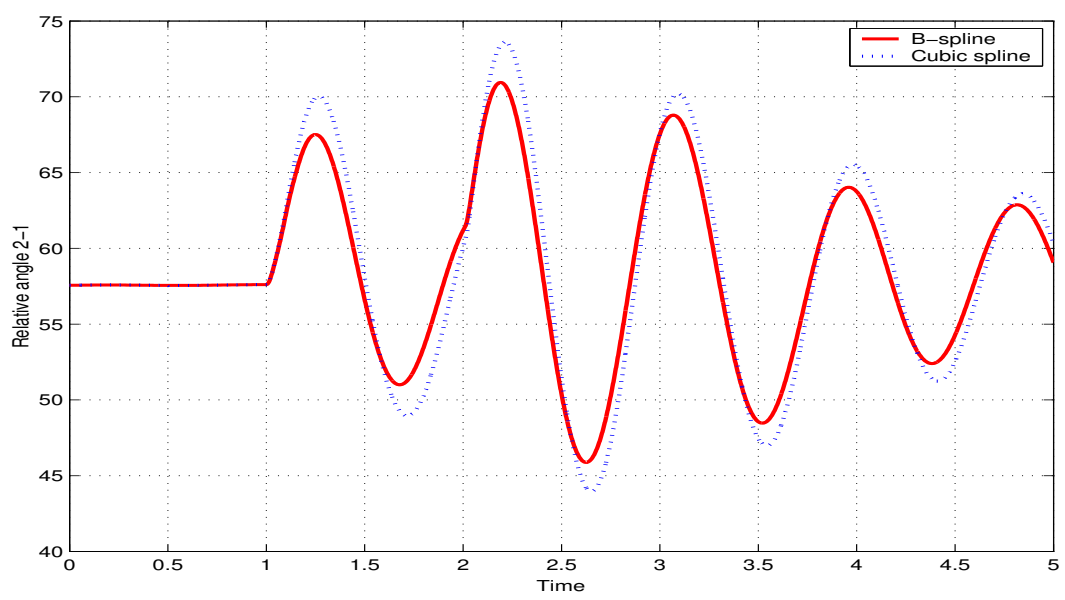

Figure 4.21: Case 4 Relative rotor angle 2-1

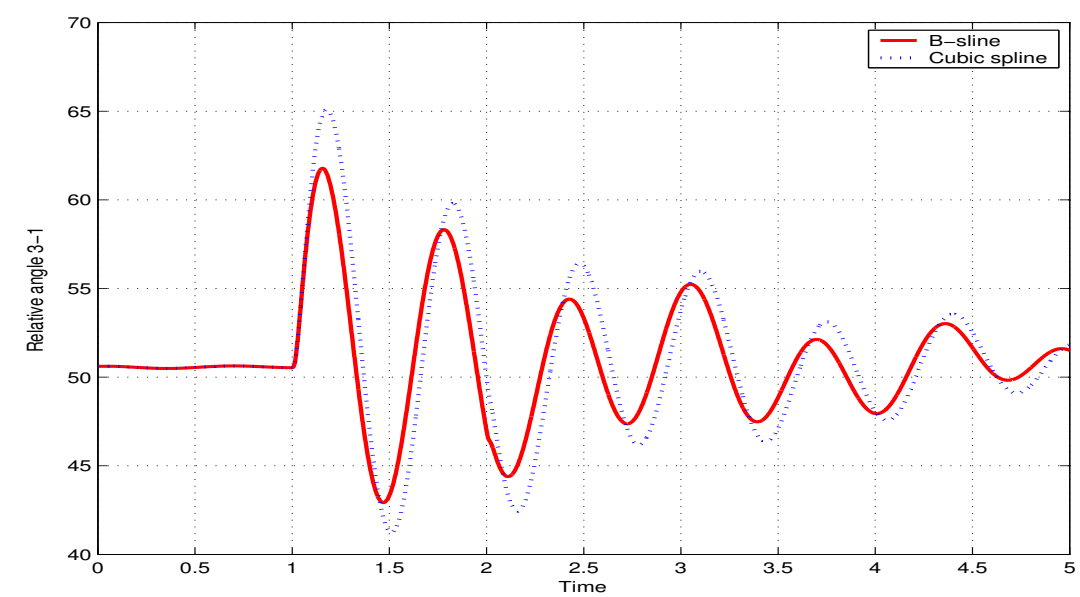

Figure 4.22: Case 4 Relative rotor angle 3-1 


\subsection{Internal Variable Identifier}

Direct feedback linearization is a nonlinear control design tool. From (4.35) controller for two-axis model generator needs $I_{q i}, I_{d i}, E_{q i}^{\prime}$, and $E_{d i}^{\prime}$ which are internal machine variables that cannot be measured directly [22]. A method is proposed to identify these internal variables. Its schematic block diagram is shown in Figure 4.23 . The input variables of the identifier, $\bar{u}_{i}$, are rate of change of active and reactive power, field voltage, and active power

$$
\bar{u}_{i}=\left(\begin{array}{cccc}
\dot{P}_{e i} & \dot{Q}_{e i} & E_{f l d_{i}} & P_{e i}
\end{array}\right)^{T}
$$

The state variables, $\hat{x}_{i}$, of the identifier are direct and quadrature currents, internal voltages, and speed

$$
\hat{x}_{i}=\left(\begin{array}{ccccc}
I_{d i} & I_{q i} & E_{q i}^{\prime} & E_{d i}^{\prime} & \omega_{i}
\end{array}\right)^{T}
$$

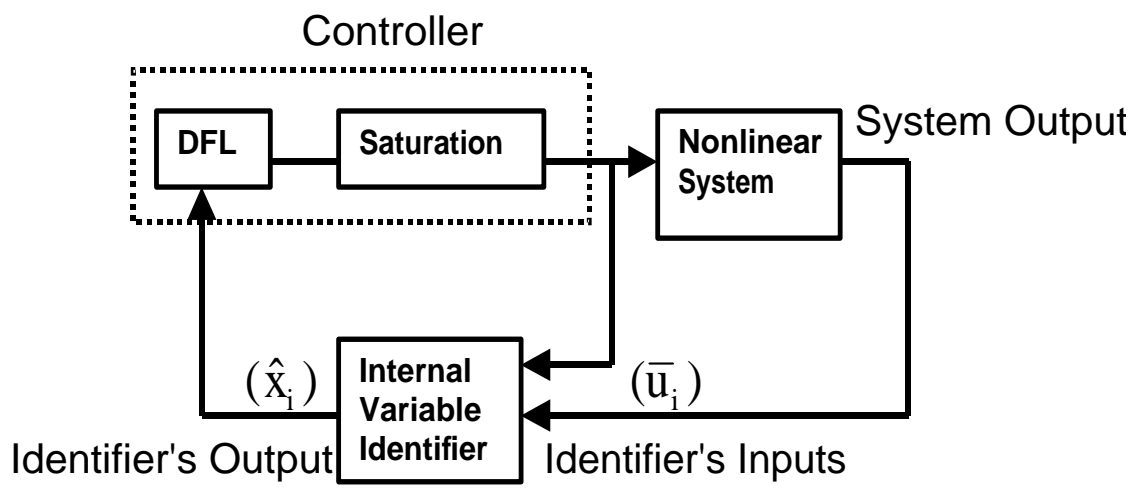

Figure 4.23: Internal variable identifier block diagram

The state equations for the identifier are of the form

$$
\dot{\hat{x}}_{i}=f_{i}\left(\hat{x}_{i}\right)+g_{i}\left(\hat{x}_{i}, \bar{u}_{i}\right)
$$

Which are derived as follows. Consider the equations for active and reactive power

$$
\begin{aligned}
P_{e i} & =E_{d i}^{\prime} I_{d i}+E_{q i}^{\prime} I_{q i} \\
Q_{e i} & =E_{q i}^{\prime} I_{d i}-E_{d i}^{\prime} I_{q i}
\end{aligned}
$$


Their rate of change is

$$
\begin{aligned}
& \dot{P}_{e i}=\dot{E}_{d i}^{\prime} I_{d i}+E_{d i}^{\prime} \dot{I}_{d i}+\dot{E}_{q i}^{\prime} I_{q i}+E_{q i}^{\prime} \dot{I}_{q i} \\
& \dot{Q_{e i}}=\dot{E}_{q i}^{\prime} I_{d i}+E_{q i}^{\prime} \dot{I}_{d i}-\dot{E}_{d i}^{\prime} I_{q i}-E_{d i}^{\prime} \dot{I}_{q i}
\end{aligned}
$$

Substitute equations $4.13,4.14$ into $4.55,4.56$ and rearrange to yield $\dot{I}_{d i}$ and $\dot{I}_{q i}$.

$$
\begin{aligned}
\dot{I}_{d i}= & {\left[\dot{P}_{e i} E_{d i}^{\prime}-\left(-E_{d i}^{\prime}+\left(X_{q i}-X_{q i}^{\prime}\right) I_{q i}\right) I_{d i} E_{d i}^{\prime} \frac{1}{T_{q i}}+\right.} \\
+\dot{Q}_{e i} E_{q i}^{\prime} & -I_{q i} E_{d i}^{\prime}\left(-E_{q i}^{\prime}-\left(X_{d i}-X_{d i}^{\prime}\right) I_{d i}+E_{f l d_{i}}\right) \frac{1}{T_{d i}}- \\
& -\left(-E_{q i}^{\prime}-\left(X_{d i}-X_{d i}^{\prime}\right) I_{d i}+E_{f l d_{i}}\right) I_{d i} E_{q i}^{\prime} \frac{1}{T_{q i}}+ \\
& \left.+I_{q i} E_{q i}^{\prime}\left(-E_{d i}^{\prime}+\left(X_{q i}-X_{q i}^{\prime}\right) I_{q i}\right) \frac{1}{T_{q i}}\right] \frac{1}{E_{q i}^{\prime 2}+E_{d i}^{\prime 2}} \\
\dot{I}_{q i}= & {\left[\dot{P}_{e i} E_{q i}^{\prime}-\left(-E_{d i}^{\prime}+\left(X_{q i}-X_{q i}^{\prime}\right) I_{q i}\right) I_{d i} E_{q i}^{\prime} \frac{1}{T_{q i}}-\right.} \\
\dot{Q}_{e i} E_{d i}^{\prime}- & I_{q i} E_{q i}^{\prime}\left(-E_{q i}^{\prime}-\left(X_{d i}-X_{d i}^{\prime}\right) I_{d i}+E_{f l d_{i}}\right) \frac{1}{T_{d i}}+ \\
& +\left(-E_{q i}^{\prime}-\left(X_{d i}-X_{d i}^{\prime}\right) I_{d i}+E_{f l d_{i}}\right) I_{d i} E_{d i}^{\prime} \frac{1}{T_{d i}}- \\
& \left.-I_{q i} E_{d i}^{\prime}\left(-E_{d i}^{\prime}+\left(X_{q i}-X_{q i}^{\prime}\right) I_{q i}\right) \frac{1}{T_{q i}}\right] \frac{1}{E_{q i}^{\prime 2}+E_{d i}^{\prime 2}}
\end{aligned}
$$

In summary, the system of nonlinear differential equations given by 4.12 , 4.13, 4.14, (4.57), and 4.58) is the state space representation of the identifier. This method is tested for three machines nine bus test system. Evaluation for performance of the identifiers is assessed. Figures 4.24, 4.25, and 4.26 compare exact and identified values for $I_{d i}, I_{q i}$, and $\omega_{i}$. The major difference for direct and quadrature currents happens at the time when fault occurs. 

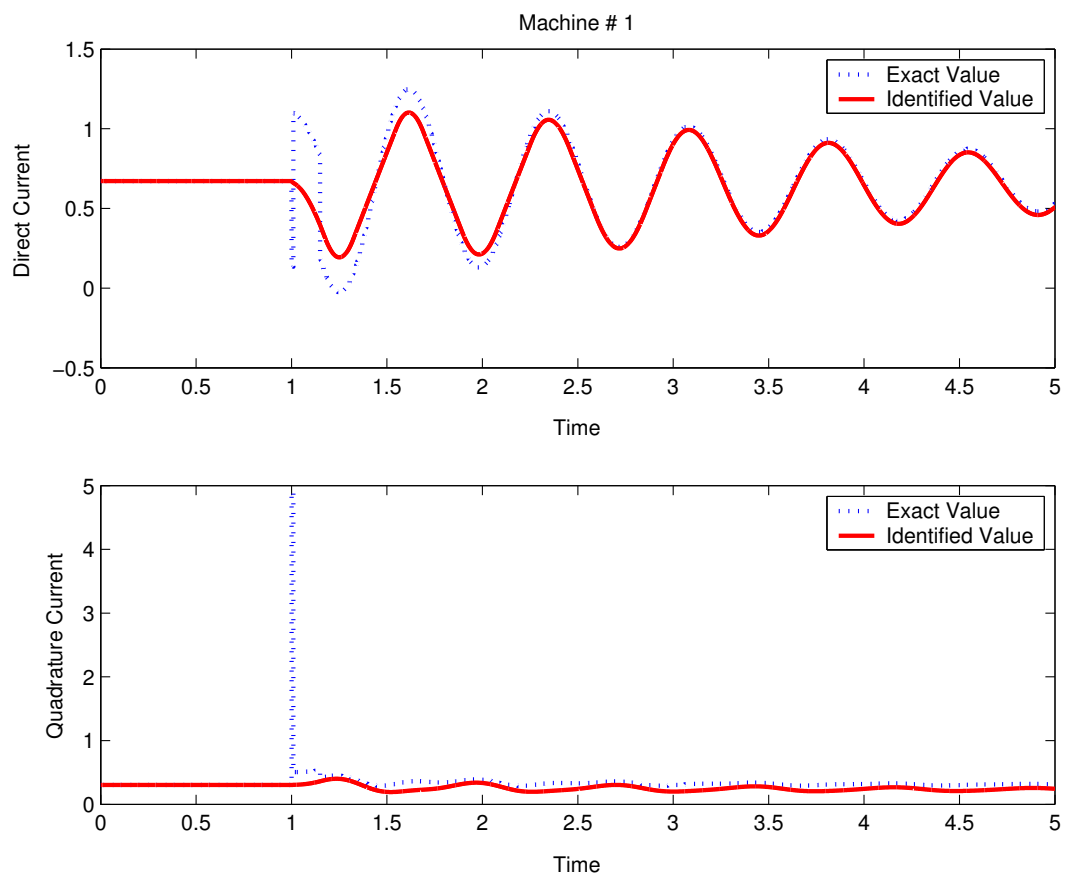

Figure 4.24: Direct and quadrature current for machine 1
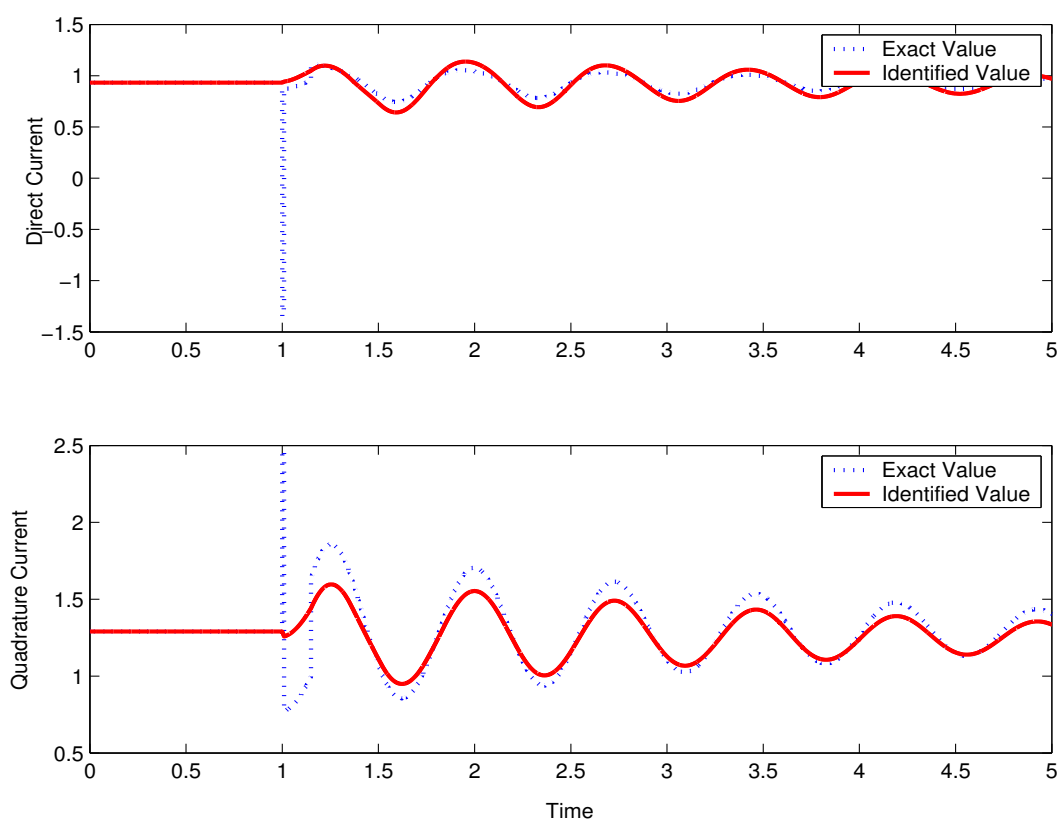

Figure 4.25: Direct and quadrature current for machine 3 

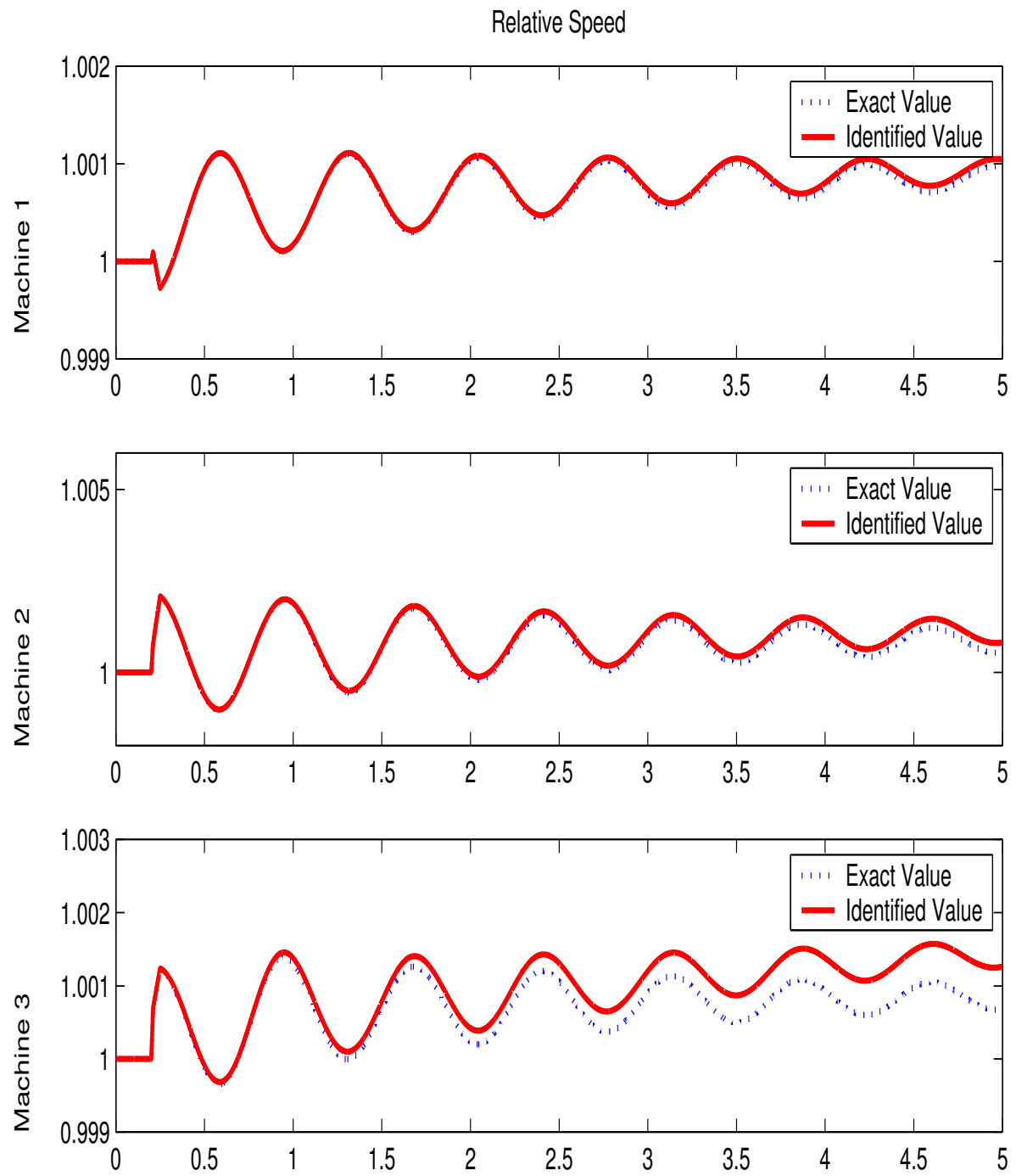

Figure 4.26: Speed of the machines 


\subsection{Controller Tuning - Genetic Algorithm}

Genetic algorithm can be used to solve a vast variety of optimization problems. In brief, the method is a probabilistic optimization technique which is based on the natural evolution process. In this technique, fitness function has the same role as objective function for the traditional optimization problem. There are some basic definitions that are commonly used in genetic optimization and are given briefly in the following

Individual - Set of variables that need to be optimized.

Generation - Summation for the set of individuals.

Selection - Collect the best individuals in current population and use it for generating next population.

Crossover - Make large set of individual to exchange a subset of the genetic information with each other.

Mutation - Causes individual genetics changes according to some probabilistic rules.

Objective function - It is used to provide a measure of how individuals have performed in problem domain.

Fitness function - It corresponds to the number of offspring that an individual can expect to produce in next generation.

In genetic optimization method, individuals with better fitness values are accepted and those with low fitness values are rejected. So, the final solution will become individual with best fitness value. This final solution may not be unique, and mainly depends on problem formulation. In control theory, genetic algorithm can also be used to obtain the control gains such that the closed loop eigenvalues of linear system lie on the left hand side of $j \omega$ axis of s-complex plane. Basically the algorithms are global search techniques and more likely to converge to global optima than conventional optimization techniques. It has already been used in complicated multidimensional problems. Conventional optimization techniques, such as output feedback, are based on deterministic method which may find local solution instead of global while genetic algorithms based global solutions [1, 11, 8].

In genetic algorithm, fitness function is defined in a way that will minimize the real part of closed loop eigenvalues for each subsystem as defined by 


$$
\begin{array}{r}
A_{c l i}=A_{i}+B_{i} K_{i} \\
\text { fitness function }=\max \left[\operatorname{Re}\left\{\text { eig }\left(A_{\text {cli }}\right)\right\}\right] \\
\text { subject to } K_{i} \in[-\alpha, \alpha]
\end{array}
$$

Where $\alpha$ is the bound for controller gains. The controller gains are found by minimizing the fitness function. The fitness function should give domain-specific information about each individual. So it is proper to define it in form of a mathematical formulation, either a maximization or minimization of some parameters and constrains.

Genetic algorithms are very efficient method in finding the optimization solution to nonlinear and non-differentiable problems. The size of problems that can be solved using genetic algorithms is a lot greater than maximum size of problems that can be solved using other techniques. The algorithm is capable of handling harder and larger size problem, and it is flexible in terms of the size of populations being used, the cross over and mutation probabilities and their methods. For more complicated problems, the algorithm can be easily modified.

\subsubsection{Case Study}

The three machine nine bus test system is used to illustrate the proposed approach. First a feedback linearized system is obtained for each machine then genetic search optimization method is applied in order to tune the controller gains. Limits on the gains are imposed within a limited bound $K_{i}=[-150,150]$. The fitness function is defined in a way that will minimize the real part of closed loop eigenvalues for each subsystem [1, 11].

The gains are obtained as follows

$$
\begin{aligned}
& K_{1}=\left(\begin{array}{lll}
-98.3322 & -134.3784 & -0.4811
\end{array}\right) \\
& K_{2}=\left(\begin{array}{lll}
-138.0316 & -131.9364 & -2.7827
\end{array}\right) \\
& K_{3}=\left(\begin{array}{lll}
-143.9325 & -112.3946 & -4.6062
\end{array}\right)
\end{aligned}
$$


Comparison is done between open-loop and close-loop eigenvalues for each subsystem. Open loop means the subsystem itself without any controller as eig $\left(A_{i}\right)$ and closed loop means that once the gains are obtained by genetic algorithm the closed loop eigenvalues are obtained as eig $\left(A_{i}+B_{i} K_{i}\right)$.

\section{Subsystem - 1}

$$
\begin{aligned}
\text { eig } & =\left(\begin{array}{lll}
0 & -0.0212 & -3.2258
\end{array}\right) \\
\text { eig } &
\end{aligned}
$$

\section{Subsystem - 2}

$$
\begin{aligned}
\text { eig } g_{\text {open }} & =\left(\begin{array}{lll}
0 & -0.0781 & -1.8692
\end{array}\right) \\
\text { eig } g_{\text {close }} & =\left(\begin{array}{lll}
-2.3828 & -2.3830+1.6668 i & -2.3830-1.6668 i
\end{array}\right)
\end{aligned}
$$

\section{Subsystem - 3}

$$
\begin{aligned}
\text { eig } g_{\text {open }} & =\left(\begin{array}{lll}
0 & -0.1661 & -1.6667
\end{array}\right) \\
\text { eig close } & =\left(\begin{array}{lll}
-3.1702 & -3.1699+1.5877 i & -3.1699-1.5877 i
\end{array}\right)
\end{aligned}
$$

Using the gains in (4.35), the controller can be obtained as

$$
\begin{gathered}
E_{f l i d_{1}}=\frac{28.9 V_{f 1}(t)}{I_{q 1}(t)}-28.9032 E_{q 1}^{\prime}+\frac{20.7062}{I_{q 1}}-8.96 E_{d 1}^{\prime} \frac{\dot{I}_{d 1}}{I_{q 1}}-8.96 E_{q 1}^{\prime} \frac{\dot{I}_{q 1}}{I_{q 1}}+0.0852 I_{d 1} \\
E_{f l i d_{2}}=\frac{11.21 V_{f 2}(t)}{I_{q 2}(t)}-7.49 I_{d 2}-11.214 E_{q 2}^{\prime}+\frac{18.2803}{I_{q 2}}-6 E_{d 2}^{\prime} \frac{\dot{I}_{d 2}}{I_{q 2}}-6 E_{q 2}^{\prime} \frac{\dot{I}_{q 2}}{I_{q 2}}+0.6562 I_{d 2}(4.64) \\
\left.E_{f l i d_{3}}=\frac{9.81 V_{f 3}(t)}{I_{q 3}(t)}-9.8932 I_{d 3}-9.816 E_{q 3}^{\prime}+\frac{8.3441}{I_{q 3}}-5.89 E_{d 3}^{\prime} \frac{\dot{I}_{d 3}}{I_{q 3}}-5.89 E_{q 3}^{\prime} \frac{\dot{I}_{q 3}}{I_{q 3}}+1.1312 I_{d 34} .65\right)
\end{gathered}
$$


The three phase symmetrical fault is applied on line $5-7$ at time $t=1$ second. It is removed after 0.15 seconds. The nonlinear controllers designed in the previous section are applied using outputs of the identifiers. The control signals for generators 1, 2, and 3 are shown in Figures 4.27, 4.28, 4.29. Field voltage signals are limited within specified bound. Excitation field voltage with DFL controller reaches the pre-fault steady state values after a short period of time, once the fault is cleared.

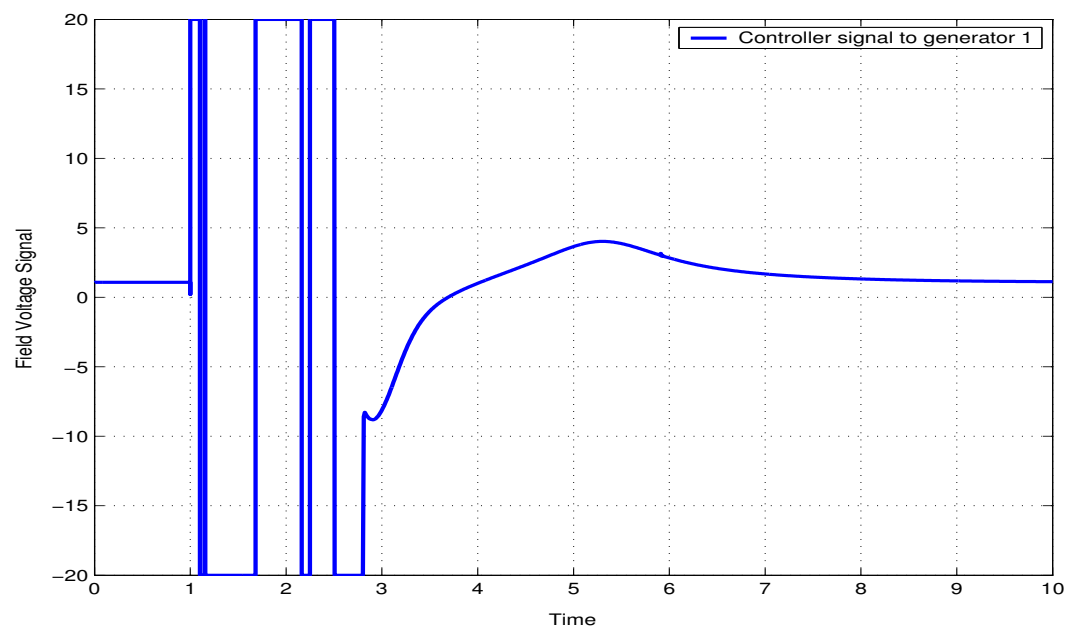

Figure 4.27: Control signal

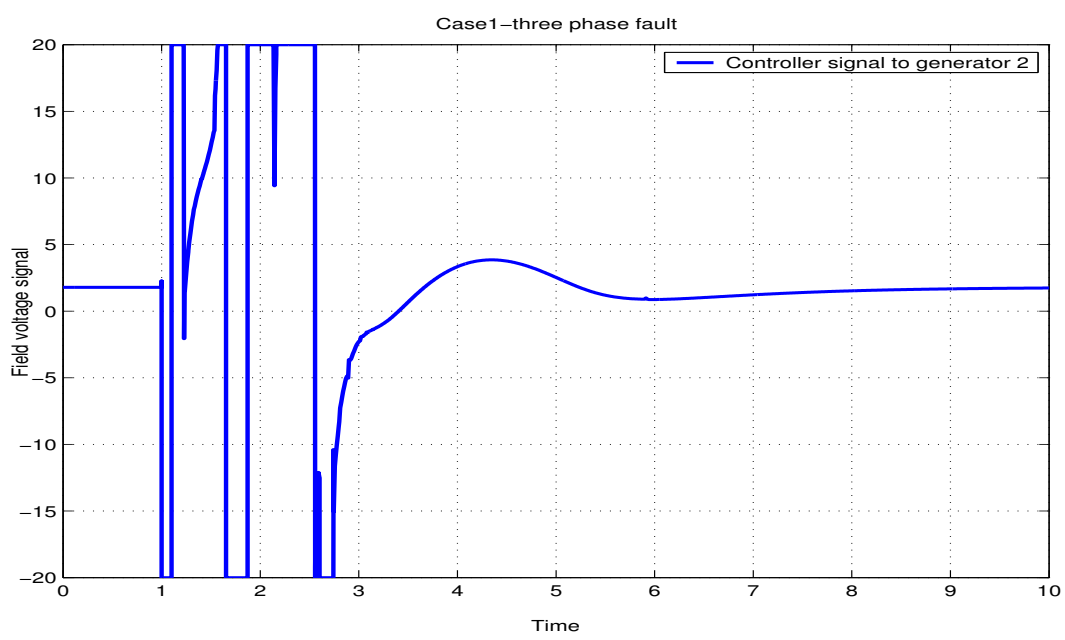

Figure 4.28: Control signal

Figures 4.30 and 4.31 show machine speed and rotor angle when the designed controllers are applied. Based on the first swing stability criteria, the generators will not loose their synchronism 


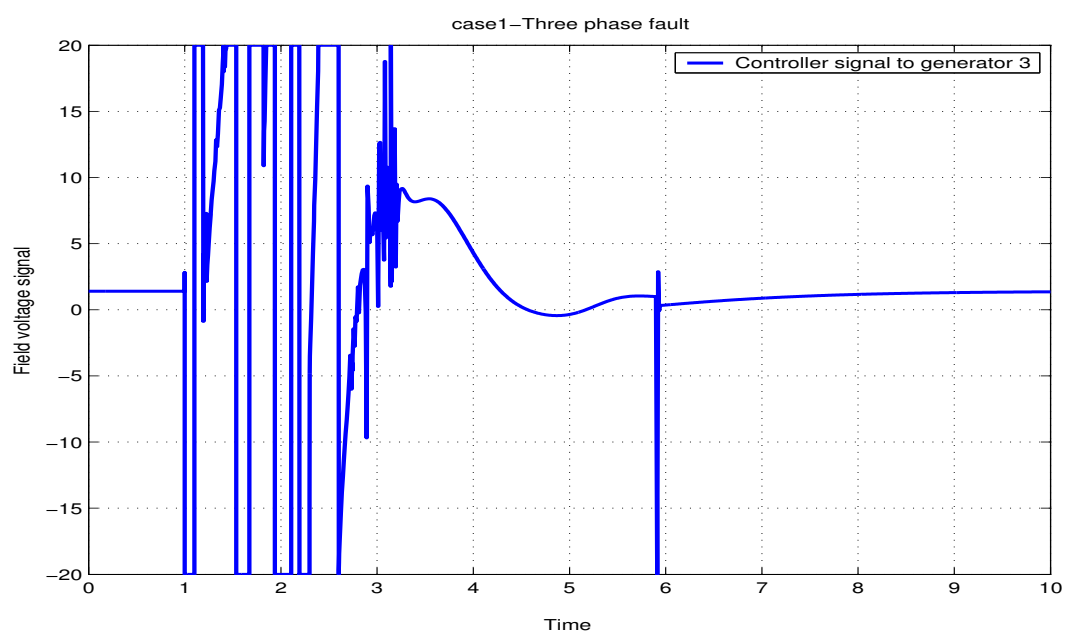

Figure 4.29: Control signal

for the first swing of the rotor angle as shown in Figures 4.31

The simulation shows that by using DFL excitation controller for two axis model, stability enhancement of the system is preserved in a case where large sudden fault occurs in the system. This case study shows control design for a nonlinear electric power system through the excitation loop of synchronous generator. Direct Feedback Linearization technique is presented and applied using a two-axis generator model.

DFL controller requires unmeasurable internal variables. Internal variable identifier block is presented in order to construct the unmeasurable variables for two axis model. The proposed method has been illustrated using a three-machine nine-bus test system. 


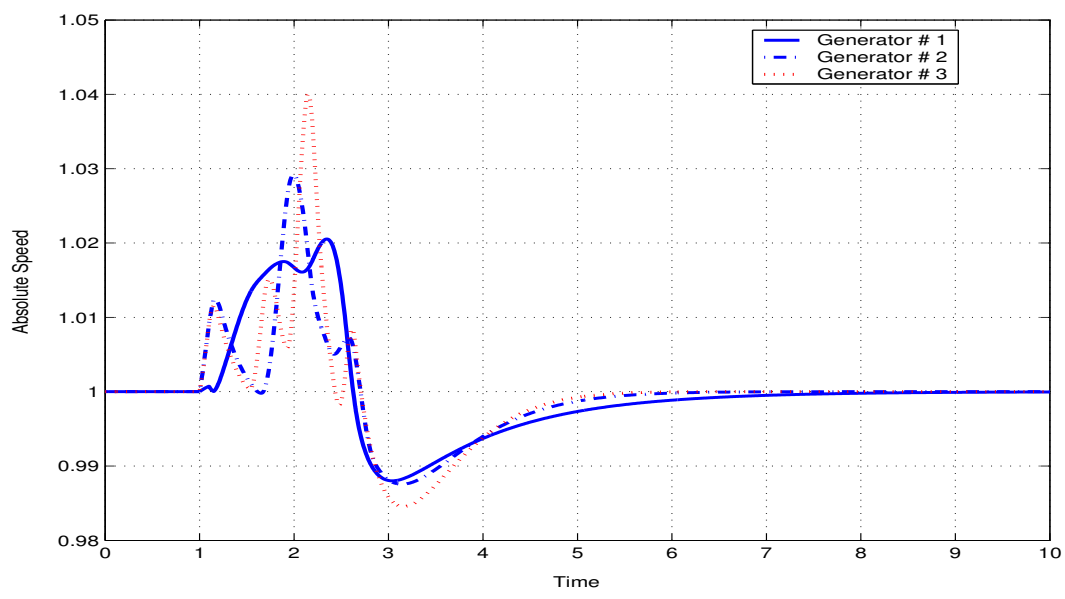

Figure 4.30: Speed of the machines

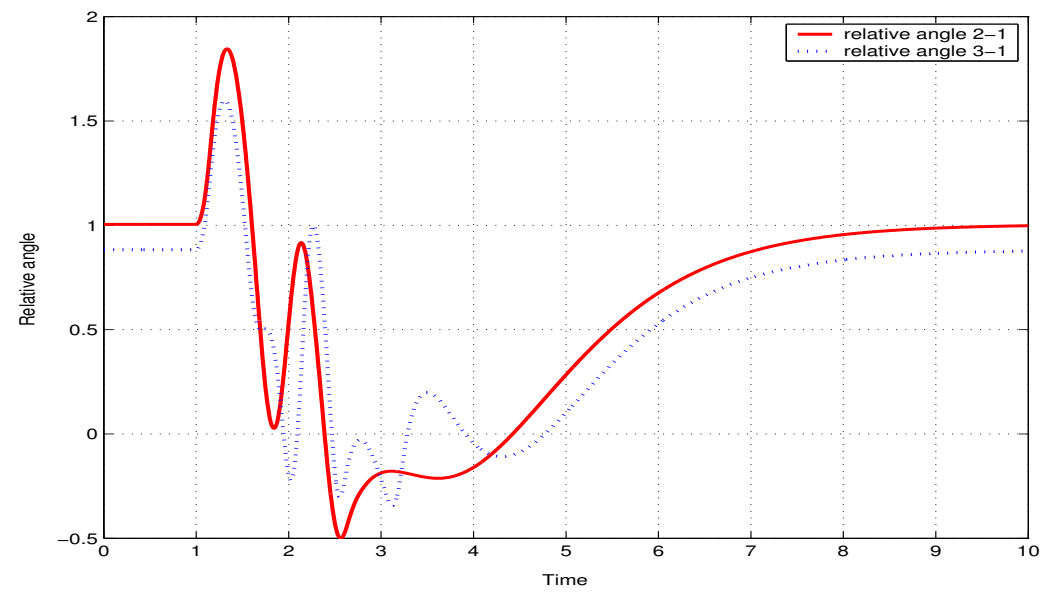

Figure 4.31: Relative rotor angle 


\subsection{Comparison between DFL Controller for One-Axis and Two- Axis Model}

For multi-machine power system, transient stability simulation is the most important tool for planning, design, and operation. Models of generator are considered as important factor with influence in simulation result. In this section, two types of generator models are covered with their own complexity of DFL controllers. Synchronous generator is the most important kind of the equipments in the electric power system. Performance of DFL controller is compared for two-axis and one-axis generator.

DFL controller has been applied to one-axis model previously by several researchers [16, 31, 30, 33]. One axis model generators have mathematical models slightly different from two axis. From the one axis model, it can be found that synchronous generator is also nonlinear through the excitation loop. Applying DFL technique the controller for excitation loop is given as

$$
E_{f l d i}=\frac{1}{I_{q i}}\left[W_{f i}(t)-E_{q i}^{\prime} \dot{I}_{q i} T_{d o i}^{\prime}+P_{m}\right]+I_{d i}\left(X_{d}-X_{d}^{\prime}\right)
$$

Similar to two-axis model, nonlinear generator model can also be linearized and decoupled as subsystem. Each subsystem has state space equation as follow

$$
\dot{x}_{i}=\bar{A}_{i} x_{i}(t)+\bar{B}_{i} W_{f i}(t)
$$

With

$$
\bar{A}_{i}=\left(\begin{array}{ccc}
0 & 1 & 0 \\
0 & -\frac{D_{i}}{2 H_{i}} & -\frac{\omega_{i 0}}{2 H_{i}} \\
0 & 0 & -\frac{1}{T_{d o i}^{\prime}}
\end{array}\right) \bar{B}_{i}=\left(\begin{array}{c}
0 \\
0 \\
\frac{1}{T_{d o i}^{\prime}}
\end{array}\right)
$$

Controller $W_{f i}(t)$ is

$$
W_{f i}=-\bar{K}_{i} x_{i}=-\bar{K}_{\delta_{i}} \Delta \delta_{i}-\bar{K}_{\omega_{i}} \Delta \omega_{i}-\bar{K}_{P e i} \Delta P_{e i}
$$

where $\bar{K}_{\delta i}, \bar{K}_{\omega i}$, and $\bar{K}_{P e i}$ are constant gains.

Classical three machine nine bus test system is considered here for comparison. Same controller 
design method, genetic algorithm, is applied for tuning static controller gains of $V_{f i}$ and $W_{f i}$ in DFL excitation controller. The fitness function is taken similar, which is to minimize the real part of closed loop eigenvalues for each subsystem. Controller gains are kept in same range for both models. The objective is to compare the effectiveness of controllers in transient stability enhancement of multi machine power system for different fault scenarios. Controller gains, obtained by genetic algorithm, within the bound of $\left[\begin{array}{lll}-100 & 100]\end{array}\right]$ are

One-Axis Model

$$
\begin{aligned}
\bar{K}_{1} & =\left(\begin{array}{lll}
11.4515 & 95.0126 & -5.8675
\end{array}\right) \\
\bar{K}_{2} & =\left(\begin{array}{lll}
29.1955 & 99.9998 & -10.4482
\end{array}\right) \\
\bar{K}_{3} & =\left(\begin{array}{lll}
43.6102 & 100 & -16.1482
\end{array}\right)
\end{aligned}
$$

Two-Axis Model

$$
\begin{aligned}
& K_{1}=\left(\begin{array}{lll}
53.1255 & 100 & -0.3964
\end{array}\right) \\
& K_{2}=\left(\begin{array}{lll}
85.2390 & 100 & -2.4267
\end{array}\right) \\
& K_{3}=\left(\begin{array}{lll}
81.0893 & 75.5685 & -3.5896
\end{array}\right)
\end{aligned}
$$

\subsubsection{Case 1 - Line to Ground Fault}

Line to ground fault is applied on line $5-7$. The fault is removed after 0.25 seconds. Figure 4.32 compare relative rotor angles. From rotor angle stability criteria, it is clear that two-axis model has advantage of preserving the stability of the system with smaller deviation in rotor angle. The control signals for generators 1 is shown in Figure 4.33. Excitation field voltage with DFL controller for two-axis model reaches the pre-fault steady state values after almost 3 second, while for one-axis model it takes almost 6 second with high deviation around steady state values.

\subsubsection{Case 2 - Three Phase Fault}

Three phase fault is applied on line $5-7$ for 0.15 seconds. Figure 4.34 compare the relative rotor angles for both systems. DFL excitation control for two-axis model is more accurate in compare to one-axis in the way that it can grasp detailed characteristic relevant to control design. 


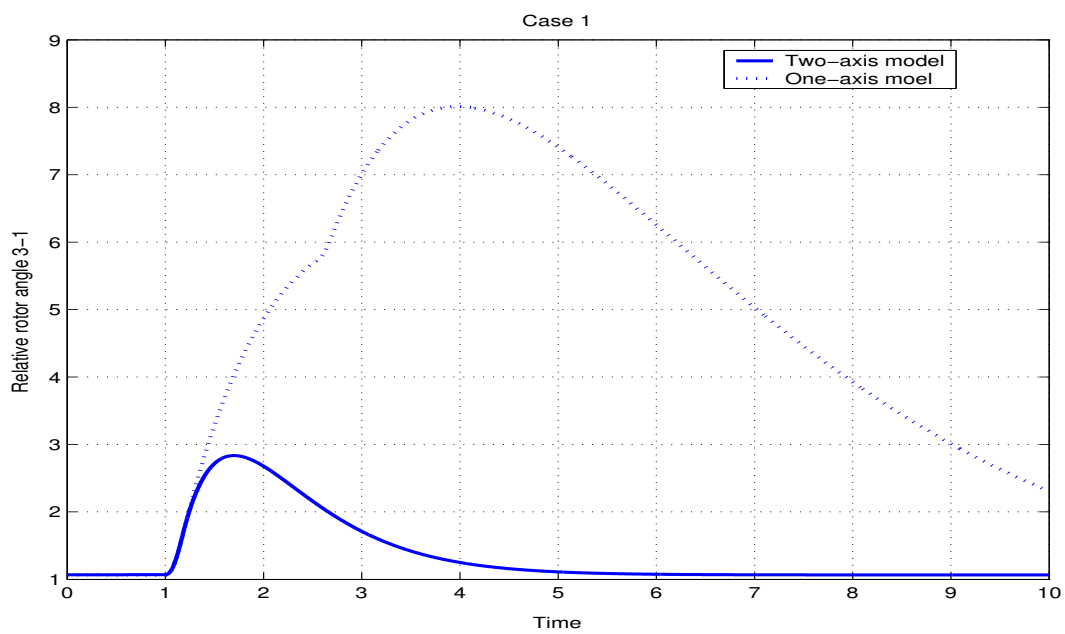

Figure 4.32: Comparison between relative rotor angle

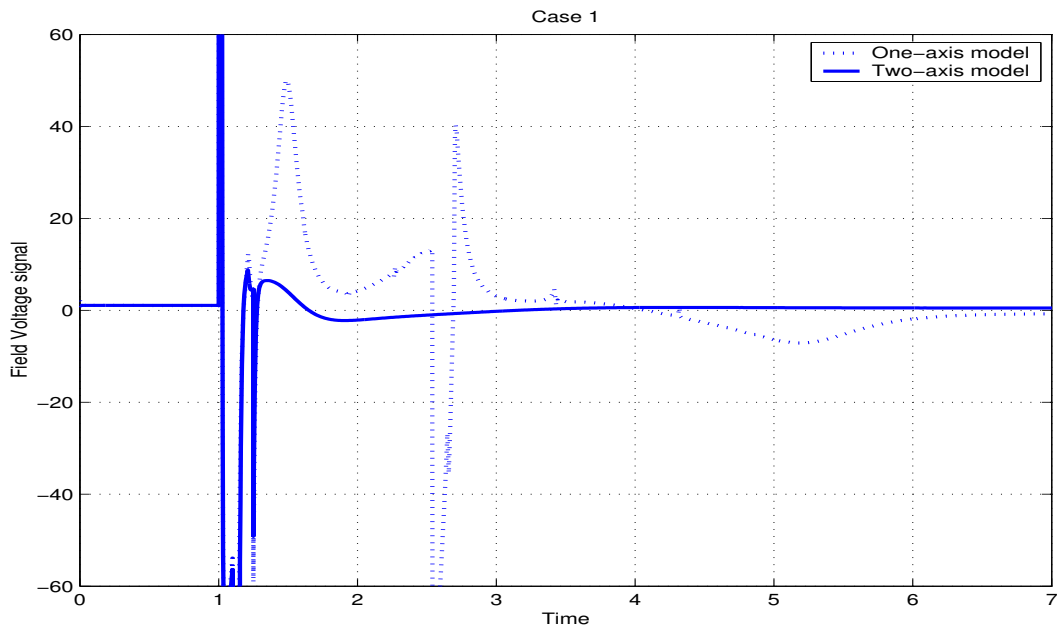

Figure 4.33: Comparison between control signal

Controller signal for generator 3 is also shown in Figure 4.35 which compares the response of DFL controller for two-axis and one-axis model.

\subsubsection{Comments}

There are several comments related to the previous case study specifically with tuning the controller gain with genetic algorithms and the effect of nonlinear saturation block on controller signals in place. These issues are addressed in the following

1. Genetic algorithm is used for tuning the controller gains under the same type of condi- 


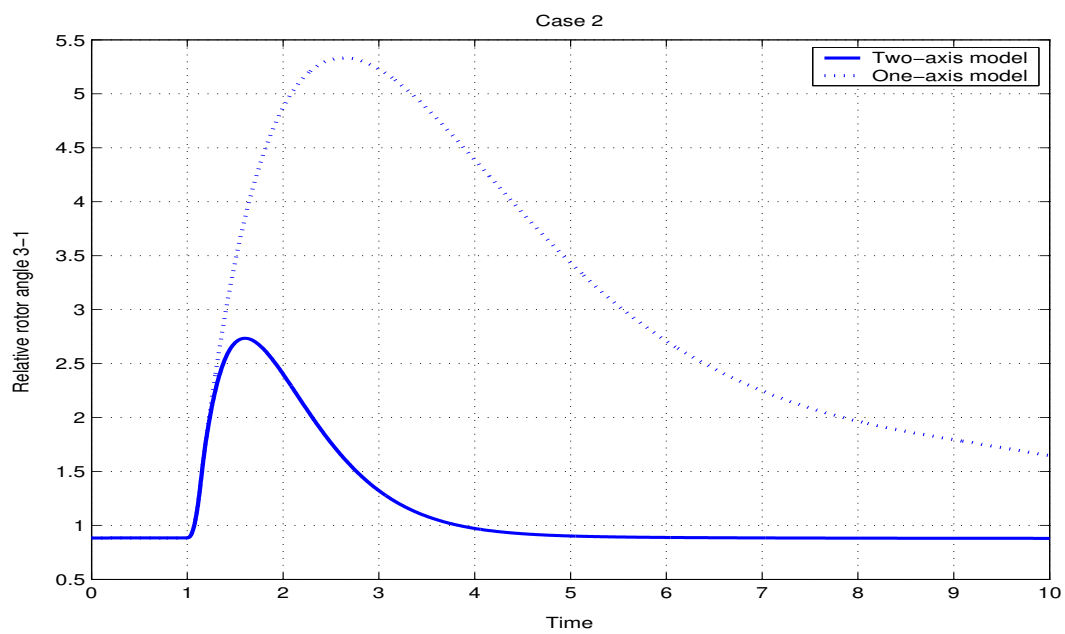

Figure 4.34: Comparison between relative rotor angle

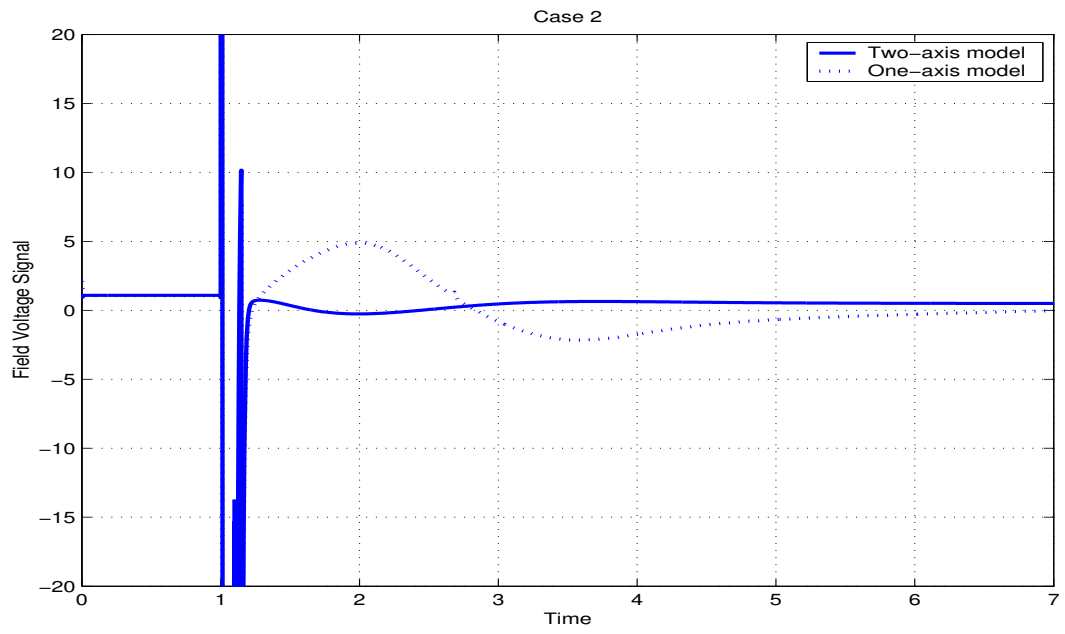

Figure 4.35: Comparison between control signals

tions. Fitness function is defined similarly for both decoupled system. Also the controller gains are bound within the same range.

2. For two-axis model, the complexity of DFL controller is increased when compared to oneaxis model. This is due to the more detailed dynamic modelling of the system.

3. Controller signals for two-axis model has smaller oscillation where compared to one-axis model, and tends to be more stable in compare to one-axis model. 
4. Nonlinear elements such as saturation block has direct influence on dynamic simulation of the system and also on the controller signals. For example in the three machine nine bus test system, consider a loss of line $5-7$ for 0.15 seconds. Controller gain is tuned by genetic algorithm. The gain is tuned within the bound $\left[\begin{array}{ll}-70 & 70\end{array}\right]$.

$$
\begin{aligned}
& \bar{K}_{1}=\left(\begin{array}{lll}
31.4076 & 66.5679 & -0.1043
\end{array}\right) \\
& \bar{K}_{2}=\left(\begin{array}{lll}
53.1455 & 69.9992 & -1.7910
\end{array}\right) \\
& \bar{K}_{3}=\left(\begin{array}{lll}
-38.489 & -47.2931 & -2.7501
\end{array}\right)
\end{aligned}
$$

Two different scenarios are compared for controller signals with and without saturation block. in Figures 4.36, 4.37, and 4.38 dotted line shows the controller signal with effect of saturation block. Solid line shows the controller signal without any saturation limit. The difference happens especially during the transient period.

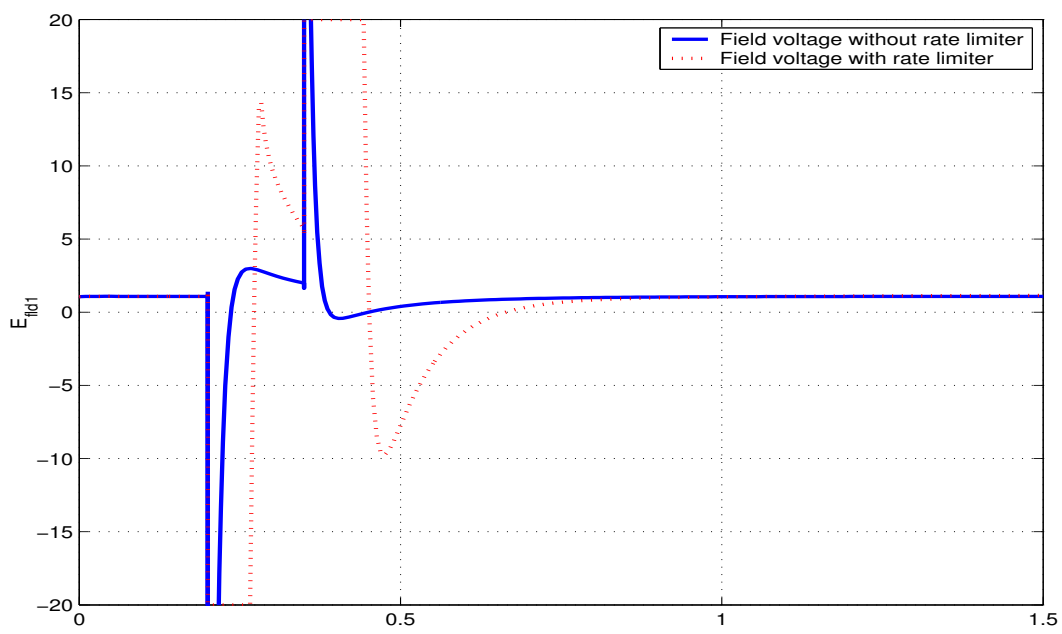

Figure 4.36: Effect of saturation block on first controller signal 


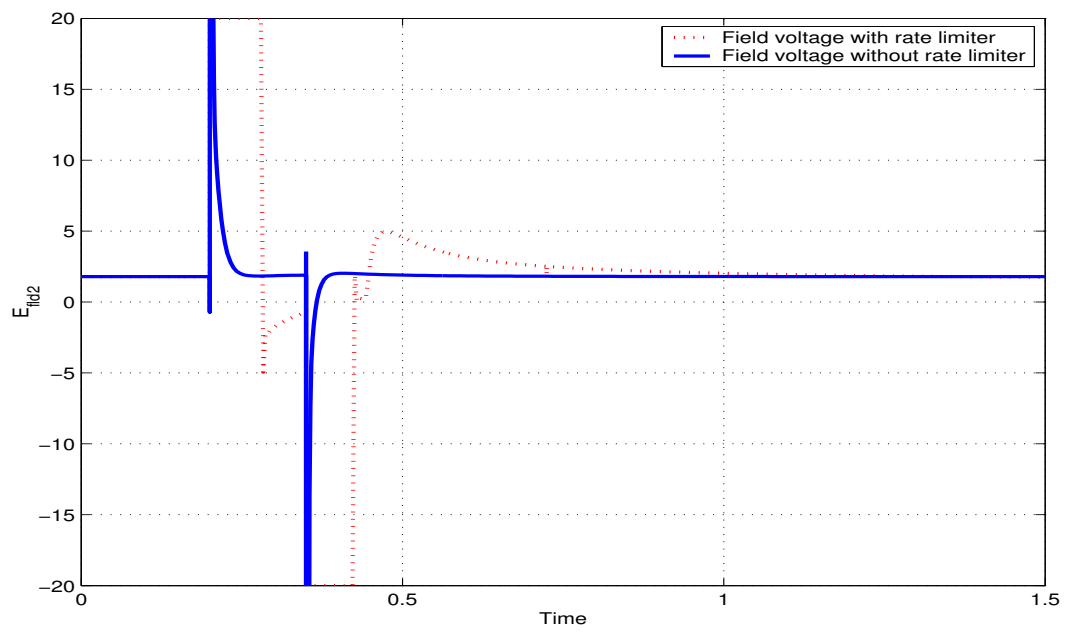

Figure 4.37: Effect of saturation block on second controller signal

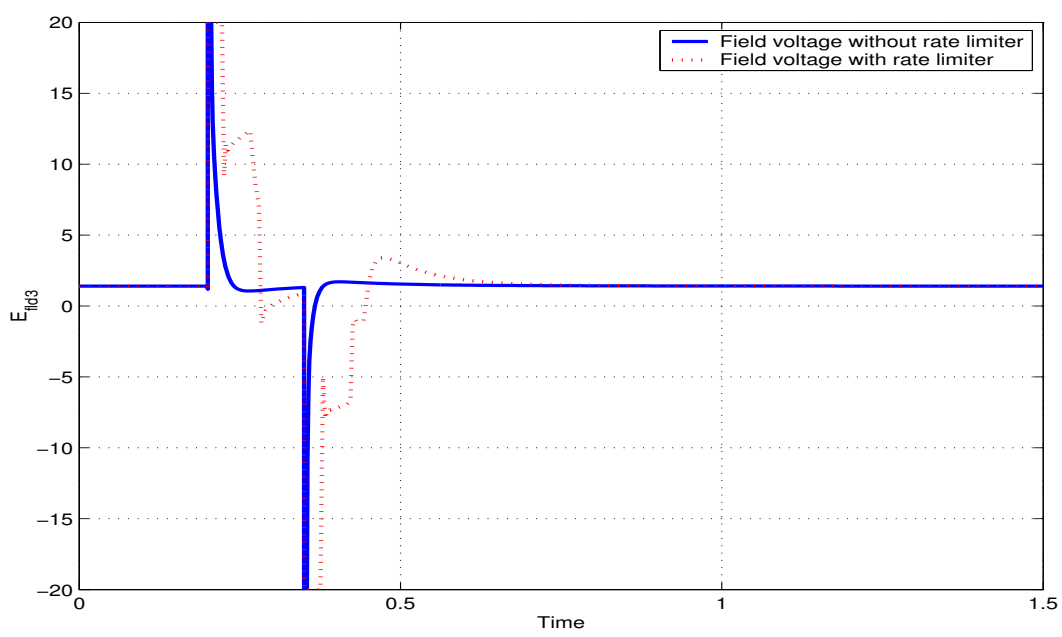

Figure 4.38: Effect of saturation block on third controller signal 


\section{Chapter 5}

\section{Summary and Conclusion}

\subsection{Conclusion and future work}

The nonlinear feedback controller with exact feedback linearization method has been presented for the system of forced Duffing oscillator. The spline method has been explained and computation of piecewise polynomial interpolation for cubic spline concluded. Spline method is used to mimic the EFL controller. Controller effort for Spline-based with different polynomial order, showed better performance in time domain when compared to the EFL. In frequency domain, average of power spectral density of the controller signal is compared with exact feedback linearizaton. Spline-based controller has an advantage of selecting the polynomial order within specific range while in some cases decreasing the controller effort. The degree of piecewise interpolation can be chosen as linear, quadratic, cubic and also higher order and it can be changed easily. It would be interesting to see the application of spline approximation in more general class of nonlinear systems such as Lorenz system.

Second case considered a methodology to control a nonlinear electric power system through the excitation systems. Direct Feedback Linearization technique is used to cancel the nonlinearities of the system and stabilize the system with classical methods such as Linear Quadratic Regulator and genetic algorithms. Cubic spline technique is applied to approximate the complicated nonlinear controller with piecewise polynomial construction. Performance of two controllers are compared for three machine nine bus test system. The system performance under different contingencies is tested. Simulation results show that the proposed controller can enhance the transient stability regardless of different types of faults. 
DFL controller requires unmeasurable internal variables. Internal variable identifier block is presented in order to construct the unmeasurable variables for two axis model. DFL method has two stages for designing the controller. First, nonlinear compensator is designed in a way that it can explicitly cancel the nonlinearities present in the synchronous machine. Second, linear compensator is obtained to achieve particular control objective which stabilize the overall system. Nonlinear simulation demonstrates the effectiveness of the approach in enhancing the stability of the system.

For multi-machine power system, transient stability simulation is the most important tool for planning, design, and operation. Models of generator in power system are important factors in power system. Two types of generator with transient model are mentioned. Controller designed with DFL method is obtained separately for each. Even though DFL controller for two-axis model is complex but it shows more detailed characteristic and dynamics from the system which are relevant to more sophisticated and complete controller design.

Comparison needs to be done between DFL excitation system and standard exciter models. Standard exciter models such as excitation system with dc exciter, static excitation systems with controlled rectifiers, and the excitation systems with ac exciters plus rectifiers are all classified as classical exciters. The proposed algorithm based on spline approximation was tested on 3machine 9-bus test system. It would be more interesting to see the effect of spline controller on larger size system with more detail in generator modelings such as sub-transient modelling.

The main objective of designing the controller with DFL technique and later with spline based is improvement in stability enhancement. Voltage regulation is also another factor that can be considered in controller design procedure. So, the controller needs to be designed in a way that can satisfy transient stability and voltage regulation at the same time. Finally, it should be pointed out that the direct feedback linearization technique is a general method and it can be applied to more detailed excitation system. In fact, the further modelling could include excitation dynamics, voltage regulation, stabilizer and excitation limits. 


\section{Appendix A}

\section{One and Two-Axis Generator Models}

In this appendix, a complete model for synchronous machines with one-axis and two-axis models are considered. A mathematical model for the machine includes differential and algebraic equations. In two-axis generator model, a synchronous generator is represented a voltage source behind transient reactance. Machine dynamics for the two-axis model of the $i^{t h}$ machine with excitation $E_{f l d i}$ are given with following differential equations

$$
\begin{aligned}
\dot{\delta}_{i}(t) & =\omega_{i}(t)-\omega_{0} \\
\dot{\omega}_{i}(t) & =-\frac{D_{i}}{2 H_{i}}\left(\omega_{i}(t)-\omega_{0}\right)+\frac{\omega_{0}}{2 H_{i}}\left(P_{m i}-P_{e i}\right) \\
\dot{E}_{d i}^{\prime} & =\frac{1}{T_{q o i}^{\prime}}\left[I_{q i}\left(X_{q i}-X_{q i}^{\prime}\right)-E_{d i}^{\prime}\right] \\
\dot{E}_{q i}^{\prime} & =\frac{1}{T_{d o i}^{\prime}}\left[E_{f l d i}-I_{d i}\left(X_{d i}-X_{d i}^{\prime}\right)\right]
\end{aligned}
$$

Swing equation as shown in equation number A.2 is important equation for synchronous generator dynamics. First swing stability criteria based on the fact that the generator will not loose its synchronism for the first swing of the rotor angle. The equation shows the dynamic relation between motion of rotor in accordance with Newton's second law. It also shows important characteristic of synchronism for rotor angles. 
Algebraic equations for two axis model are given as

$$
\begin{aligned}
P_{e i} & =E_{d i}^{\prime} I_{d i}+E_{q i}^{\prime} I_{q i}+\left(X_{q i}^{\prime}-X_{d i}^{\prime}\right) I_{d i} I_{q i} \\
E_{d i} & =E_{d i}^{\prime}-I_{d i} r_{a i}+I_{q i} X_{d i}^{\prime} \\
E_{q i} & =E_{q i}^{\prime}-I_{q i} r_{a i}+I_{d i} X_{d i}^{\prime} \\
I_{q i}(t) & =\sum_{j=1}^{n} E_{q j}^{\prime}\left(B_{i j} \sin \delta_{i j}(t)+G_{i j} \cos \delta_{i j}(t)\right) \\
I_{d i}(t) & =\sum_{j=1}^{n} E_{q j}^{\prime}\left(G_{i j} \sin \delta_{i j}(t)-B_{i j} \cos \delta_{i j}(t)\right)
\end{aligned}
$$

The model is converted to simpler two axis that considered in this study by ignoring transient saliency by assuming $X_{q i}^{\prime}=X_{d i}^{\prime}$. Definition for parameters are given in Table A.1

Table A.1: Parameters for two axis model

\begin{tabular}{|c|l|}
\hline$\delta_{i}$ & Rotor angle in radians \\
\hline$\omega_{i}$ & Relative speed in radians per second \\
\hline$P_{m i}$ & Mechanical input power in per unit \\
\hline$P_{e i}$ & Electrical power in per unit \\
\hline$H_{i}$ & Per unit inertia constant in seconds \\
\hline$D_{i}$ & Damping constant in per unit \\
\hline$T_{d o i}^{\prime}$ & Direct axis open circuit time constant in seconds \\
\hline$T_{q o i}^{\prime}$ & Quadrature axis open circuit time constant in seconds \\
\hline$I_{d i}$ & Direct axis current \\
\hline$I_{q i}$ & Quadrature axis current in per unit \\
\hline$E_{d i}^{\prime}$ & Transient EMF in direct axis in per unit \\
\hline$E_{q i}^{\prime}$ & Transient EMF in quadrature axis in per unit \\
\hline$E_{d i}$ & EMF in direct axis, in per unit \\
\hline$E_{q i}$ & EMF in quadraturee axis, in per unit \\
\hline$E_{f l d i}$ & Equivalent EMF in excitation coil in per unit \\
\hline$X_{d i}^{\prime}$ & Transient reactance in direct axis \\
\hline$X_{q i}^{\prime}$ & Transient reactance in quadrature axis \\
\hline
\end{tabular}

The one axis generator is similar to two-axis model except the dynamics of $E_{d i}^{\prime}$ is ignored. So the differential equations are the same except for equation A.3.

Differential equations are 


$$
\begin{aligned}
\dot{\delta}_{i}(t) & =\omega_{i}(t)-\omega_{0} \\
\dot{\omega}_{i}(t) & =-\frac{D_{i}}{2 H_{i}}\left(\omega_{i}(t)-\omega_{0}\right)+\frac{\omega_{0}}{2 H_{i}}\left(P_{m i}-P_{e i}\right) \\
\dot{E}_{q i}^{\prime}(t) & =\frac{1}{T_{d o i}^{\prime}}\left(E_{f l d i}(t)-E_{q i}(t)\right)
\end{aligned}
$$

The algebraic equations are

$$
\begin{aligned}
& E_{q i}(t)=E_{q i}^{\prime}(t)-\left(X_{d i}-X_{d i}^{\prime}\right) I_{d i}(t) \\
& E_{f i}(t)=k_{c i} u_{f i}(t) \\
& P_{e i}(t)=E_{q i}^{\prime} I_{q i}+\left(X_{q i}-X_{d i}^{\prime}\right) I_{q i} I_{d i} \\
& Q_{e i}(t)=E_{q i}^{\prime}(t) I_{d i}(t) \\
& I_{q i}(t)=\sum_{j=1}^{n} E_{q j}^{\prime}\left(B_{i j} \sin \delta_{i j}(t)+G_{i j} \cos \delta_{i j}(t)\right) \\
& I_{d i}(t)=\sum_{j=1}^{n} E_{q j}^{\prime}\left(G_{i j} \sin \delta_{i j}(t)-B_{i j} \cos \delta_{i j}(t)\right)
\end{aligned}
$$

Parameters are given in Table A.2

Table A.2: Parameters for one axis model

\begin{tabular}{|l|l|}
\hline$k_{c i}(t)$ & The gain of excitation amplifier, in per unit \\
\hline$u_{f i}(t)$ & The input of amplifier, in per unit \\
\hline$P_{e i}(t)$ & Active power in per unit \\
\hline$Q_{e i}(t)$ & Reactive power in per unit \\
\hline$I_{q i}(t)$ & Quadrature axis current in per unit \\
\hline$I_{d i}(t)$ & Direct axis current in per unit \\
\hline$E_{q i}^{\prime}(t)$ & Transient EMF in quadrature axis in per unit \\
\hline$E_{q i}(t)$ & EMF in quadrature axis, in per unit \\
\hline
\end{tabular}




\section{References}

[1] Y. L. Abdel-Magid, M. Bettayeb, and S. Z. Selim, Shokri, "Genetic Algorithm Based Simultaneous Eigenvalue Placement of Power Systems," Arabian Journal for Science and Engineering, Section C: THEME ISSUES, Optimization Theory and Applications, Vol. 25, Number 1C, June 2000, pp. 63-78.

[2] P. M. Anderson and A.A Fouad, Power System Control and Stability, IEEE Press, New York, 1994.

[3] C. de Boor and The MathWorks, Inc. Spline Toolbox For Use With Matlab, Version 2, January 1999.

[4] C. de Boor, A practical guide to splines Revised Edition, Springer-Verlag 2001.

[5] R. L. Burden, and D. Faires, Numerical Analysis, PWS Publishing Company, 1992.

[6] G. Chen, X. Dong, "From Chaos to order: Perspectives and Methodologies in controlling chaotic nonlinear dynamical systems," International Journal of Bifurcation and Chaos, Vol. 3, 1993, pp. 1363-1409.

[7] L. Q. Chen and Y. Z. Liu, "A Modified Exact Linearization Control For Chaotic Oscillators,", Nonlinear Dynamics Vol. 20, 1999, pp. 309.

[8] A. Chipperfield, P. Fleming, H. Pohlheim, C. Fonseca, Genetic Algorithm Toolbox For Use With Matlab: User's Guide, Version 1.2, Department of Automatic Control and Systems Engineering, University of Sheffield, 2001.

[9] X. Dong, G. Chen, "Controlling Chaotic Continous-Time Systems via Feedback," Proceedings of the 31st IEEE Conference on Decision and Control, Westin La Paloma, Tucson, Arizona USA, December 1992.

[10] X. Dong, G. Chen, L. Chen, "Controlling the Uncertain Duffing Oscillator," Proc.1st Int. Conf. on Control of Oscillations and Chaos, Vol. 3, 1997, pp. 419-422.

[11] Z.Y. Dong, Y.V. Makarov and David J. Hill, "Power System Small Signal Stability Analysis Using Genetic Optimization," International Journal of Electric Power Systems Research, September 1998. 
[12] S. A. Dyer, J. S. Dyer, "Cubic-spline Interpolation: Part 1," IEEE Trans. on Instrumentation and Measurement, Vol. 4, Issue 2, March 2001, pp. 44-46.

[13] S. A. Dyer, H. Xin, "Cubic-Spline Interpolation: Part 2," IEEE Trans. on Instrumentation and Measurement, Vol. 4, Issue 2, June 2001, pp. 34-36.

[14] C. F. Gerald, and P. O. Wheatly, Applied Numerical Analysis, Menlo Park, California, Addison Wesley, 1984, pp. 579.

[15] T. Glad, L. Ljung, Control Theory, Taylor and Francis, New York, 2000.

[16] Y. Guo, D. J. Hill, Y. Wang, "Nonlinear Decentralized Control of Large-scale PowerSystems," IEEE Trans. on Automatica, Vol. 36, 2000, pp. 1275-1289.

[17] A. Isidori, Nonlinear Control Systems, Springer-Verlag, New York, 1989.

[18] Z. P. Jiang, "New Results on the Feedback Control of Duffing's Equation," Proceedins of the 40th IEEE, Conference on Decision and Control, Orlando, Florida USA, December 2001.

[19] A. Karimi, A. Feliachi, "Spline-based nonlinear controller for the Duffing oscillator ," Proceedings of the 35th Southeastern Symposium on System Theory, March 2003, pp. 191-195.

[20] A. Karimi, A. Feliachi, "Spline-based controller for power system stability enhancement," 2003 Large Engineering Systems Conference on Power Engineering, May 2003, pp. 90-94.

[21] H. K. Khalil, Nonlinear Systems, Macmillan Publishing Company, New York, 1992.

[22] E. Kyriakides, G. T. Heydt, "An Observer for the Estimation of Synchronous Generator Damper Currents for Use in Parameters Identification," IEEE Trans. on Energy Conversion, Vol. 18, No. 1, March 2003, pp. 175-177.

[23] A. A. J. Lefeber, H. Nijmeijer, "Bounded tracking controllers for the chaotic(forced) Duffing equation," Proc.1st Int. Conf. on Control of Oscillations and Chaos, Vol.3, 1997, pp. 415418.

[24] J. Machowski, J. Bialek, J. R. Bumby, Power System Dynamics and Stability, WILEY CANADA, 1999.

[25] A. N. Netravali and R. J. P. "On a Class of Minimum Energy Controls Related to Spline Functions," Automatic Control, Vol. 21, October 1976, pp. 725-727.

[26] P.W. Sauer and M.A. Pai, Power System Dynamics and Stability, Prentice-Hall, 1998.

[27] G. M. Siouris, Optimal Control and Estimation Theory, John Wiley and Sons, New York, 1996.

[28] D. G. Taylor, Nonlinear control of electric machines: an overview , Control Systems Magazin IEEE, Vol. 14, Dec. 1994.

[29] M. Vidyasagar, Nonlinear Systems Analysis, Prentice Hall, Englewood Cliffs, New Jersey 07632,1993 . 
[30] Y. Wang, G. Guo, D. J. Hill and L. Gao, "Nonlinear Decentrilized Control For Multimachine Power System Transient Stability Enhancement," Proceedings of Stockholm Power Tech International Symposium on Electric Power Engineering, 1995, pp. 435-440.

[31] Y. Wang, D. J. Hill, L. Gao and R. H. Middleton, "Transient Stability Enhancement and Voltage Regulation of Power System," IEEE Trans. on Power systems, PWRS-8, 1993, pp. 620-627.

[32] T. H. Yeap, N. U. Ahmed, "Feedback Control of Chaotic System," International Journal of Dynamics and Control, Vol. 4, 1990, pp. 97-110.

[33] C. Zhu, R. Zhou, Y. Wang, "A New Decentralized Nonlinear Voltage Controller For Multimachine Power Systems," IEEE Transaction on Power Systems, Vol. 13, No. 1, February 1998, pp. 211-216. 